2018-07-10

\title{
Identifying the Optimum Zone for Reducing Drill String Vibrations
}

\author{
Etaje, Darlington Christian
}

Etaje, D. C. (2018). Identifying the Optimum Zone for Reducing Drill String Vibrations (Unpublished master's thesis). University of Calgary, Calgary, AB. doi:10.11575/PRISM/32358 http://hdl.handle.net/1880/107136

Downloaded from PRISM Repository, University of Calgary 


\title{
UNIVERSITY OF CALGARY
}

Identifying the Optimum Zone for Reducing Drill String Vibrations

by

Darlington Christian Etaje

\author{
A THESIS \\ SUBMITTED TO THE FACULTY OF GRADUATE STUDIES \\ IN PARTIAL FULFILMENT OF THE REQUIREMENTS FOR THE \\ DEGREE OF MASTER OF SCIENCE
}

GRADUATE PROGRAM IN CHEMICAL ENGINEERING

CALGARY, ALBERTA

JULY, 2018

(C) Darlington Christian Etaje 2018 


\begin{abstract}
This thesis was written to address the vibration problems that occur during drilling operations. Due to the rotational motion effected on the drill string while drilling, vibrations occur, and when these vibrations become excessive, the drill string may oscillate in a manner that could damage the pipes and damage other tools attached to the drill string. Machine learning may be used to identify the vibration prone zones and provide recommendations to the driller to change the operating weight on bit (WOB) and rotation speed (RPM) to achieve drilling efficiency while reducing the possibility of damages downhole. Data received from the rig is processed through a dimension reduction process and then categorized using a decision tree classification method. The rules behind the decision tree was created by reversing conventional ways of curbing vibration problems during drilling operations.

In the course of the research, it was discovered that there is a need for additional safety gap away from the usual boundary for vibration problems. Quantitative risk analysis was used to identify this gap. This report explains the process of identifying that safety gap. The machine learning model used throughout this research was trained on recorded downhole data and tested with surface data from the electronic drilling recorder.

The reports also highlight the findings from market research done to identify the possibility of deploying this research as a startup in Calgary, Canada. Detailed competitor analysis is shown based on customer discovery and customer validation interviews. This has led to the development of business model canvas which is described in this report. A blue ocean strategy was graphed showing that the startup, "Optimum Zone Identifier, OZI" can be differentiated from competitors by being in a market segment that has unique needs with OZI being the only player fitting this category.
\end{abstract}




\section{Acknowledgements}

I would like to specially thank my supervisor, Professor Roman Shor, for giving me the opportunity and funding to work on this project and for believing in me. My sincere appreciation also goes to Chemical and Petroleum Engineering Department for providing research resources and opportunities for broader experience in terms of pursuing a path to entrepreneurial success.

I would also like to express my profound gratitude and appreciation to Hunter Centre for Entrepreneurship and Innovation, Innovate Calgary, and Faculty of Graduate Studies for granting me the opportunity guiding me through customer discovery and customer validation interviews which ensured I got a better view of the industry perspective of the usefulness of my project. 


\section{Dedication}

I would like to dedicate this work to my supervisor, my parents, my wife, my wife's family, my church TREM Calgary and my daughter for all their prayers and emotional support. 


\section{Table of Contents}

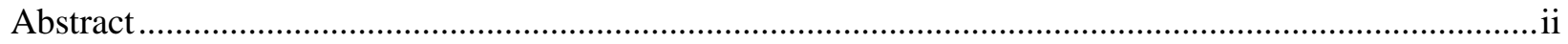

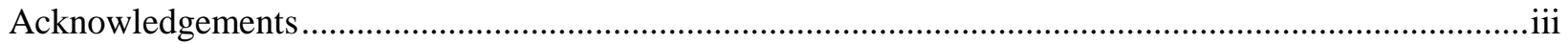

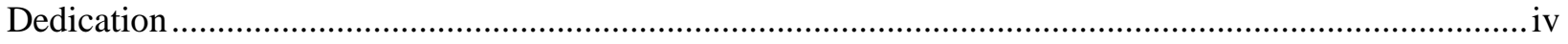

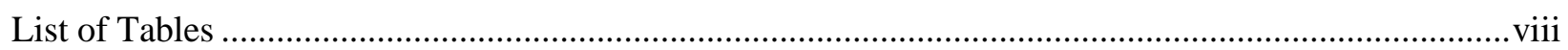

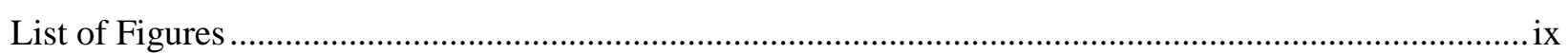

List of Symbols, Abbreviations and Nomenclature ...........................................................................

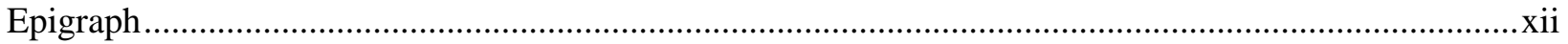

Chapter One: INTRODUCTION TO DRILL STRING VIBRATION ................................................ 1

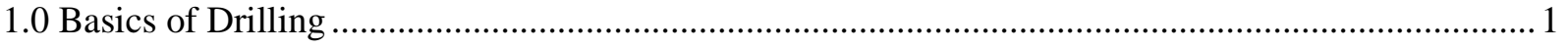

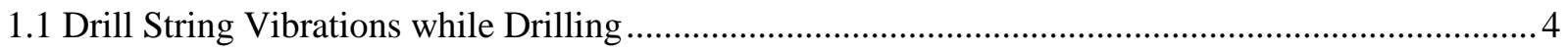

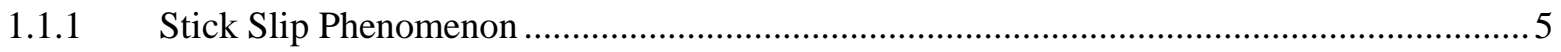

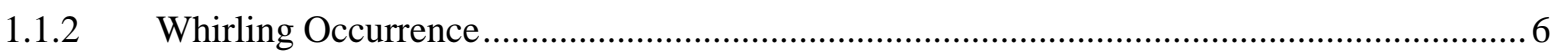

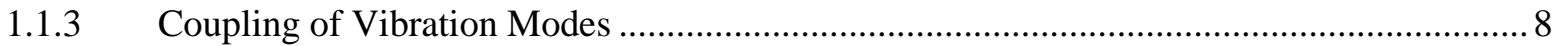

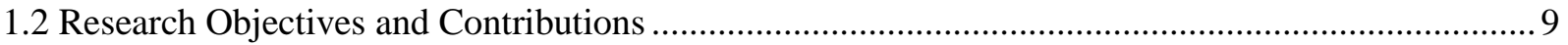

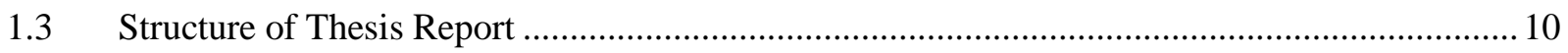

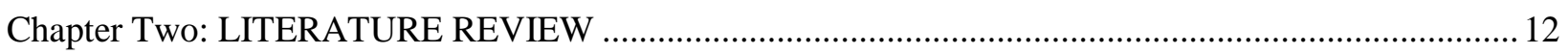

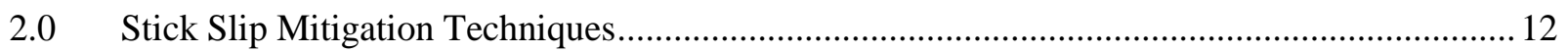

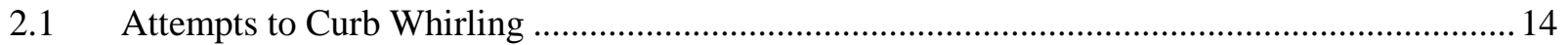

2.2 Overview of Technologies for Mitigating Drill String Vibrations ........................................ 15

2.3 Software for Identifying Vibration Problems ....................................................................... 16

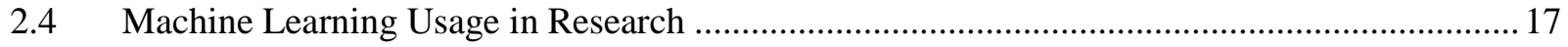

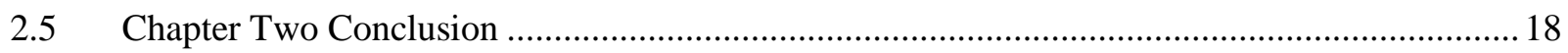

Chapter Three: USING MACHINE LEARNING TO IDENTIFY VIBRATION .................................. 19

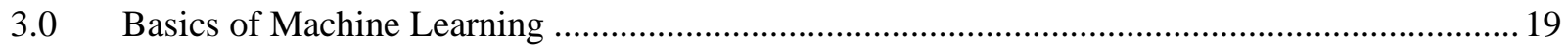

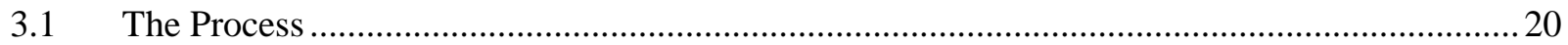

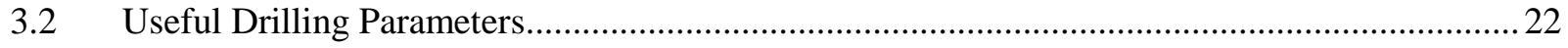

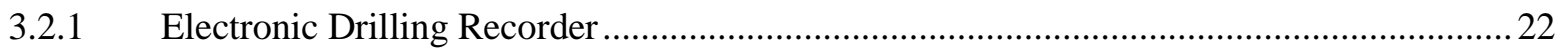

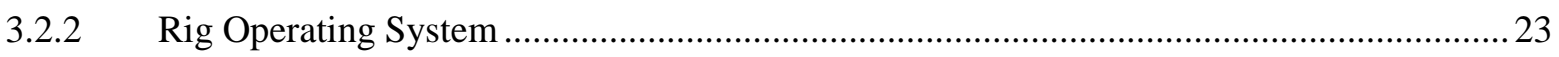

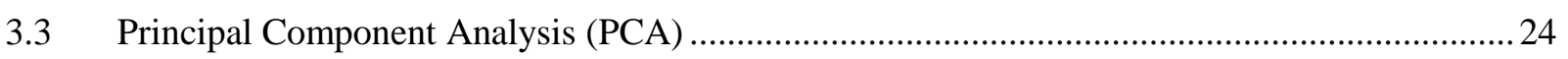

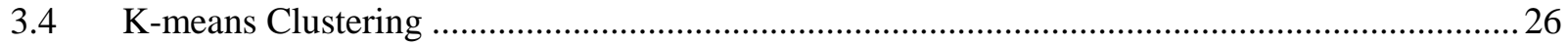

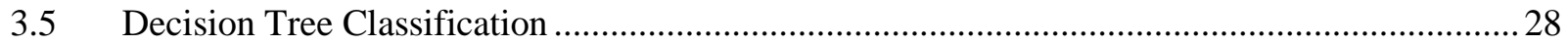

3.6 Chapter Three Conclusion ……..................................................................................... 30

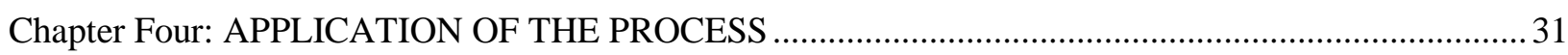

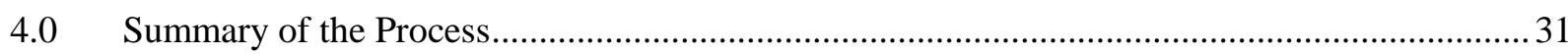

4.1 Convention Operational Techniques for Curbing Vibration................................................... 31

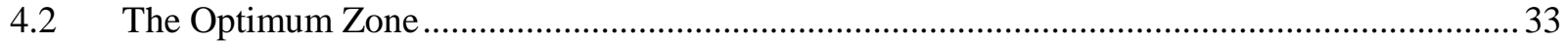




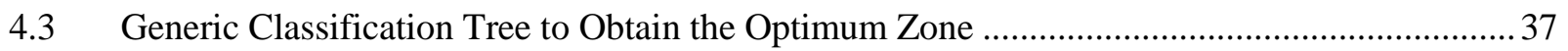

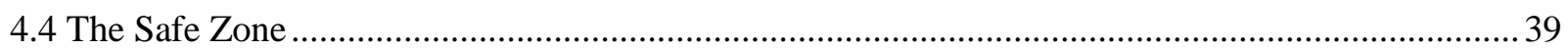

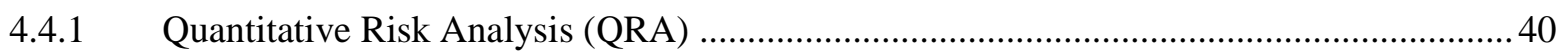

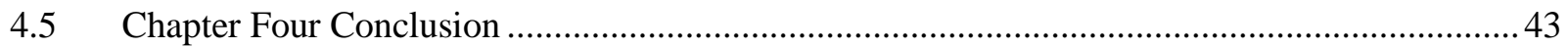

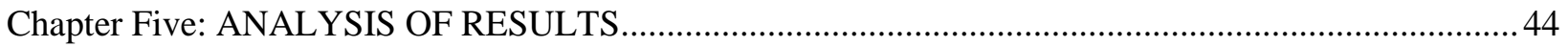

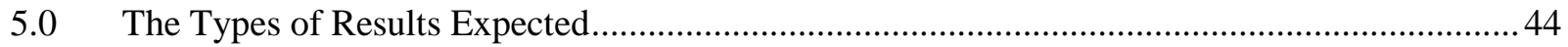

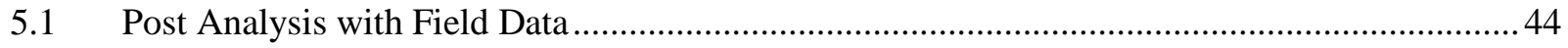

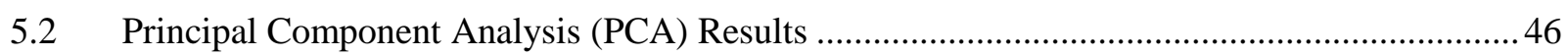

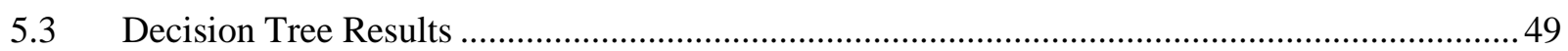

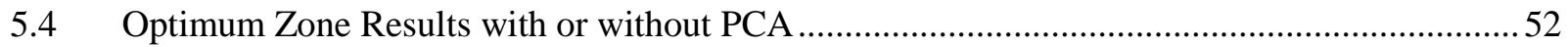

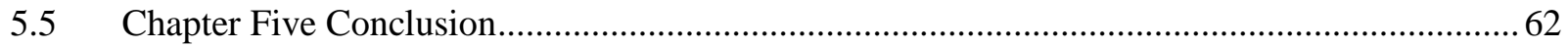

Chapter Six: BUSINESS OPPORTUNITIES FOR OPTIMUM ZONE RESEARCH...........................63

6.0 Basics of Customer Discovery and Customer Validation..........................................................63

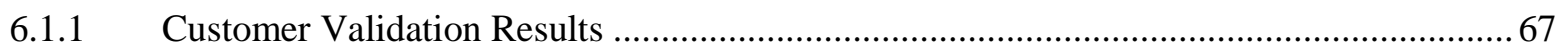

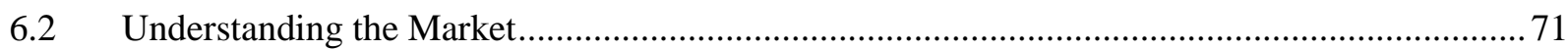

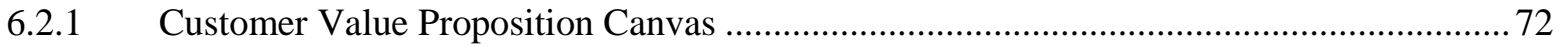

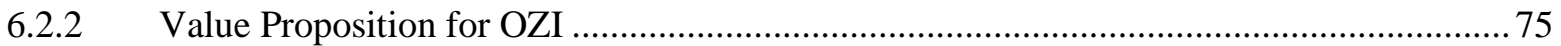

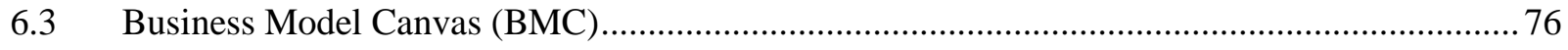

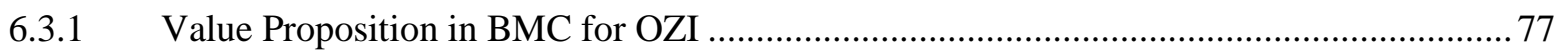

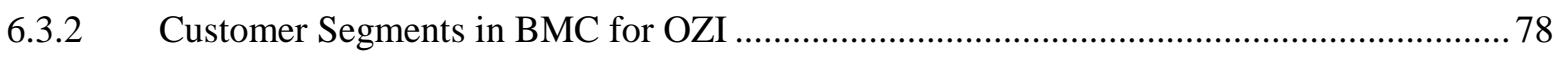

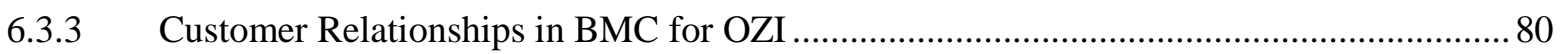

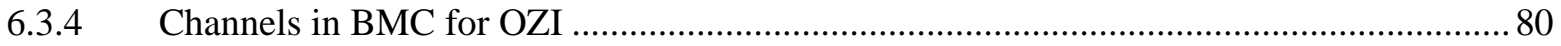

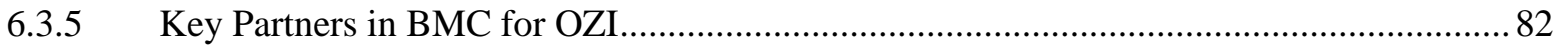

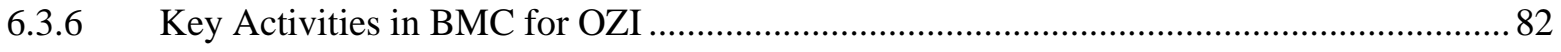

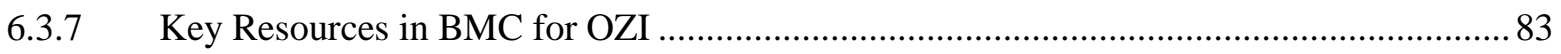

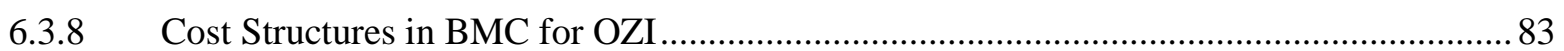

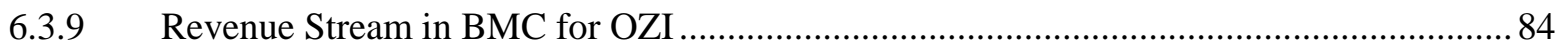

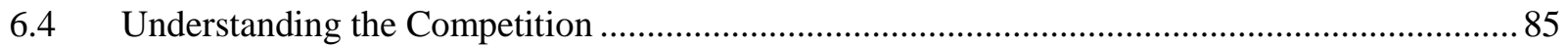

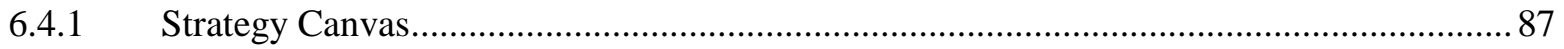

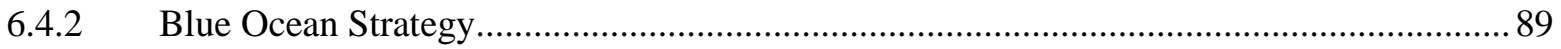

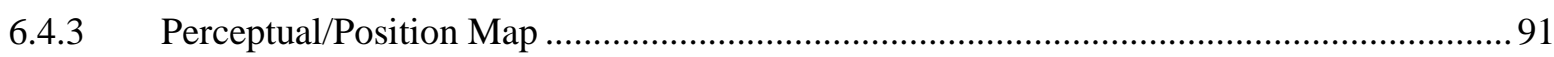

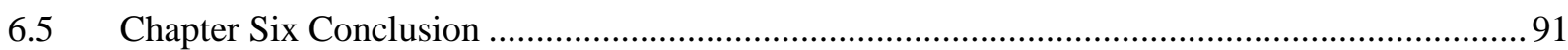

Chapter Seven: CONCLUSIONS AND RECOMMENDATIONS …................................................ 92

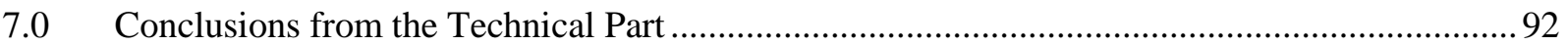

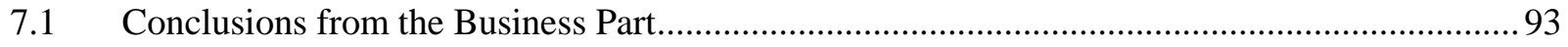

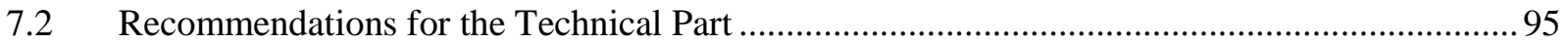




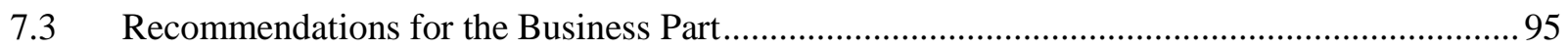

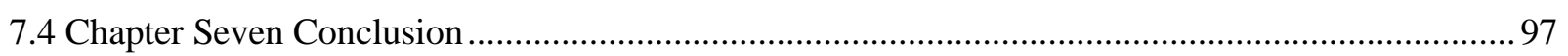

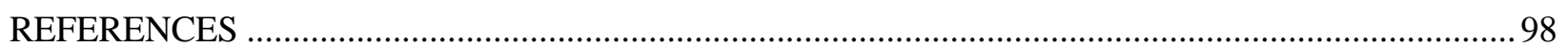

APPENDIX I: Matlab Code for Optimum Zone/Safe Zone Chart ....................................................... 105

APPENDIX II: CUSTOMER VALUE PROPOSITION CANVAS WITH REFERENCES .................... 110 


\section{List of Tables}

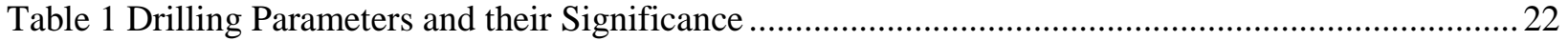

Table 2 Types of Nodes in a Decision Tree and their Characteristics ...................................................22

Table 3 Significance Level for Principal Components in Update One ...................................................46

Table 4 Significance Level for Principal Components for Updates One to Update Seven........................47

Table 5 Vibration Severity Levels Based on Downhole Measurements..................................................53

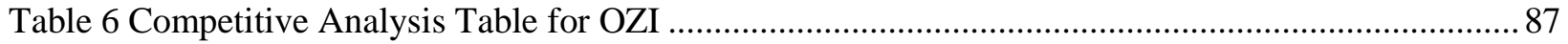




\section{List of Figures}

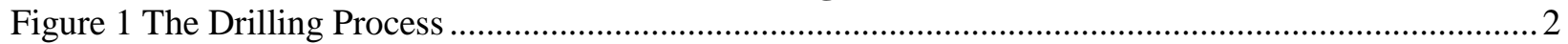

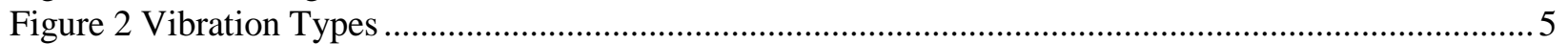

Figure 3 Effect of Stick/Slip on Downhole RPM ........................................................................... 6

Figure 4 Effect of Whirling on Downhole RPM.............................................................................. 7

Figure 5 Forward and Backward Whirling ……………..............................................................

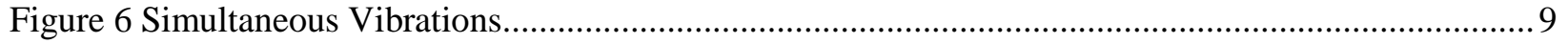

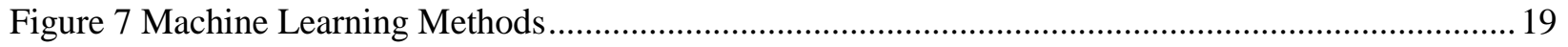

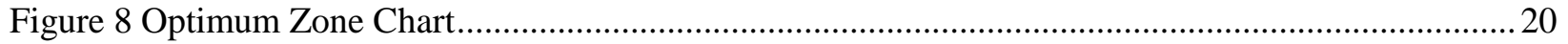

Figure 9 Using Machine Learning to Identify Optimum Zone and Safe Zone ..........................................2.

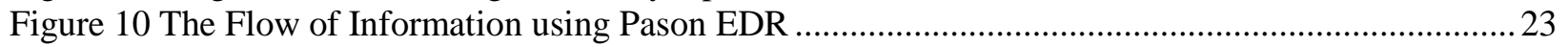

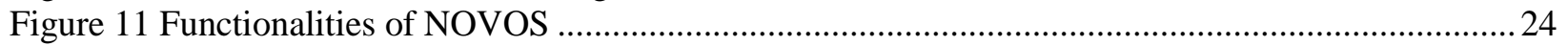

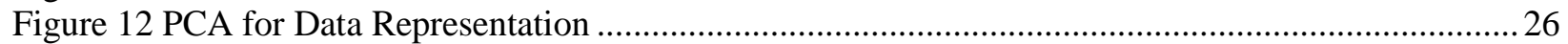

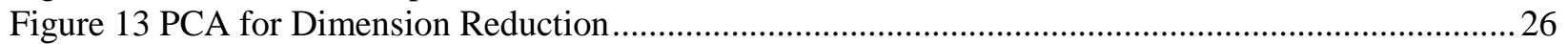

Figure 14 Elbow Point Method for Determining the Value of K............................................................22

Figure 15 Decision Tree for Mammal Classification ………………………………………………....2

Figure 16 Drilling Optimization Mitigation Flow ……………........................................................... 32

Figure 17 Conventional Ways of Curbing Lateral and Torsional Vibrations ...............................................3

Figure 18 Illustration of Eigenvectors on a PCA Chart ……………………………………………....... 35

Figure 19 Obtaining Upper and Lower Limits of WOB and RPM .........................................................36

Figure 20 Basic Optimum Zone Chart............................................................................................. 37

Figure 21 Decision Tree Classification to Obtain Optimum Zone Chart for Reducing Drill String

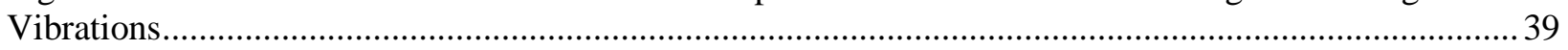

Figure 22 Optimum Zone Chart Showing Safe Zone ............................................................................ 42

Figure 23 Depth Versus Time for the Well used for Post Analysis............................................................4

Figure 24 Identifying the Stand for the Post Well Analysis ....................................................................46

Figure 25 3D Plot of 1st, 2nd and 3rd Principal Components for Update 1 ..........................................4

Figure 26 3D Plot of 1st, 2nd and 3rd Principal Components for Update 2 and Update 3 .........................48

Figure 27 3D Plot of 1st, 2nd and 3rd Principal Components for Update 4 and Update 5 .........................48

Figure 28 3D Plot of 1st, 2nd and 3rd Principal Components for Update 6 and Update 7 ........................49

Figure 29 WOB versus RPM Plot on Matlab Classification Learner App ………………………….......50

Figure 30 Decision Tree Result from Random Well Data ........................................................................51

Figure 31 Revised Generic Decision Tree to Obtain Optimum Zone.........................................................52

Figure 32 Classification Based on Downhole Data for Update 1 .............................................................54

Figure 33 Classification with PCA and Safety for Update 1 ………….................................................5

Figure 34 Classification Based on Downhole Data for Update 2 ..............................................................5

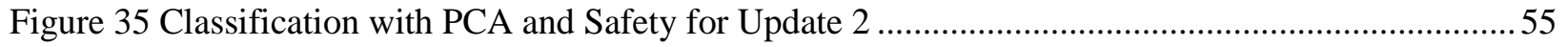

Figure 36 Classification Based on Downhole Data for Update 3 .............................................................56

Figure 37 Classification with PCA and Safety for Update 3 ………………………………………......56

Figure 38 Classification Based on Downhole Data for Update 4 ……………………………………......57

Figure 39 Classification with PCA and Safety for Update 4 ……….....................................................5

Figure 40 Classification Based on Downhole Data for Update 5 ……………………………………......58

Figure 41 Classification with PCA and Safety for Update 5 ………………………...........................5

Figure 42 Classification Based on Downhole Data for Update 6 ……………………………..................59

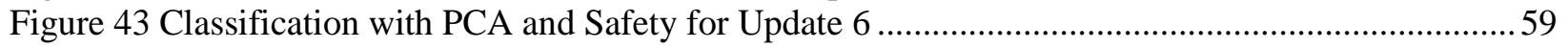

Figure 44 Classification Based on Downhole Data for Update 7 ..............................................................6

Figure 45 Classification with PCA and Safety for Update 7 ………………………...........................6

Figure 46 Classification Based on Downhole Data for Good Drilling Stand .............................................61

Figure 47 Classification with PCA and Safety for Good Drilling Stand ..................................................6 61

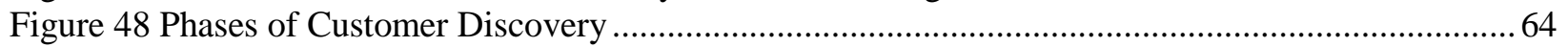

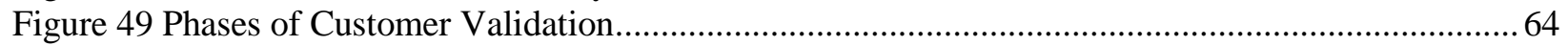


Figure 50 Pricing Model Birth from Customer Validation Interviews Summer 2017............................67

Figure 51 NRGX Technologies Company Structure based Customer Validation Interview..................... 68

Figure 52 A Typical Adoption Life Cycle for New Technologies .........................................................69

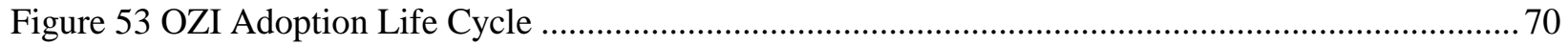

Figure 54 Equating Business Model Canvas with Value Proposition Canvas........................................... 72

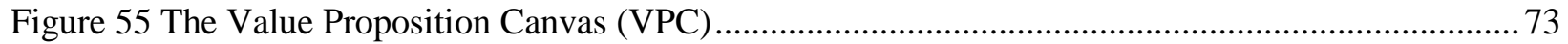

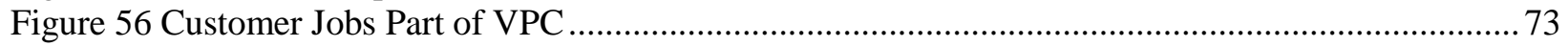

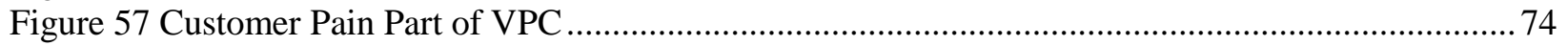

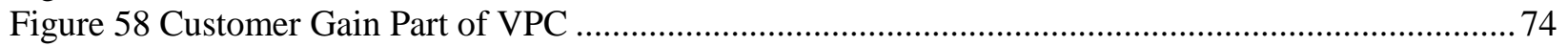

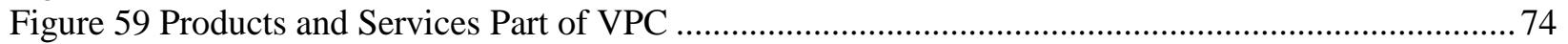

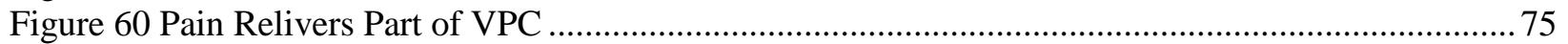

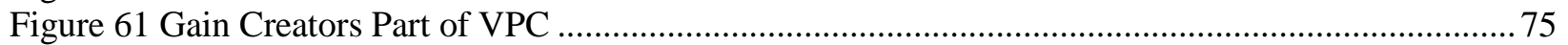

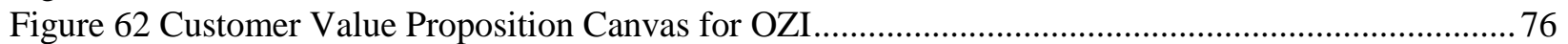

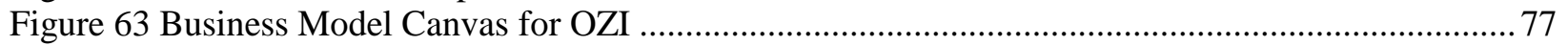

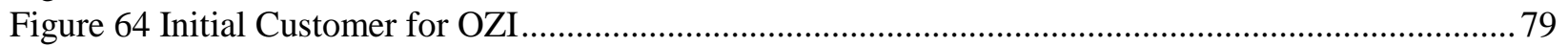

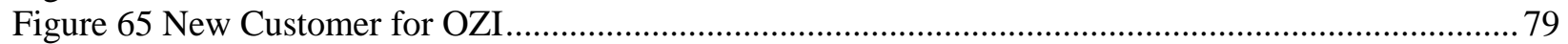

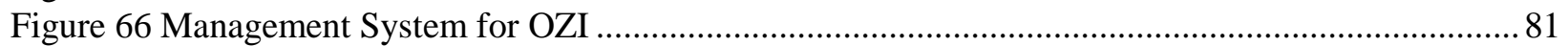

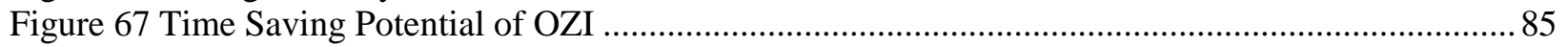

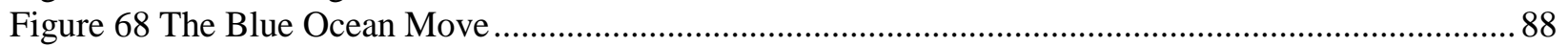

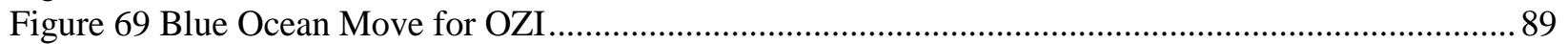

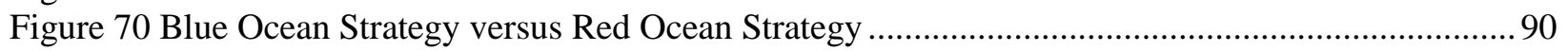

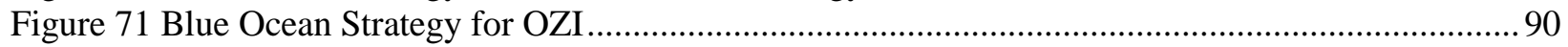

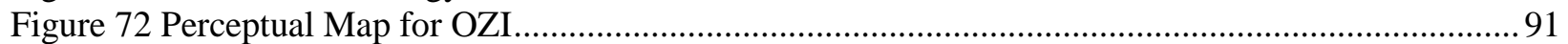

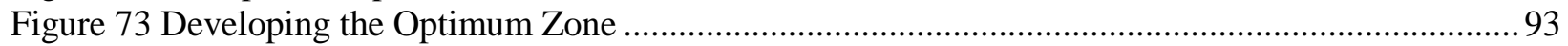

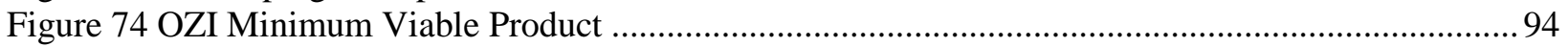

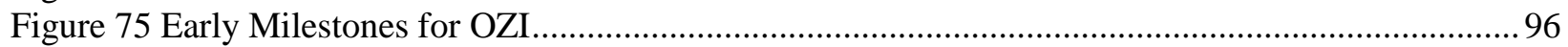




\section{List of Symbols, Abbreviations and Nomenclature}

Symbol

WOB

RPM

ROP

TOR

OZI

BOP

TD

MSE

MWD

LWD

PDC

DS

BHA

NOV

NOVOS

3D

NAFLV

FEA

SAX

NPT

EOR

d

PCA

EDR

WITS

$\mathrm{K}$

$\mathrm{BD}$

DMEA

SIP

TALC

BMC

MVP

NRGX

VPC
Definition

Weight on Bit (klbf)

Revolutions per Minute

Rate of Penetration (ft/s)

Torque

Optimum Zone Identifier

Blowout Preventer

Target Depth (ft)

Mechanical Specific Energy

Measurement while Drilling

Logging while Drilling

Polycrystalline Diamond Compact

Drill String

Bottom Hole Assembly

National-Oilwell Varco

National-Oilwell Varco Operating System

3 Dimensional

Natural Angular Frequencies of Lateral Vibration

Finite Element Analysis

Symbolic Aggregate Approximation

Non Productive Time

Enhanced Oil Recovery

D-Exponent

Principal Component Analysis

Electronic Drilling Recorder

Wellsite Information Transfer Specification

Number of Clusters

Bit Depth

Measured Depth

Summer Incubator Program

Technology Adoption Life Cycle

Business Model Canvas

Minimum Viable Product

Privately-held company headquartered in Calgary 


\section{Epigraph}

Without change there is no innovation, creativity, or incentive for improvement. Those who initiate change will have a better opportunity to manage the change that is inevitable. William Pollard 


\section{Chapter One: INTRODUCTION TO DRILL STRING VIBRATION}

A basic introduction into vibrations of the drill string is highlighted in this chapter. The different types of vibrations that could occur and their mechanisms are discussed. The scenario where coupling could happen is also explained. The objectives of the research are itemized and their contributions to the drilling industry are listed. The entire project report is then summarized on a per chapter basis.

\subsection{Basics of Drilling}

In order to extract the natural oil and gas resources in the earth's subsurface, there is a need to drill through the ground to a depth where these natural resources can be found. Usually, this involves a study of the subsurface (or formation) till a good understanding of the geology of the target area is revealed. A quality geological and geophysical study is done; it is on this basis that the drilling site is chosen and preparations for drilling kicks off [2]. An overview of the drilling process for a land rig is shown in Figure 1 below. After the location is chosen, the site is prepared for drilling. The surface hole is drilled and the corresponding casing is cemented in place. The process of drilling, casing, cementing is continued until the target depth is reached. The appropriate stimulation is applied and production equipment are installed to produce oil or gas from the reservoir.

Looking at the entire drilling process on a step-by-step basis; well location would be the first step. The reservoir may be at a subsurface location which may not be favorable for drilling from the corresponding surface location. This means the driller may want to apply directional drilling or horizontal drilling and not just vertical drilling. So many factors affect the choice of the well location: environmental factors, rules from the regulating body, the formation type anticipated, the

formation strength and so on. One major factor that affects the well location is the economics of the target reservoir. Other factors affecting selection of well location are oil and gas lease covering the tract, surface topography, subsurface geology, and reservoir characteristics [77]. 


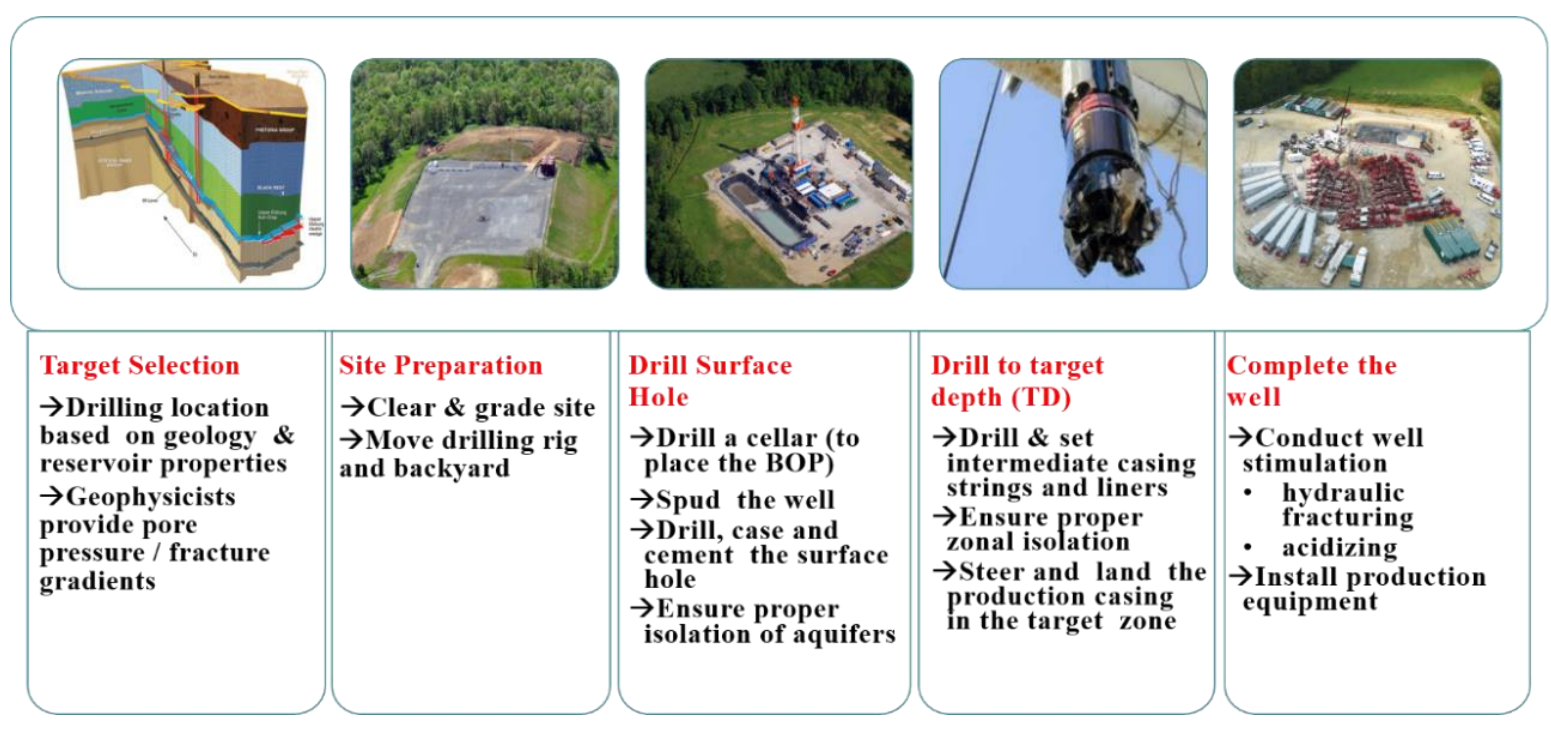

\section{Figure 1 The Drilling Process [2]}

The drilling team would prefer to reach the target zone from a position where the bulk of the reservoir can be produced safely without causing too much damage to the surrounding rocks [2]. This is because the formation already has a rock strength that corresponds with its rock stresses before the drilling starts; there would be a change in equilibrium between the rock strength and the rock stresses when the drilling begins. It is the job of the drilling team to ensure that the borehole stability is maintained during the drilling process so the well does not collapse or fracture. Also, the driller would have to take note of the interaction between the formation fluids and the borehole fluids; controlling the density of the borehole fluids to prevent loss circulation or kick [78].

The well path design is also key to the drilling process. The drilling team has to design the well path in the most advantageous route as much as possible. Often, the actual well path deviates a little from the original design of the well path. This could be due to faults and unpredicted overpressured or underpressured zones. The parameters of importance are the target inclination and azimuth at each stage of the drilling process. The well path design chosen and the anticipated formation to be encountered will inform the drilling team what kind of casing program and cementing design would be necessary for the drilling. The information on the kind of formation expected to be encountered would help with the well design. At this point, the load and capacity 
of each section of the well can be determined. Questions to be answered would be what kind of pressure would cause fracture or breakout of the well. The formation stress would be important as well.

The general idea is that the well must be stable enough so that while drilling fluids do not flow into the formation (a phenomenon called loss circulation) and to prevent unwanted fluids in the formation from flowing into the well up to the surface (a phenomenon called kick). This is where the mud system comes in. The drilling mud is a fluid run through the drill string to exert hydrostatic pressure on the wellbore to match or minimally exceed the bottomhole pressure. Since the well path is known and the casing design has been developed based on the well design, the mud system can be effected as that additional weight to keep the well stable during drilling. This is determined based on the formation pore pressure expected (pressure due to fluids in the pores of the formation). The most important use of the drilling mud would be well control. This birth the concept of the drilling window. The mud density must be in-between the pore pressure and the fracture pressure. If the drilling mud density is lower than the pore pressure gradient, kick will occur. Subsequently, if the drilling mud density is higher than the fracture gradient, the well would be fractured and loss circulation could happen. There are 3 types of drilling mud, oil based mud, water based mud and synthetic based muds. Cost and formation pressure are among many factors that affect the choice of the mud system to be used.

The most important parameters of the drilling process are rotational speed (RPM) and the weight on bit (WOB). The drilling process can be optimized by adjusting these parameters based on models and learning that ensure the drilling efficiency is improved. The driller at the drilling console of the rig controls the values of these parameters during drilling. It has been noticed that there is variation in values of these parameters at the surface and down at the drill bit. This means at every instant, the WOB and RPM at the surface may not necessarily be the same with the WOB and RPM at the bottom of the hole. So many factors could cause this variation. It could simply be 
the time lag generated between when the change is effected and when that effect travels down the hole through the drill string. Another cause of this differences is vibration of the drill string.

\subsection{Drill String Vibrations while Drilling}

In most drilling operations, vibrations downhole will occur. Since it takes lowering and rotation of the drill string to drill the well, a kind of vibration would happen which is useful. Simple vibrations due to rotation of the drill string can help cuttings circulate smoothly back to the surface thereby cleaning the hole. However, when this vibration becomes excessive beyond its regular usefulness, at undesired RPM and WOB, it can cause damages to the drill string. Usually, there are measurement while drilling (MWD) tools and logging while drilling (LWD) tools downhole. When these excessive vibrations occur, these tools would be smashed on the wellbore wall repeatedly, eventually damaging these tools and even damaging the drill bit. These vibrations consequently lead to damage of the downhole steerable motor and alteration of the data received from measuring tools attached to the drill string. The rate of penetration would drop and the drilling process would take longer time. Usually when these tools are damaged, the driller would have to search for the point of damage and most times bring out the entire drill string and replace the damaged tools. In the situation where up to the drill string is broken, the removal process is known as fishing. Sometimes if the bad tools cannot be removed or fished out, the driller would sidetrack and redirect the path of the drilling, somehow still aiming for the target zone.

The financial impact of drill string vibration is significant. It contributes typically 20 percent of nonproductive time (NPT) [84]. NPT makes up an average of 20 percent of the rig time which translate into the cost of drilling a well daily [84]. There are 3 modes of vibration that could occur: torsional vibration, known as stick slip, axial vibration or bit bounce, and lateral vibration which is whirling (or bending vibration). Figure 2 below shows the 3 modes of vibration and their direction of motion. 


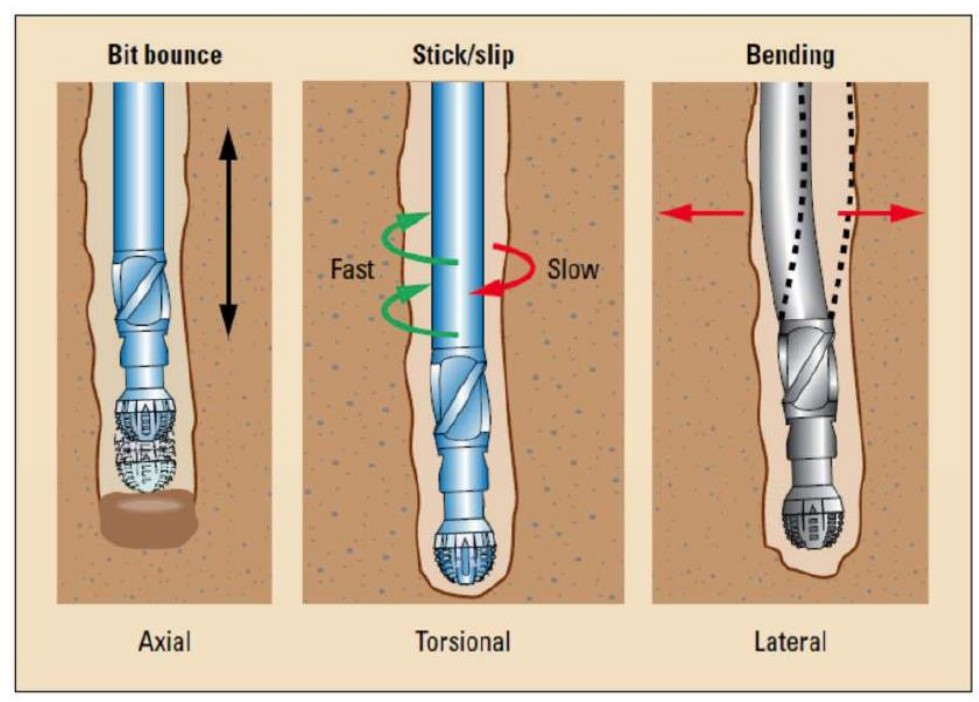

Figure 2 Vibration Types [3]

These 3 modes of vibration are usually studied alone but in reality, they occur simultaneously during drilling. Just by use of polycrystalline diamond compact (PDC) bits, axial vibration can be weakened. That is why this report and research focuses more on combating torsional and lateral vibration.

\subsubsection{Stick Slip Phenomenon}

Stick Slip is a type of torsional vibration with two phases; the sticking phase and the slipping phase. During the sticking phase, the drill string sticks to the wall of the borehole, reducing rotational speed to zero (0 RPM). After a moment, the drill string switches to the slipping phase where the drill string rotates rapidly. The RPM at the surface is constant but the oscillations downhole makes the RPM downhole to be widely different. Figure 3 shows the variation in the RPM at the surface and downhole. The green line represents the desired speed which was fixed at the surface while the blue line shows the deviation from that desired speed which is what was happening downhole. The speed keeps dropping to zero during the sticking phase and increasing to almost 20 times the speed at the surface. 


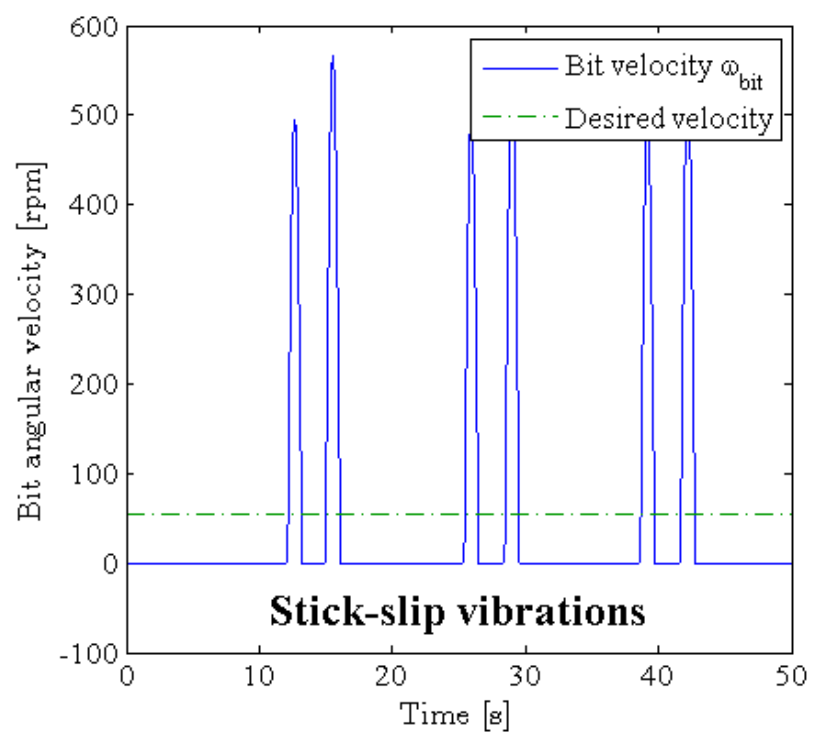

Figure 3 Effect of Stick/Slip on Downhole RPM [6]

Obviously, the tools downhole could get bad and alterations in the mud pulse telemetry are possible. Since the speed is different from the surface speed, the rate of penetration, ROP, could be affected and eventually lowered for harder rocks. That would mean longer drilling time and higher cost of drilling.

\subsubsection{Whirling Occurrence}

The expectation is that the drill string would rotate around its centerline but during lateral vibration (whirling) that is not the case. The drill string revolves around the wall of the wellbore and rotates around its centerline. The phenomenon also causes the rotational speed downhole to be different from the speed at the surface as shown in Figure 4. The broken line represents the surface speed while the full line shows the downhole speed. The irregular deviation of the downhole speed shows the movements of the drill string. When the surface and downhole speeds are almost the same, the drill string is basically rotating at the center of the wellbore but when the drill string revolves around the wellbore, its downhole speed changes in reaction to this anomaly. 


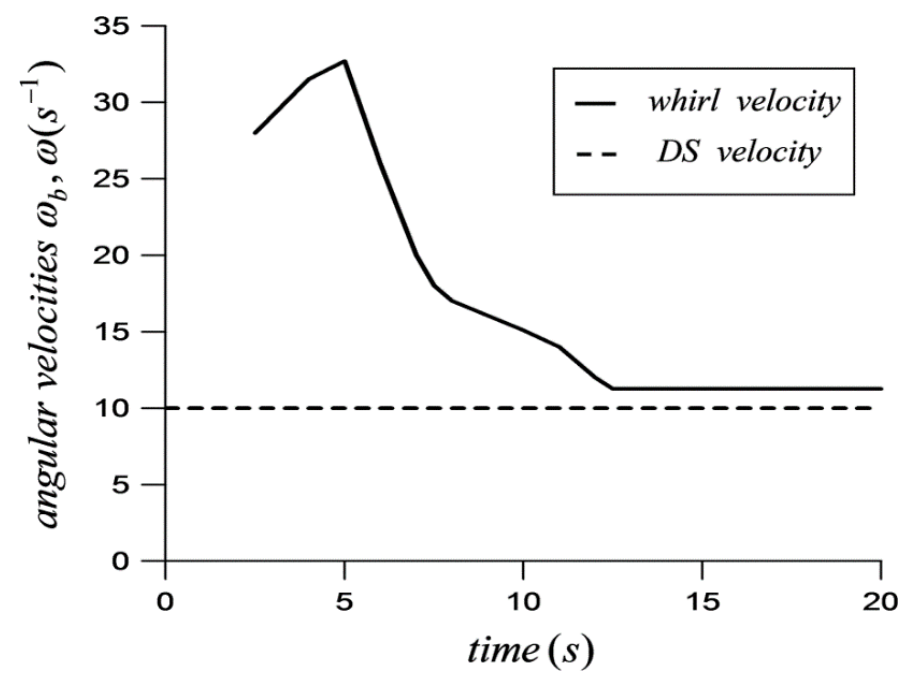

Figure 4 Effect of Whirling on Downhole RPM [7]

Figure 5 shows two basic kinds of whirling. First is forward whirling where the revolving of the drill string around the wellbore is in the same direction as the rotation of the drill string around its centerline. The other type of whirling is backward whirling where the drill string revolves around the wellbore is a direction opposite the direction the drill string rotates around its centerline. These two types of whirling are studied in this research.
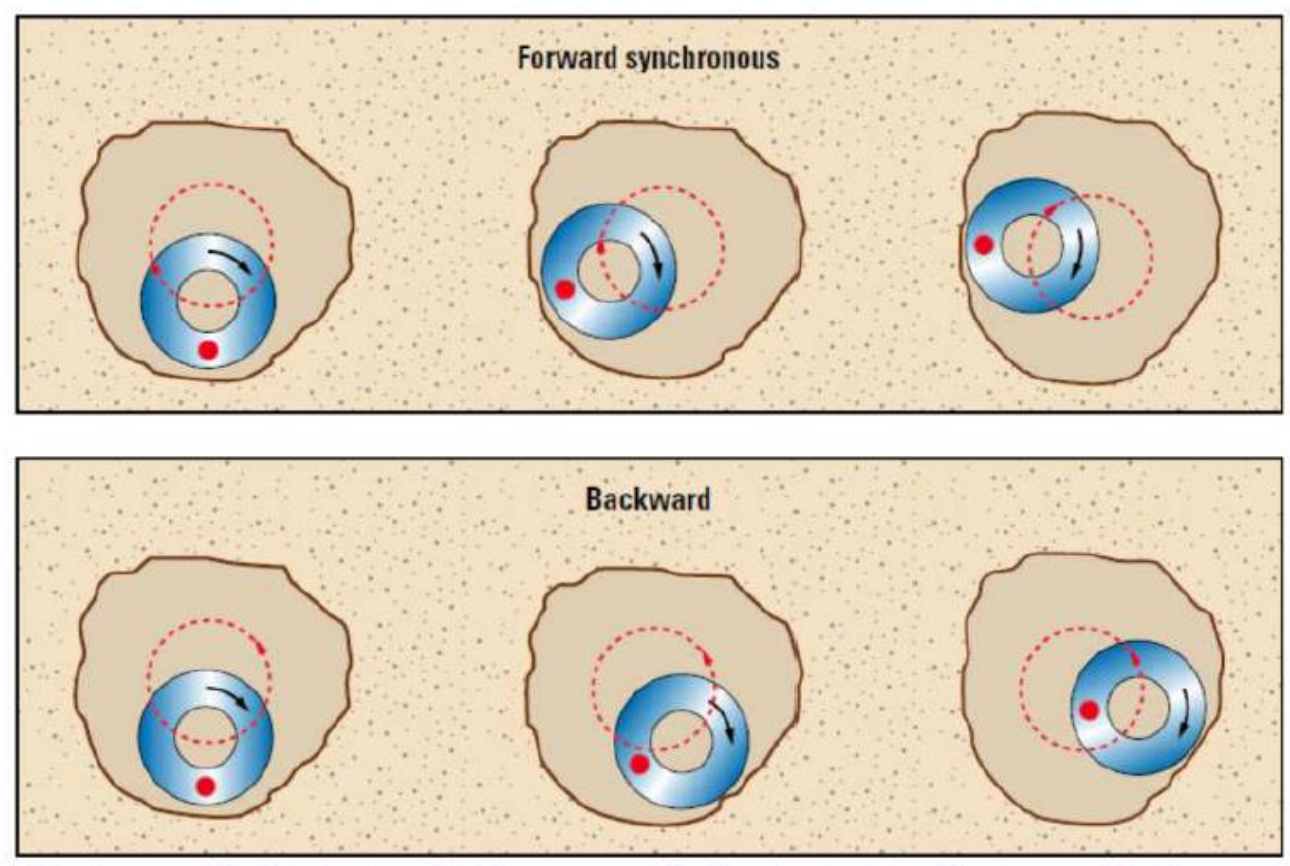

Figure 5 Forward and Backward Whirling [3] 
There is a third scenario where the drill string revolves in a chaotic manner. This is called chaotic whirling. In this case, the revolving and the rotation happen at irregular patterns with no distinct direction. Whirling could cause fatigue on the drill string eventually creating bending stress. Axial Vibration

Occasionally, the drill string may bounce as the cutters hit the formation. This phenomenon is known as bit bounce. Continuous bit bounce makes the drill string to vibrate longitudinally in the axial direction. When the WOB is frequently changed by the driller when the hook load at the surface is adjusted, this mode of vibration is stimulated. It typically occurs in roller cone bits and it is also caused by sudden variations in mud pressure [4]. This type of vibration occurs early on in the drilling process in shallow wells. It occurs when there is a quick interchange of hard and soft formations [5].

\subsubsection{Coupling of Vibration Modes}

A lot of complexities exist in axial and torsional vibrations; they could occur solely but they coexist. During stick-slip torsional motion, the high RPM level may cause severe axial vibrations, which in turn could lead to bit-bounce, bit wear, and low ROP [79]. The sticking phase of stickslip would spur an instantaneous maximum downhole RPM which may be up to twice the surface RPM. This would lead to a high centrifugal force which can stir up backward whirling even though the surface RPM is low [8]. A driller's dilemma emerges when increasing weight-on-bit (WOB) induces stick-slip whereas increasing revolutions/minute (RPM) induces whirl. Figure 6 shows the directions these three types of vibrations go when they occur. 


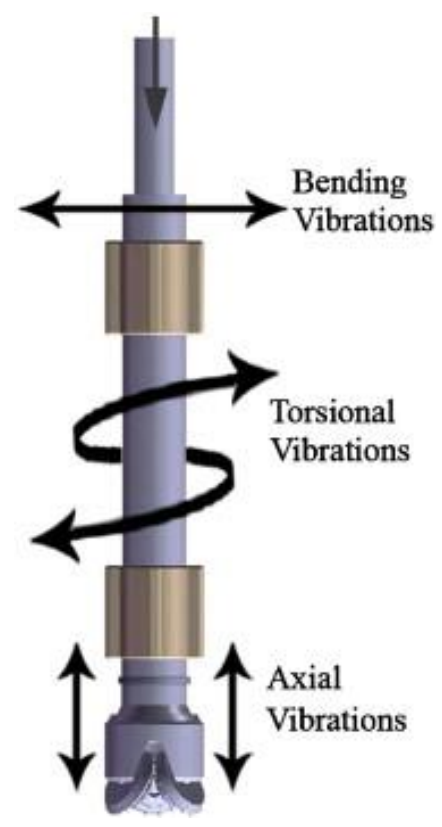

Figure 6 Simultaneous Vibrations [9]

\subsection{Research Objectives and Contributions}

The objective of this research is to describe a process that informs the driller of optimized drilling parameters before, during and after vibration occurs downhole using the optimum zone chart. The optimum zone chart is a plot of WOB and RPM with data points forming an envelope which excludes vibration prone zones. Five zones can be identified from the optimum zone chart; forward whirling zone, backward whirling zone, stick-slip zone, low ROP zone and the optimum zone. The process of identifying the optimum zone on a time periodic basis is discussed. This process is the use of different machine learning techniques to analyze data from a well. The findings at each stage of the process is summarized. The research also reviews the feasibility of creating a startup company if the process identified by this research is considered a software as a service business. An evaluation of the market is done with detailed competitor analysis. A detailed business model that would make this startup viable is described.

The contributions of this research are twofold. On the technical end, the optimum zone chart is created based on surface data alone. This means vibrations can be detected and mitigated without using downhole sensors and more importantly in real time. That is a major contribution when one 
considers the cost of getting vibration information from downhole sensors owned by the service companies. On the business end, the market research done would help understand the best way the product from this research can be deployed into the industry. Potential customers were recommended and how best to approach these customers were advised. An innovative way of running the business was also discussed as a blue ocean strategy.

\subsection{Structure of Thesis Report}

This report starts with basic understanding of the drilling process, an overview of the process of developing the optimum zone is discussed and the discoveries from interviews with potential customers are highlighted. The results of testing the algorithms on a well is summarized. Recommendations on the pathway to building the startup are made.

Chapter two gives the literature review of past methods used to reduce vibrations of the drill string. Most authors investigate the different vibration modes on a standalone basis. This chapter will look at results of research for reducing stick slip, whirling and summarize value derived from tools and software programs currently in use to measure and mitigate vibrations. Since machine learning is the method in use for this research, the literature review is extended to knowing what achievements have been made in the oil and gas industry while using machine learning.

Chapter three reviews each machine learning step taken to derive the optimum zone. The theory involved is explained and examples are shown for better understanding. The data acquisition process is also explained. Two options for receiving real time data are discussed in detail.

Chapter four looks at the process of optimizing drilling parameters using optimum zone chart. The individual machine learning processes are applied to generate the optimum zone chart. The conventional way of curbing vibrations is first reviewed then the machine learning method is then formulated with basic tweaks of the conventional method. The concept of a safe zone is introduced. Chapter five gives an analysis of the result while using historical data (post analysis) and proposes a real-time scenario. The results are presented for every stage of the process leading to the optimum 
zone. The results based on the safe zone concept is compared to results with just the optimum zone.

Chapter six presents the feasibility of running the product of this research as a startup company. The result from customer discovery and customer validation are presented. A value proposition canvas is developed and used as the basis for a business model canvas. A detailed competitive analysis is discussed.

Chapter seven highlights observations from the process of using machine learning to generate the optimum zone of the well. These conclusions also come from the results of testing with historical field data. The important findings from customer discovery and validation interviews are summarized. A summary of how the startup from the research will run is given. Recommendations are made for further work on the technical process and business options. The next steps on the technical side and on the business side are also highlighted. 


\section{Chapter Two: LITERATURE REVIEW}

Since vibrations have been known to negatively affect the drilling process, a lot of work has gone into research to solve this problem. This chapter summarizes some of these researches from professionals in the drilling industry and professors and students in drilling related university research. Downhole tools and software used to reduce vibrations is also reviewed. A look into how machine learning has been previously used in the drilling industry is also discussed.

\subsection{Stick Slip Mitigation Techniques}

In a bid to find out the effects of stick slip on rate of penetration, ROP, Challamel et al in 2000, collated data from instrumented PDC bit during a drill-off test. They introduced a measuring tool which they placed above the bit and evaluated the effects of stick slip on torque on bit, weight on bit and accelerations [10]. Kyllingstad et al in 2009 introduced a new NOV technique for mitigating stick slip by simply tuning a proportional integrator (PI) speed controller to dampen stick-slip torsional vibrations. The new technique involves the use of softspeed which can tell the amount of bit speed variation. During stick-slip, the driller could turn on the softspeed, which means the drive will show dynamic variations as a result of torque variations. The stick slip oscillations will consequently fade away [81]. In 2011, NOV used their vibration test rig to simulate stick slip vibrations so they can test different mitigation techniques. They eventually discovered that by inducing axial vibration down the drill string, other tools to mitigate stick slip worked better and stick slip was significantly reduced [11]. Also in 2011, Zakuan et al described how roller reamer can be used to mitigate stick slip. They explained that the roller cutters in the roller reamer would reduce torque while rotating around the wellbore in a stick slip event. This would mean low friction between the drilling string/the bottom hole assembly (BHA) and the wellbore wall. They estimated that their technique would save $40 \%$ of the drilling time [12]. A summary of the usage of Shell Soft Torque Rotary System (STRS) was highlighted by Runia et al in 2013. The STRS achieves stick-slip mitigation by modifying the rotary drive control system 
used in oil and gas drilling. The concept is to modulate surface rotary speed in order to eliminate torsional vibrations (stick-slip) [80]. The design of the bit in use can also cause stick slip and in 2014, Schwefe et al discovered that when depth-of-cut technique is used to design PDC bit, bit induced stick slip can be reduced significantly. This method is more effective when compared with other PDC design techniques [13]. What if stick slip could be monitored and when required, it is reduced by mitigation tools? These were the questions answered by Efteland et al in 2015 when they proposed the use of a remote monitoring system that would guide mitigation tools during stick slip problems. Their monitoring system could identify stick slip before the crew at the surface could see it. This system allowed for increase in WOB without further increase in stick slip [14]. Also in 2015 Dwars outlined the strength of Shell's softtorque technology but also mentioned that the constant re-tuning of the top drive system would be error prone and very laborious. Hence the need for improving the technology. Dwars introduced this improvement as the "ztorque technology." Z-torque seeks to decrease torsional energy reflection at the surface back to the drill string by utilizing electronics engineering design concepts and applying them to the top drive and drill string [82].

There have been attempts to automate the process of mitigating stick slip. In 2016, Vogel et al developed an automated system that could reduce stick slip and hence reduce drilling time. This was done using surface data to detect stick slip severity and change top drive RPM as a response [15]. In 2017, Shen et al desired to understand how severe stick slip occurs; they conducted a series of tests using a high tech downhole measurement tool on a drilling system modeled on a 3D transient simulation framework with accurate wellbore geometry. They concluded that a good understanding of the process involved during stick slip will give greater opportunities to control severe stick slip; they also realized that breaking the mechanism can decrease the severity of stick slip [16]. Bailey et al in 2018, showed how drill string and drilling parameters can be redesigned based on stick slip vibration distributions to reduce stick slip on new wells. They deeply studied relations between vibration distribution function and other variables like RPM, downhole torque. 
The results helped the drillers understand the degree stick slip vibration that could occur in subsequent wells [17].

\subsection{Attempts to Curb Whirling}

In 1990, Vandiver et al identified two causes of BHA vibration as whirling in drill string and fluctuations of WOB coupled with bending of an initially curved BHA. They propose equations

for forward and backward whirl rate. They suggested that downhole measurement of bending moments can be used to detect whirling vibration [18]. Zhang et al in 1992 were able to calculate the natural angular frequencies of lateral vibration (NAFLV) of rotating drill string joints. They verified their results using simulation tests. Their findings were based on the principle of selfexcited whirling [19]. Rey-Fabret et al in 1997 noted that there are no means of detecting whirling from the surface even though whirling causes breakdown of the drill string and destruction of the bit. They explained that two changes indicate whirling occurrence: a shift in the average rotary torque and variation in weight on hook measurements. They introduced a software to reflect these changes based on surface measurements [20]. Hutchinson et al in 2013 described a way to mitigate whirling by inserting a shock sub into the BHA or inserting a torque-limiter to continuously monitoring vibrations and then given signals the driller at the surface for parameter changes whenever downhole shock levels are too high. They show an innovative self-adaptive vibration damper which detect dysfunction due to whirling and then adjusts the device's damping features until the bad motions are mitigated [21]. Marquez et al in 2017 showed that whirling arising from rock-bit interactions can be minimized by use of finite element analysis (FEA) tool. Their study investigated coupling of axial, lateral (whirling) and torsional vibration using the FEA to reduce whirling in the drill string by optimizing the position of stabilizers and the number of stabilizers needed [22]. Also, in 2017, Zhao et al developed a mechanism to detect precursors of drilling events such as severe whirling vibration. They used machine learning algorithms to figure out what changes in drilling parameters continuously occur when whirling vibration occurs or any other 
drilling event occurs. They then represent the drilling parameters in time series using symbolic aggregate approximation (SAX) [23].

\subsection{Overview of Technologies for Mitigating Drill String Vibrations}

Based on cable tools and sucker rods, Slonneger in 1937 explained an easier way of estimating vibration frequencies and plotted chart for these frequencies [24]. In 1960, a new kind of measurement equipment was described by Bailey et al. The equipment could measure axial force, torque, and axial and rotational motions at the top of a drill string [25]. During the mid-60s, Deily et al collected data from fifteen wells considering diverse drilling conditions. They used a downhole recording instrument to track motions and forces of the drill string. The eight signals recorded by the pulse-width modulation on the magnetic tape were: axial, torsional, and bending loads; axial, angular, and lateral accelerations; and internal (pipe) and external (annular) pressure [26]. An analysis of their work done also in 1968 by a Staff Engineer at Hughes Tool Company who noticed that the tests made by Deily et al confirm and give quantitative values to many phenomena which have been observed qualitatively or which have been indicated by existing evidence. Cunningham observed that the phenomena that were distinctly shown to exist in some cases were irregular bit rotation, torque fluctuations, bit bouncing, bit weight fluctuations, and large losses of rotary horsepower along the drill stem [27].

The interface between the formation and the drill string were highlighted by Skaugen in 1987 as a primary source of vibrations of the drill string. He observed measuring forces within the BHA show how bit vibrations have enormous quasi-random components, both for axial and rotational movements. The vibrations were caused by patchiness of the formation strength and breakage of the formation, and amplification of these effects by vibration mode coupling [28]. In 1992 Langeveld et al noticed that vibrations downhole caused reduced efficiency of PDC bits in tough formations. They underlined several ways to resolve the issue: reduce RPM, increase WOB, use anti-whirl bits and adopt a soft-torque rotary system to extend the usability of the PDC bits across various formations. These conclusions were based on modelling the bit/drill string assemblies and 
supported by advances in PDC bit technology related to torsional vibration control systems [29]. A computer program based on Fourier Spectral Analysis was utilized by Rajnauth in 2003 to provide real time display of the scale of torsional vibration on the data from the drilling sensor for two wells [30]. Detecting the potential for vibration during drilling was done in 2009 by Craig et al using computational and analytical models. They achieved this by identifying the various RPMs and WOB where the BHA/drill string will resonate in the axial, lateral, and torsional directions for different depths along the well path [31]. In 2014, Mario et al noted that drilling efficiency could be improved by mitigating vibrations using roller reamer in the BHA. They observed positive results and reduction in well cost and non-productive time, NPT [32]. Greenwood in 2016 described several factors that cause drill string vibration: the energy inputs of WOB and torque, type of lithology, type of bit, rock bit interactions, wellbore size, structure of the formation, dimensions of the BHA, BHA design and stabilization, hole trajectory and many more. The methodology he used was able to incorporate time based analyses and depth based logs in order to identify and isolate which factors are the root cause of a specific vibration mechanism He then plotted a to-scale BHA to identify correlations of the position of the bit, stabilizer, and other BHA components to casing positions, formation types and boundaries hole-size changes [33]. Also in 2016, Jeremy showed that incorrect measurements of drilling parameters obscure the vision of the driller to the existence of damaging vibration and can potentially lead low performance and damages of downhole tools. He introduced a smart system which integrates downhole and surface measurements to help the driller to correctly identify the kind of vibration occurring and know what to do in response [34].

\subsection{Software for Identifying Vibration Problems}

In 2003, Rajnauth et al analyzed a software package used on wells drilled off the East Coast of Trinidad. Their software worked based on Fourier Spectral Analysis of drilling data to show the severity of stick slip vibrations. They noticed that the vibrations had great impact on the bit and other downhole tools [35]. Craig et al in 2009 described a software developed by NOV which 
allows users to construct a finite element model of the BHA and drill string system using input from geometric and material definitions of the individual components of the drilling profile. This software also shows the user different RPMs that will vibrate in axial, lateral and torsional directions based on various WOB chosen by the user at different depths along the well path [36]. In 2012, Wu et al working with Schlumberger combined expertise in advanced drilling dynamics model to successfully identify the causes of vibration in many drilling programs worldwide. This would help drilling engineers focus on the exact problem going on downhole rather than on some trial and error approach [37]. Also in 2012, Amorim et al developed a software using Finite Element Analysis (FEA) made for understanding vibration downhole and accurate prediction of operating parameters for efficient drilling. The software works based on the dimensions of the BHA and the design of the wellbore [38]. Hanley et al in 2012 described the development of software which provides the user with an in-built suite of data analysis and drilling optimization systems. The software system was modeled to operate swiftly on key business and analytical processes to ensure reduction of vibration issues [39]. In 2014, Song et al described an integrated drilling engineering software developed for drilling planning and real-time optimization to avoid vibration and to accomplish the needs of drilling design, analysis and construction optimization. The software contains a drilling risk diagnosis and processing subsystem which gets reasonable risk threshold through data patterning and training using neural network algorithm, then inputs the drilling risk factors while drilling, finally analyzes and forecasting the drilling risks for parts of the trajectory yet to be drilled [40].

\subsection{Machine Learning Usage in Research}

One unique method that can be applied to optimize the drilling process is "machine learning." Machine learning is not new; it has been around at least since the 1970s, when the first related algorithms appeared. The general idea behind most machine learning is that a computer learns to perform a task by studying a training set of examples. The computer (or system of distributed or embedded computers and controllers) then performs the same task with data it hasn't encountered 
before [41]. This method is primary focus of this paper. Machine learning has been applied to other aspects in the oil industry. Zhang et al in 1991, applied machine learning to rock mechanics and observed that all of the factors governing the rock mass behaviors could be considered as input variables to predict the varying rock behaviors without putting a cap on the amount of these variables inputted if the training examples are available enough [42]. Vladimir et al in 2002 used machine learning in their aim to adapt EOR technologies to rejuvenate many mature fields in Venezuela. They used machine learning algorithms to draw rules for screening [43]. In 2016, Sarkar et al applied machine learning for the prediction of tidal currents. They concluded that the algorithm they used would specifically help harness energy from location of fast tidal currents [44]. In 2017, Bangert proposed the use of machine learning to conduct smart condition monitoring. He realized that his proposed method was more successful than standard condition monitoring thus preventing false alarms and always alarming unhealthy states of plants or equipment [45]. This research aims to use machine learning principles to help the driller make quality decisions in terms of drilling parameter adjustments during vibration problems thereby optimizing the drilling process.

\subsection{Chapter Two Conclusion}

In this chapter some technologies for reducing vibrations downhole have been reviewed. In one instance, axial vibrations were induced to mitigate stick slip vibrations. Two main causes of whirling were identified. Whirling would occur when there is frequent change in the average rotary torque and when there is variation in weight from hook measurements. Some other causes of vibration in the drill string are unevenness of formation strength and breakage of the formation. 


\section{Chapter Three: USING MACHINE LEARNING TO IDENTIFY VIBRATION}

In this chapter, the basics of machine learning is explained and the detailed explanation of what machine learning tools are used to curb vibration is explained.

\subsection{Basics of Machine Learning}

Machine learning gives computers the ability to optimize a performance criterion based on sample data or past knowledge [46]. The goal of machine learning is to identify and reveal potential veiled patterns linked with the data being analyzed. The world today is circled with applications of machine learning. A perfect example is the use of Google search which learns to display the best results. Another example is the anti-spam software which filters email messages [47].

As shown in Figure 7, there are two major types of machine learning. First is supervised (predictive) learning where for a given input variables $(\mathrm{x})$ and output variables $(\mathrm{Y})$, the predictive algorithm maps out patterns in the input data and forms a way to predict the output for subsequent input data $Y=f(x)$. Unsupervised (descriptive) learning is the second major type of machine learning. Unsupervised learning is where for a given input data (x), there are no corresponding output but the algorithm is written to find certain patterns in the input and then generate outputs.

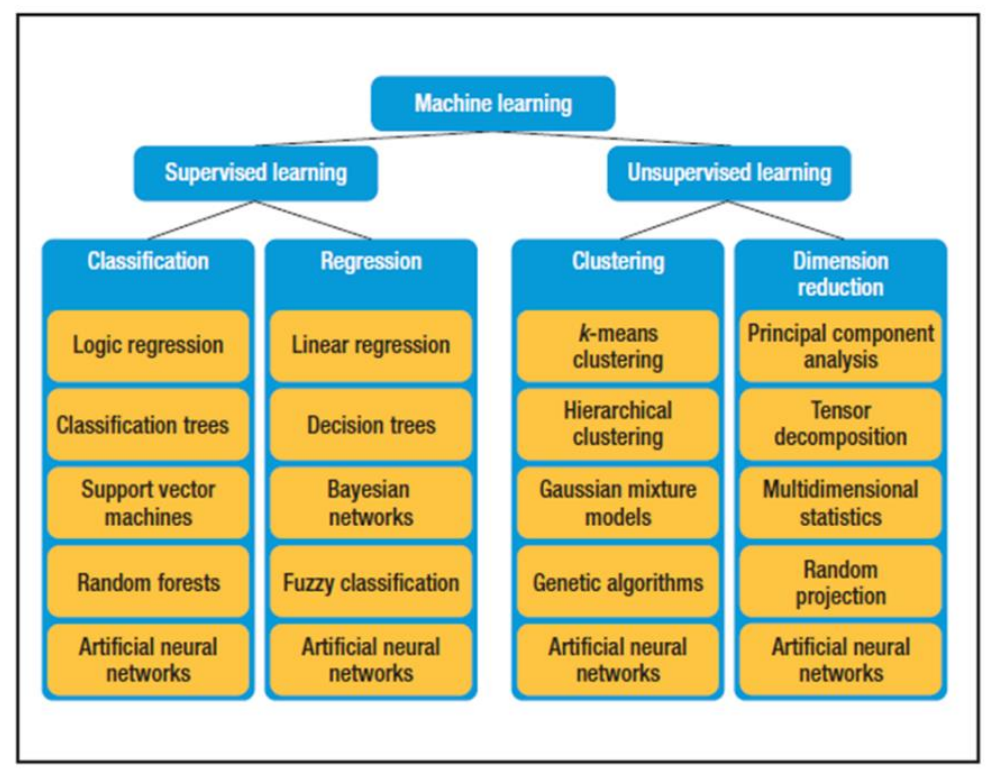

Figure 7 Machine Learning Methods [41] 


\subsection{The Process}

The two most important parameters that indicate the occurrence of vibration problems downhole are weight on bit (WOB) and rotational speed (RPM) [85]. Previous investigators have developed a chart that shows the safe drilling window. This chart of WOB versus RPM can be used to show the driller what WOB and RPM values are safe during vibration problems. All the driller needs to do is pick values within the optimum zone.

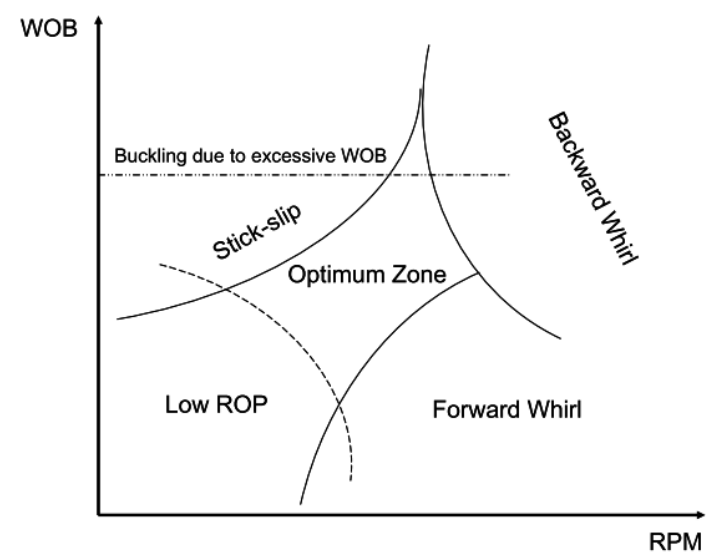

Figure 8 Optimum Zone Chart [48]

The goal is to reduce the vibrations and optimize rate of penetration (ROP). The hard question to answer is if the stick slip, and whirling zones are predicted accurately. Thus, the need for machine learning. In order to identify the optimum zone effectively, a process, as shown in Figure 9, is adopted to ensure all drilling parameters have an impact on the optimum zone. The process uses available real-time data from the drilling rig, perform a variable transformation and reduction, and then utilize machine learning algorithms to identify the optimum zones. The zones are then transformed back into the original variables and displayed to the driller. Principal Component Analysis (PCA) is used to achieve the variable transformation and reduction. Decision tree classification is used to classify the data points into the different zones: stick slip, forward whirling, backward whirling, low ROP and optimum zone. Kmeans clustering is then used to identify the exact WOB and RPM the driller is to work with per update of the optimum zone. 


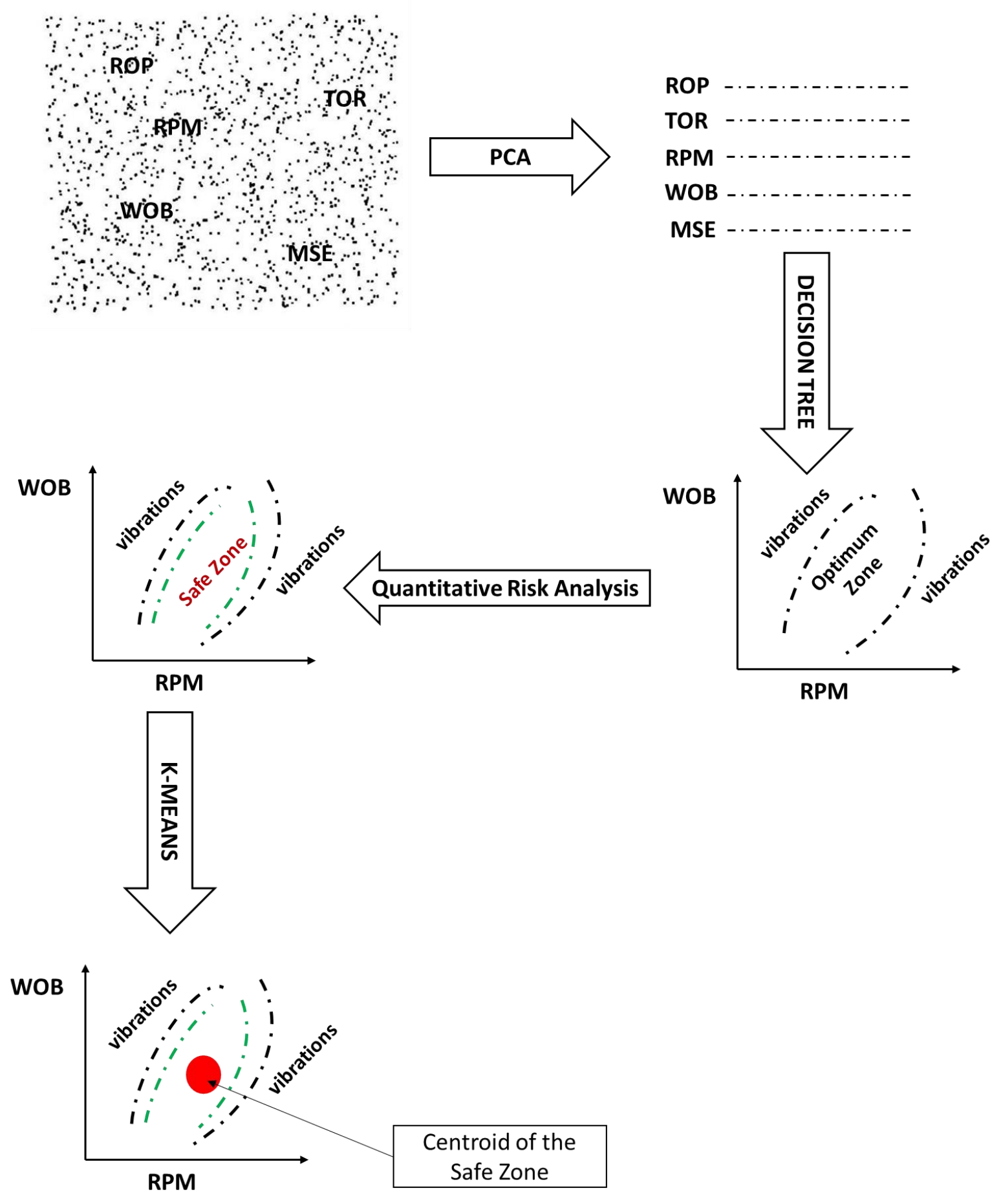

Figure 9 Using Machine Learning to Identify Optimum Zone and Safe Zone 


\subsection{Useful Drilling Parameters}

Apart from WOB and RPM values needed, other drilling parameters affect the entire drilling process and consequently the impact vibrations of the drill strings. The table below shows some of those parameters. The list could change for every new well depending on what parameters are in need for optimization.

\section{Table 1 Drilling Parameters and their Significance}

\begin{tabular}{|l|l|}
\hline Drilling Parameter & Significance \\
\hline $\begin{array}{l}\text { Unconfined Compressive } \\
\text { Strength (UCS) }\end{array}$ & $\begin{array}{l}\text { It is the maximum axial compressive strength a rock can } \\
\text { withstand under unconfined conditions [49]. }\end{array}$ \\
\hline Revolutions per Minute (RPM) & $\begin{array}{l}\text { It is becoming a rule of thumb that higher bit RPM leads } \\
\text { to more opportunities to cut deeper into the formation per } \\
\text { time [50]. }\end{array}$ \\
\hline Rate of Penetration (ROP) & $\begin{array}{l}\text { In every foot of hole drilled, the vision of every operator is } \\
\text { to have a management process for the drill rate maximize } \\
\text { ROP [51]. }\end{array}$ \\
\hline Weight on Bit (WOB) & $\begin{array}{l}\text { Better decision that results in lower cost per foot are being } \\
\text { made based on information derived from downhole WOB } \\
\text { [52]. }\end{array}$ \\
\hline D-Exponent (d) & $\begin{array}{l}\text { The D-exponent is used to account for frequent changes in } \\
\text { values of hole size, RPM and ROP, the D-Exponent is } \\
\text { calculated [53]. }\end{array}$ \\
\hline Torque (TOR) & $\begin{array}{l}\text { Every well has a limit for drilling torque but in the past most } \\
\text { wells have suffered excessive torque leading to unfeasible } \\
\text { projects due to unusable wells [54]. }\end{array}$ \\
\hline
\end{tabular}

Using principal component analysis (PCA), a dimension reduction process, the dependence of the input WOB and RPM on other input drilling parameters can be reflected on the optimum zone chart. In order to generate the optimum zone in real time, the data required in this research is restricted to surface data. There are two ways to connect to surface data in real time: one is to connect to an Electronic Drilling Recorder (EDR) made by companies like Pason; another way is to connect to the rig's operating system made by companies like NOV (NOVOS).

\subsubsection{Electronic Drilling Recorder}

The EDR is a drilling data recorder which also a network of sensors and software programs which informs rig workers of data changes in real time. 


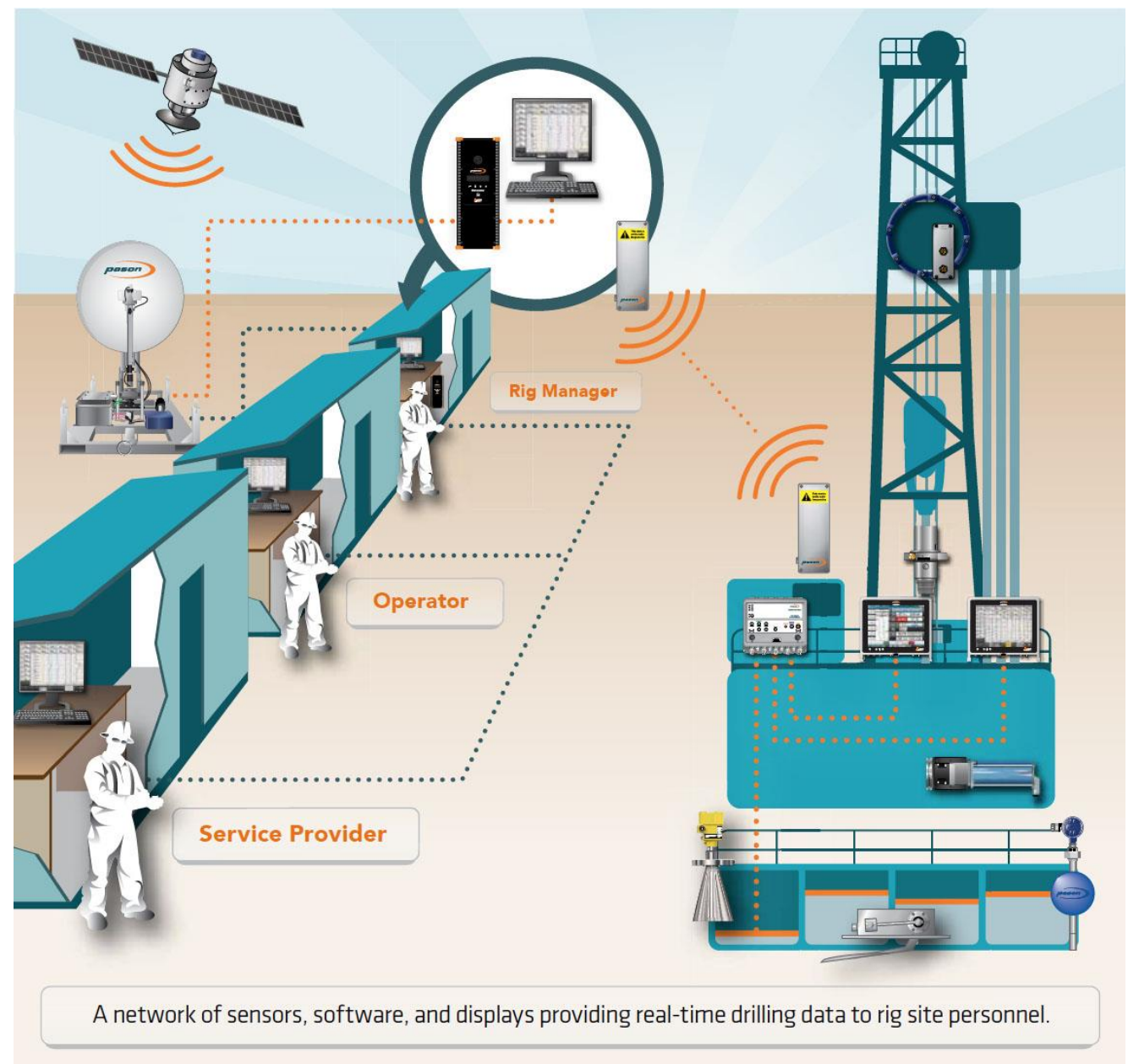

Figure 10 The Flow of Information using Pason EDR [55]

The EDR integrates data collation among engineers, geologist and technicians on the rig. With the EDR, they can all work on the same data platform [55]. Through the use of satellite, the data on this platform can be shared with offsite office users and remote centers. Drilling reports can also be shared via this route. The optimum zone chart can relate with the EDR using the Wellsite Information Transfer Specification (WITS) protocol.

\subsubsection{Rig Operating System}

The rig operating system is a process automation channel where two activities are linked in order to relieve the driller of the burden of manually connecting these activities. The operating system is built with control, monitoring drilling optimization and scheduling. Most rig operating systems, like NOVOS, can be installed in just half a day on a rig [56]. To connect the optimum zone chart 
to the rig's operating system, an app has to be build which will be compatible the particular brand of rig operating system in use at the time.

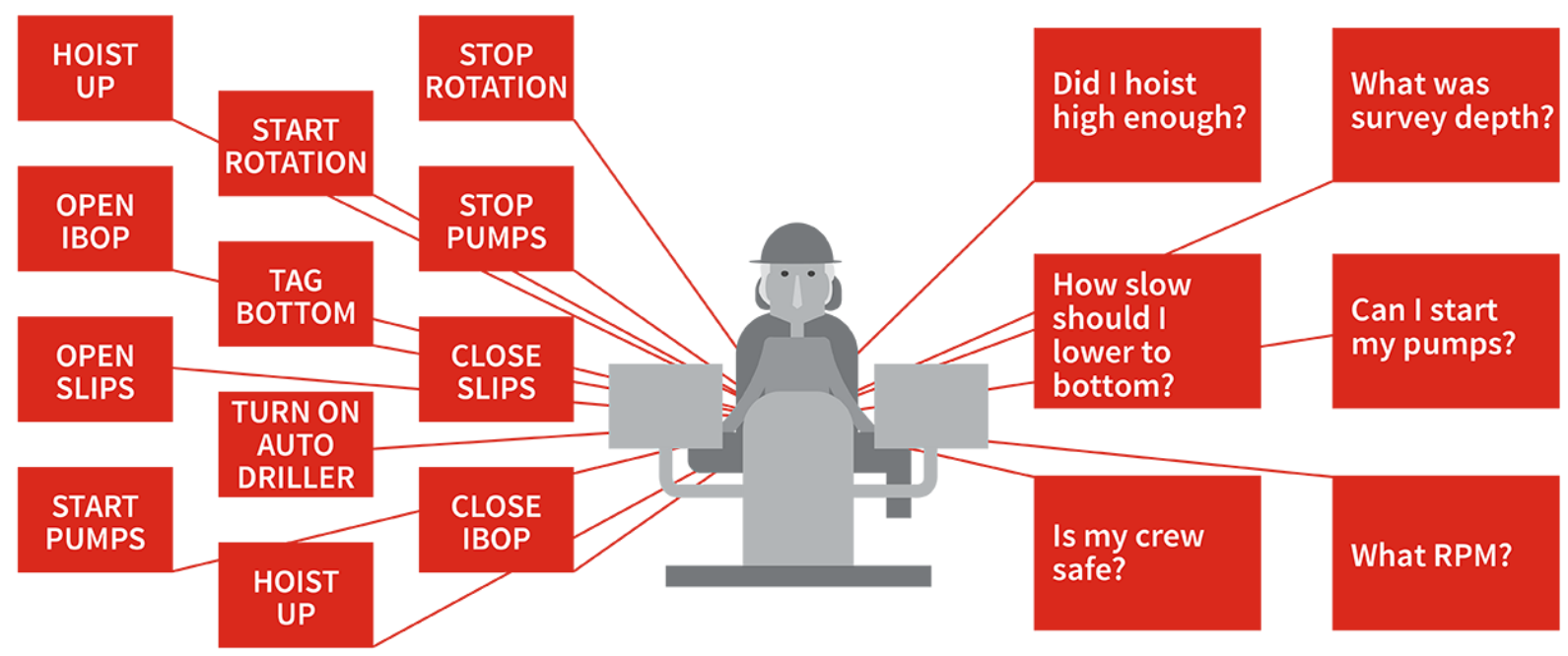

Figure 11 Functionalities of NOVOS [57]

\subsection{Principal Component Analysis (PCA)}

PCA can be used for searching out veiled patterns in high dimension data (the number of features exceed the number of observation); its most common application is in image compression. In this research, PCA is used for reducing the dimension of the input data without losing important information in the original data [58]. Three steps govern the PCA process.

- The first step is to determine the covariance of the matrix. Covariance is the measure how two different variables relate with each other during changes in values. The formula for covariance is an adjustment of the variance formula which only analysis the dataset in one variable.

$$
\text { Variance }=\sigma^{2}=\frac{\sum(x-\mu)^{2}}{N}
$$

For variable $x, \mu$ is the mean and $\mathrm{N}$ is quantity of data points in variable $x$. This formula is then modified the give the formula for covariance between two variables. Consider two variables $x$ and $y$

$$
\text { Covariance }=\operatorname{cov}(x, y)=\frac{\sum_{i=1}^{n}\left(x_{i}-\bar{x}\right)\left(y_{i}-\bar{y}\right)}{n-1}
$$


If multiple variables are involved, the covariance matrix will be symmetrical; meaning the transpose of the matrix will be the same as the original matrix. Assuming there are four variables, w, $\mathrm{x}, \mathrm{y}$ and $\mathrm{z}$. The covariance matrix will be as follows:

$$
C=\left(\begin{array}{cccc}
\operatorname{cov}(w, w) & \operatorname{cov}(w, x) & \operatorname{cov}(w, y) & \operatorname{cov}(w, z) \\
\operatorname{cov}(x, w) & \operatorname{cov}(x, x) & \operatorname{cov}(x, y) & \operatorname{cov}(x, z) \\
\operatorname{cov}(y, w) & \operatorname{cov}(y, x) & \operatorname{cov}(y, y) & \operatorname{cov}(y, z) \\
\operatorname{cov}(z, w) & \operatorname{cov}(z, x) & \operatorname{cov}(z, y) & \operatorname{cov}(z, z)
\end{array}\right)
$$

Note that the diagonal are the variances of each variable.

- Next would be to estimate the eigenvalues and eigenvectors of the covariance matrix.

Let $\mathrm{A}$ be an $n X n$ matrix. The number $\lambda$ is an eigenvalue of $\mathrm{A}$ if there exist a non-zero vector v, such that $A v=\lambda v$

The eigen values of A are the roots of the characteristic polynomial $p(\lambda)=\operatorname{det}(A-\lambda I)$; where $I$ is the identity matrix. $I=\left(\begin{array}{lll}1 & 0 & 0 \\ 0 & 1 & 0 \\ 0 & 0 & 1\end{array}\right)$ or $I=\left(\begin{array}{ll}1 & 0 \\ 0 & 1\end{array}\right)$ For each eigenvalue $\lambda$, the corresponding eigenvectors are $v=\left[\begin{array}{c}v_{1} \\ v_{2} \\ \vdots \\ \cdot \\ v_{n}\end{array}\right]$ by solving the linear system $(A-\lambda I) v=0$

- The principal components are the eigenvectors. The principal components are ranked according to their corresponding eigenvalues. If the characteristic polynomial of A has 4 as its highest power then there would be 4 eigenvalues. The highest eigenvalue would produce the first principal component; the second highest eigenvalue would produce the second principal component (eigenvector).

In Figure 12, the data is first plotted on $\mathrm{X}$ and $\mathrm{Y}$ coordinates. The principal direction is where the highest variance lies. In this case, the $U$ direction is the principal direction with the highest importance. The $\mathrm{V}$ direction must be orthogonal to the $\mathrm{U}$ direction. It is expected that when $\mathrm{X}$ and $\mathrm{Y}$ coordinates are transformed into $\mathrm{U}$ and $\mathrm{V}$ coordinates, the covariance between $\mathrm{X}$ and $\mathrm{Y}$ variables 
becomes zero. $\mathrm{U}$ and $\mathrm{V}$ variables are called principal components [59]. In reality, they are the eigenvectors of the covariance matrix of the original dataset. The level of importance is based on the eigenvalues; the eigenvector with the highest eigenvalue is the most significant and is termed the first principal component. The eigenvector orthogonal to the first principal component with the next highest eigenvalue is the second principal component and so on [59]. The reduction aspect is done after the original dataset has been transformed to principal components. Before inverse PCA is done to get the original variables, some dimensions are zeroed out which have low eigenvalues. The resulting original dataset is leaner and very distinct on what values are to be used as shown in Figure 13.

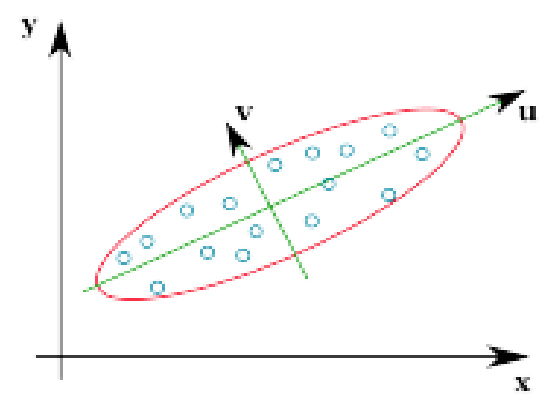

Figure 12 PCA for Data Representation [59]

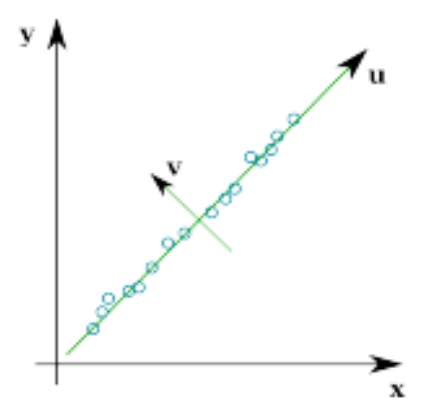

Figure 13 PCA for Dimension Reduction [59]

\subsection{K-means Clustering}

K-means clustering is a type of unsupervised learning where algorithm searches the data for groupings and the stability of these groupings. After several iterations, the data is divided into K 
number of stable clusters [60]. The input parameters are the number of clusters, $\mathrm{K}$, and the dataset. The algorithm keeps iterating the positions of $\mathrm{K}$ clusters and keeps readjusting the data points in one cluster until a stable position for all the centroids is achieved. There are two governing equations for this process.

1. The squared Euclidean distance equation is used to assign each data point to one cluster. This is done by assigning each data point to the cluster where it is closest to its centroid. $\mathrm{c}_{\mathrm{i}}$ is one centroid in a set of centroids $\mathrm{C}$. $\mathrm{x}$ represents the data points and it is being assigned to each cluster based on

$$
\arg \min _{c_{i} \in C} \operatorname{dist}\left(c_{i}, x\right)^{2}
$$

Where $\operatorname{dist}(\cdot)$ is the Euclidean distance and $S_{i}$ is the data point for the $i^{\text {th }}$ cluster

2. The centroid calculation equation is used to calculate the centroid for each cluster which is the mean of all data points in that cluster [60].

$$
c_{i}=\frac{1}{\left|S_{i}\right|} \sum_{x_{i} \in S_{i}} x_{i}
$$

There are 3 criteria to stop the iteration

- No movement of data points between clusters

- Minimized sum of distances

- A set limit to the number of iterations is reached

Mean distance of the data points to the centroid is plotted against $\mathrm{K}$ and the "elbow point," where the rate of decrease sharply shifts or the average mean distance becomes constant, is a way to determine the value of $\mathrm{K}$ as shown in Figure 14 [86]. 


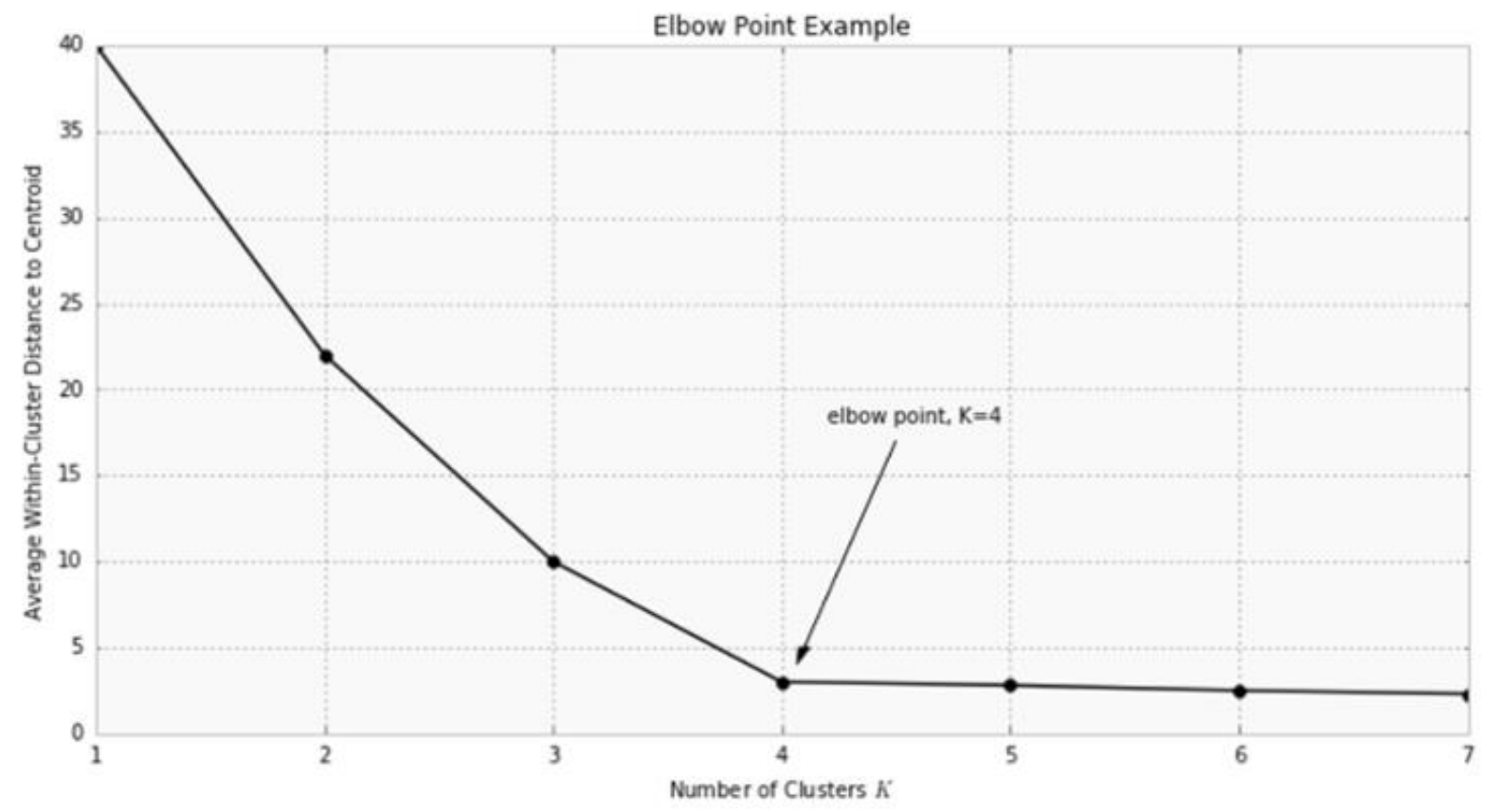

Figure 14 Elbow Point Method for Determining the Value of K [86]

\subsection{Decision Tree Classification}

Decision Trees are a type of supervised learning where the algorithm ensures thorough searches into the input data to find patterns that place each data point into target variable. The learning is on the rules within the data that determine how each data point is placed on a target variable [61]. To build the decision tree, several questions and possible answers are placed in a hierarchy. Data points that meet the specific requirement at each point will classified into that variable [61]. Figure 14 is an example of classification done for mammals. Just like all decision trees, this tree has three types of nodes: 
Table 2 Types of Nodes in a Decision Tree and their Characteristics

\begin{tabular}{|l|l|l|l|l|}
\hline Node Type & Incoming Edge & Outgoing Edge & Description & Shape \\
\hline Root or Decision Node & None & Zero or More & $\begin{array}{l}\text { Decision has been } \\
\text { made }\end{array}$ & Square \\
\hline Internal or Chance Node & One & Two or more & $\begin{array}{l}\text { There are at least two } \\
\text { possible outcomes }\end{array}$ & Circle \\
\hline Leaf or Terminal Node & One & Zero & $\begin{array}{l}\text { It does not have child } \\
\text { nodes }\end{array}$ & Triangle \\
\hline
\end{tabular}

The end variables are assigned to the terminal node. The questions and answers are assigned to the root and internal nodes. This can be seen in the example below.

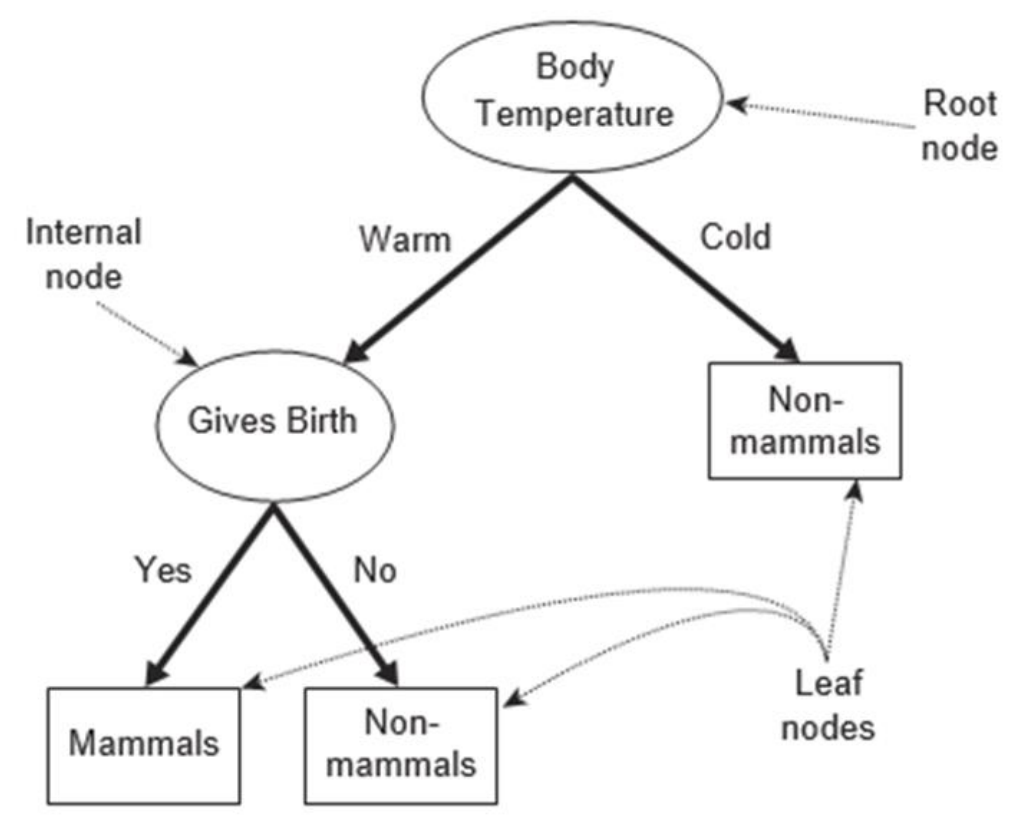

Figure 15 Decision Tree for Mammal Classification [62]

The decision tree is constructed based on 3 generic steps:

- Step 1: Place the highest rank feature at the root of the tree

- Step 2: Split the training set into subsets based on their values which meet the conditions for each subset 
- Step 3: Repeat step 1 and 2 until there are terminal nodes on each edge of the decision tree

\subsection{Chapter Three Conclusion}

There are two major types of machine learning. First is the supervised or predictive learning while second is the unsupervised or descriptive learning. The machine learning process of curbing vibrations involve reducing the dimensions of rig data using principal component analysis and then assign a zone to each data point using decision tree classification. The two data acquisition processes are connecting to the electronic drilling recorder and connecting with the rig's operating system. K-means clustering is used to pick the centroid of the optimum zone. 


\section{Chapter Four: APPLICATION OF THE PROCESS}

This chapter explains how the machine learning tools discussed in chapter three are applied in this research to curb vibration problems downhole in real time. With the assumption that real time surface data has been rig from the EDR or the rig's operating system, this chapter will explain how each machine learning technique is applied to the data and what the expected output is.

\subsection{Summary of the Process}

Surface data received from the rig in real time is first corrected for data error issues and this correction is accounted for when the optimum zone is obtained. The new zone is called the safe zone. To obtain the optimum zone, the corrected data is run through principal component analysis in order to achieve dimension reduction. The dimension reduction is needed so that only useful data is being processed for getting the optimum zone. The reduced data is then taken through a decision tree where certain conditions have been inputted to determine what kind of vibration is taking place. It is this process that tells if more percentage of the data is in stick slip or whirling or in the optimum zone. At this point the correction factor from the error detection process is added to vibration prone zones. The new zone formed is called the safe zone. At this point, optimized drilling parameters are then generated. These parameters are presented as a range of values or exact values per time. It is expected that these values are updated either on a time basis or depth basis. In summary, the entire process when being tested would be on a reactionary mode but when fully prepared for use at the rig in real time, this process would function on a prescriptive mode. The next segments of this chapter will first look at the technical ways of defining what kind of vibration is occurring and then how the correction factor is applied to the optimum zone.

\subsection{Convention Operational Techniques for Curbing Vibration}

The drill rate that can be achieved with a specific bit is determined by the aggressiveness of its design, the weight on bit (WOB) applied, the rotations per minute (RPM) and the rock strength. An increase in WOB or RPM should naturally increase rate of penetration, ROP. If the increase is 
proportionate, the bit is efficient. However, if ROP does not increase with an increase in WOB, then something may be wrong with the cutting process. There is a specific dysfunction causing the depth of cut to be less than it should be. Could it be a problem with the bit or the hardness of the rock? The causes of inefficiency are known and for each type of bit dysfunction there are steps that can be taken immediately by the driller to improve the efficiency, ROP, bit life, and borehole quality [63]. In this research the dysfunction in focus is drill string vibrations. The conventional steps taken to improve efficiency depends on the type of vibration occurring. These steps are shown in Figure 16 for the three major kinds of vibrations.

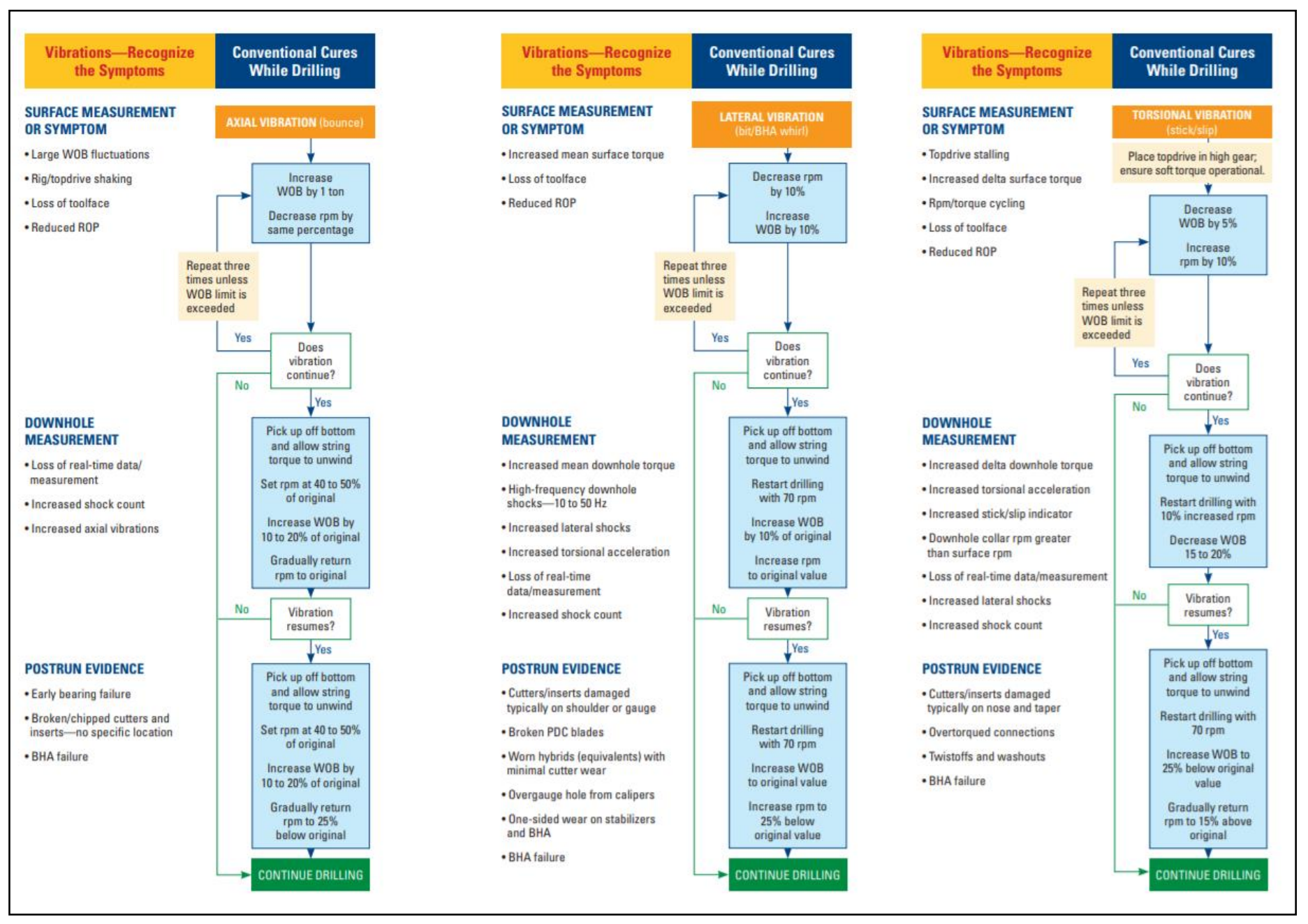

Figure 16 Drilling Optimization Mitigation Flow [64]

The chart shows symptoms at the surface when each of the vibration occurs and then shows what 
remedial steps need to be taken at the surface as well as what can be done with the drill string to reduce the effect of these vibrations. However, to create the optimum zone chart, only the mitigation steps for lateral and torsional vibrations are considered. This is because these two vibrations yield a closed loop that eventually deals with axial vibration. Since this research aims to use only surface data, the steps to curb vibration based on surface symptoms are considered alone. Figure 17 is an extract from the figure above showing based on surface symptoms just steps to reduce lateral (whirling) and torsional (stick slip) vibrations as conventionally done in the industry.

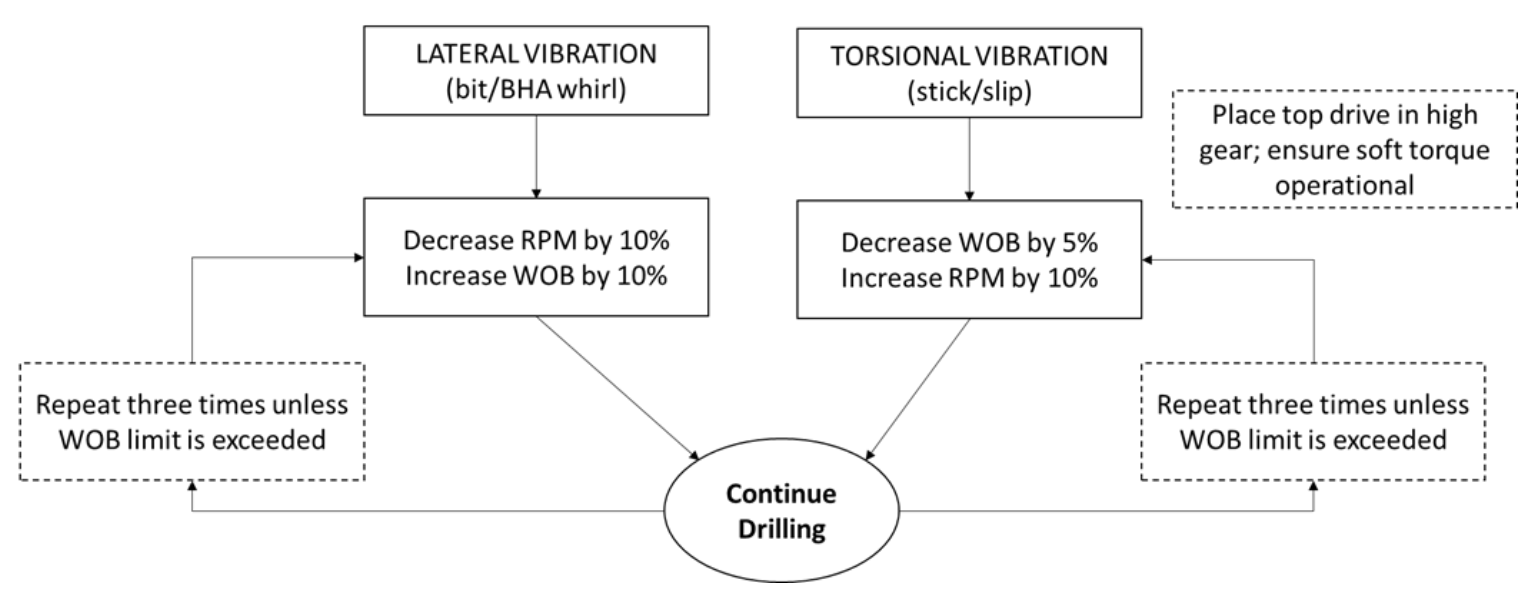

Figure 17 Conventional Ways of Curbing Lateral and Torsional Vibrations [64]

\subsection{The Optimum Zone}

The optimum zone is an envelope created when the mitigation steps for lateral and torsional vibrations are inverted and merged. The idea is that the conventional ways of curbing vibration can be reversed to give the limits of WOB and RPM as drilling occurs. Those limits are values where vibration will surely occur. Since the driller is always faced with the dilemma of inducing stick slip when trying to reduce whirling or inducing whirling when trying to reduce stick slip, the limits based on whirling and stick slip can be merged to reveal the envelope where the driller can choose WOB and RPM values that escape the effects of lateral and torsional vibration. This combination will yield the upper and lower limits of WOB and RPM every time the system is 
updated with new surface data. Since so many factors affect the drilling process, the optimum zone has to depend on other drilling parameters as well. This is where PCA comes in. The WOB and RPM used to create the optimum zone is first run through PCA with other drilling parameters to remove insignificant data. The new variables will depend on other drilling parameters because when they all input into the PCA process they are intertwined before dimension reduction occurs. Let's assume that the drilling parameters inputted into PCA are WOB, RPM, MSE, TOR, ROP or any other drilling parameter desired to have an impact on the optimum zone. If represent their values by $x_{1}, x_{2}, \ldots, x_{\mathrm{k}}$ :

From $k$ original variables: $x_{1}, x_{2}, \ldots, x_{\mathrm{k}}$ : PCA aims to produce $k$ new variables: $y_{1}, y_{2}, \ldots, y_{\mathrm{k}}$ : where

$$
\begin{gathered}
y_{1}=a_{11} x_{1}+a_{12} x_{2}+\cdots+a_{1 k} x_{k} \\
y_{2}=a_{21} x_{1}+a_{22} x_{2}+\cdots+a_{2 k} x_{k} \\
\cdots \\
y_{1}=a_{11} x_{1}+a_{12} x_{2}+\cdots+a_{1 k} x_{k}
\end{gathered}
$$

$y_{\mathrm{k}}$ 's are uncorrelated (orthogonal)

$y_{1}$ explains as much as possible of original variance in data set

$y_{2}$ explains as much as possible of remaining variance

$\left\{a_{11}, a_{12}, \ldots, a_{1 \mathrm{k}}\right\}$ is 1 st Eigenvector, $\lambda_{1}$

$\left\{a_{21}, a_{22}, \ldots, a_{2 \mathrm{k}}\right\}$ is $2 \mathrm{nd}$ Eigenvector, $\lambda_{2}$

Figure 18 below simply refreshes the understanding of how principal components relate to each other in PCA. 

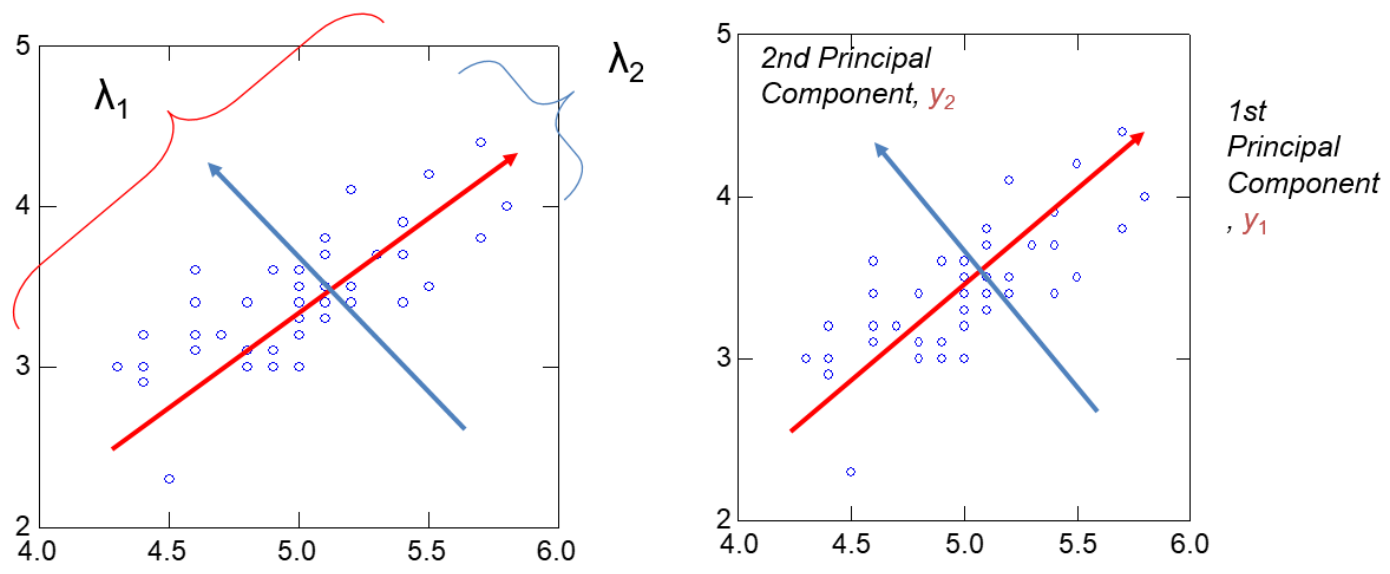

Figure 18 Illustration of Eigenvectors on a PCA Chart

$\lambda_{1} \& \lambda_{2}$ are the eigenvectors of the correlation/covariance matrix and $\lambda_{1} \& \lambda_{2}$ are the coefficients of the principal components. If $y_{1}$ and $y_{2}$ explains $99 \%$ of original data, $\left\{a_{31}, a_{32}, \ldots, a_{3 \mathrm{k}}\right\}$ up to $\left\{a_{\mathrm{k} 1}, a_{\mathrm{k} 2}, \ldots, a_{\mathrm{kk}}\right\}$ are equated to zero. Therefore

$$
\begin{gathered}
y_{1}=a_{11} x_{1}+a_{12} x_{2}+\cdots+a_{1 k} x_{k} \\
y_{2}=a_{21} x_{1}+a_{22} x_{2}+\cdots+a_{2 k} x_{k} \\
y_{3}=a_{31} x_{1}+a_{32} x_{2}+\cdots+a_{3 k} x_{k} \\
y_{4}=a_{41} x_{1}+a_{42} x_{2}+\cdots+a_{4 k} x_{k} \\
y_{5}=a_{51} x_{1}+a_{52} x_{2}+\cdots+a_{5 k} x_{k} \\
y_{k}=a_{k 1} x_{1}+a_{k 2} x_{2}+\cdots+a_{k k} x_{k} \\
\quad \text { becomes } \\
y_{1}=a_{11} x_{1}+a_{12} x_{2}+\cdots+a_{1 k} x_{k} \\
y_{2}=a_{21} x_{1}+a_{22} x_{2}+\cdots+a_{2 k} x_{k} \\
y_{3}=(0) x_{1}+(0) x_{2}+\cdots+(0) x_{k} \\
y_{4}=(0) x_{1}+(0) x_{2}+\cdots+(0) x_{k} \\
y_{5}=(0) x_{1}+(0) x_{2}+\cdots+(0) x_{k} \\
y_{k}=(0) x_{1}+(0) x_{2}+\cdots+(0) x_{k}
\end{gathered}
$$


Based on the new values of $y_{3} \ldots y_{\mathrm{k}}$, inverse PCA is performed to produce new set of $x_{1}, x_{2}, \ldots, x_{\mathrm{k}}$. At this point, the reduction has already happened. The upper and lower limits of WOB and RPM can now be determined with the new WOB and the new RPM values derived from the PCA process. This is done by reversing the conventional conditions for curbing vibrations based on surface symptoms. Figure 19 below shows that transformation. The optimum zone becomes space in the middle created when these upper and lower limits are intersected. This means when the WOB and RPM derived by the PCA are run into this system, the upper limit of WOB is gotten by increasing the average WOB value by $5 \%$ three times while the upper limit of RPM is gotten by increasing the average RPM value by $10 \%$ three times. Similarly, the lower limit of WOB is obtained by decreasing the average WOB value by $10 \%$ three times while the lower limit of RPM is obtained by decreasing the average RPM value by $10 \%$ three times.

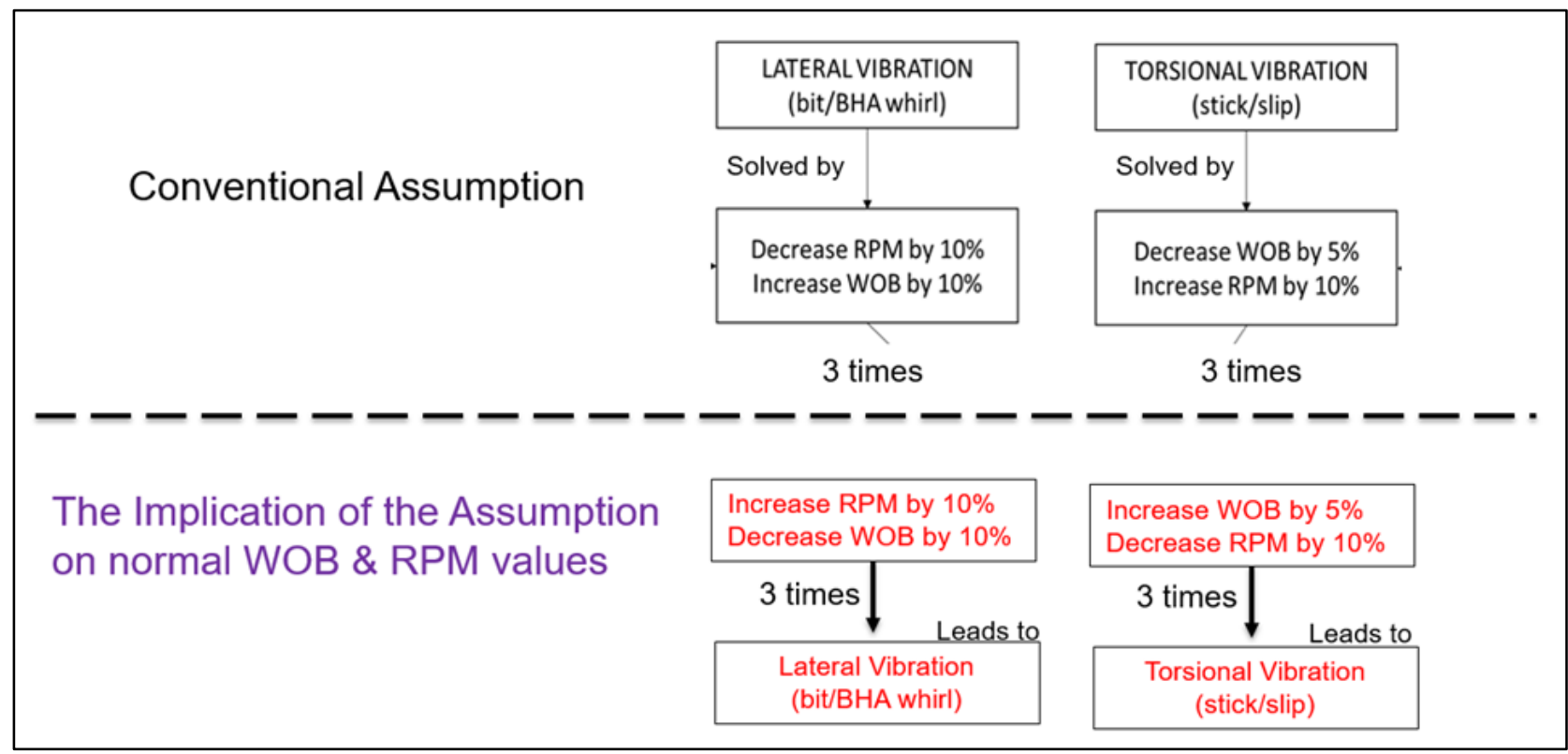

Figure 19 Obtaining Upper and Lower Limits of WOB and RPM

Increasing RPM by $10 \%$ three times means new $R P M=(1.1)^{3}($ Original $R P M)=1.331$ (Original $\left.R P M\right)$

Decreasing WOB by $10 \%$ three times means new $W O B=(0.9)^{3}($ Original $W O B)=0.729($ Original $W O B)$ Increasing WOB by $5 \%$ three times means 


$$
\text { new } W O B=(1.05)^{3}(\text { Original } W O B)=1.157625(\text { Original } W O B)
$$

Decreasing RPM by $10 \%$ three times means

$$
\text { new } R P M=(0.9)^{3}(\text { Original } R P M)=0.729(\text { Original } R P M)
$$

\subsection{Generic Classification Tree to Obtain the Optimum Zone}

The optimum zone is clearly seen when a plot of the new values of WOB and RPM is used to show the upper and lower limits of WOB and RPM as given in Figure 20.

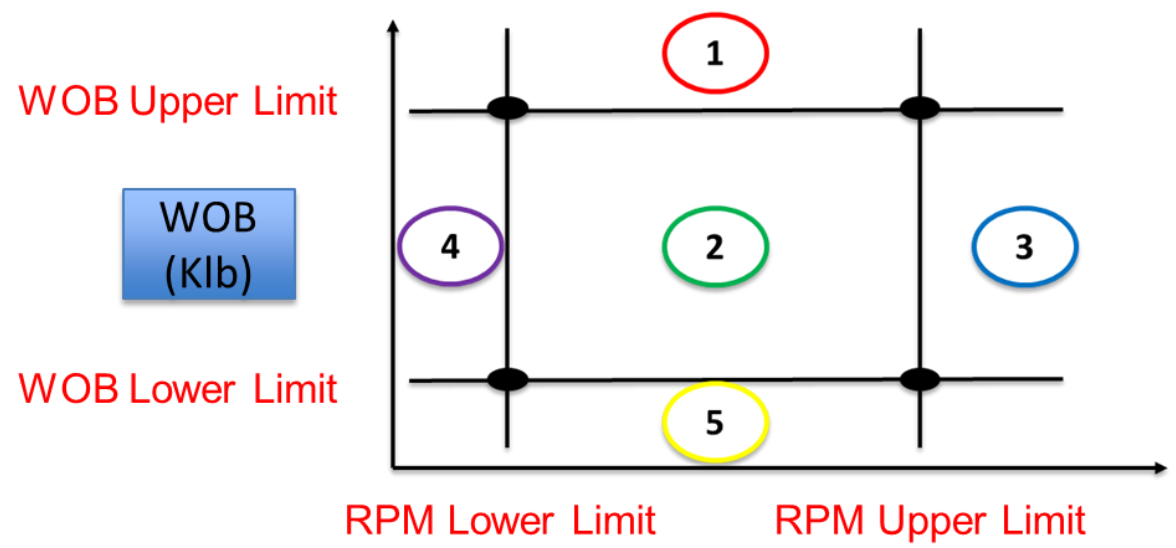

\section{RPM}

Figure 20 Basic Optimum Zone Chart

- Zone 1 is the Stick Slip Zone

- Zone 4 is the Low ROP Zone

- Zone 5 is the Forward Whirling Zone

- Zone 3 is the Backward Whirling Zone

- Zone 2 is the Optimum Zone

- WOB Upper Limit $=1.157625 *$ Average WOB; this forms the Stick Slip line

- WOB Lower Limit $=0.729 *$ Average WOB; this forms the forward whirling line

- RPM Upper Limit $=1.331 *$ Average RPM; this forms the backward whirling line

- RPM Lower Limit $=0.729 *$ Average RPM; this forms the Low ROP line

With this knowledge, a decision tree can be formed based on the fact that any data point above the 
stick slip line is on the stick slip zone and would most likely be experiencing stick slip, any data point behind the low ROP line is in the low ROP zone and would be experiencing less efficient drilling, any data point ahead of the backward whirling line would be in the backward whirling zone and would be experiencing backward whirling and finally any data point below the forward whirling line would be in the forward whirling zone and most likely be experiencing forward whirling. Based on this information, a decision tree can be developed. The figure below is the decision tree formed based on Figure 20 above. The concept is that for every 3 minutes or 3 feet interval, the optimum zone is update with new WOB and RPM values which have been processed by PCA. The structure designed in Figure 21 is formed based on the decision tree. All the data points will belong to one of the zones. The driller can now see how much of the data points are in stick slip or whirling. Based on the arrangement, the driller can either select the readings of the data points in the optimum zone or ask the system to generate a range of data points that are in the optimum zone. However, if there is a significant change in drilling parameters, the optimum zone will shift its location and new safe ranges would have to be generated. 


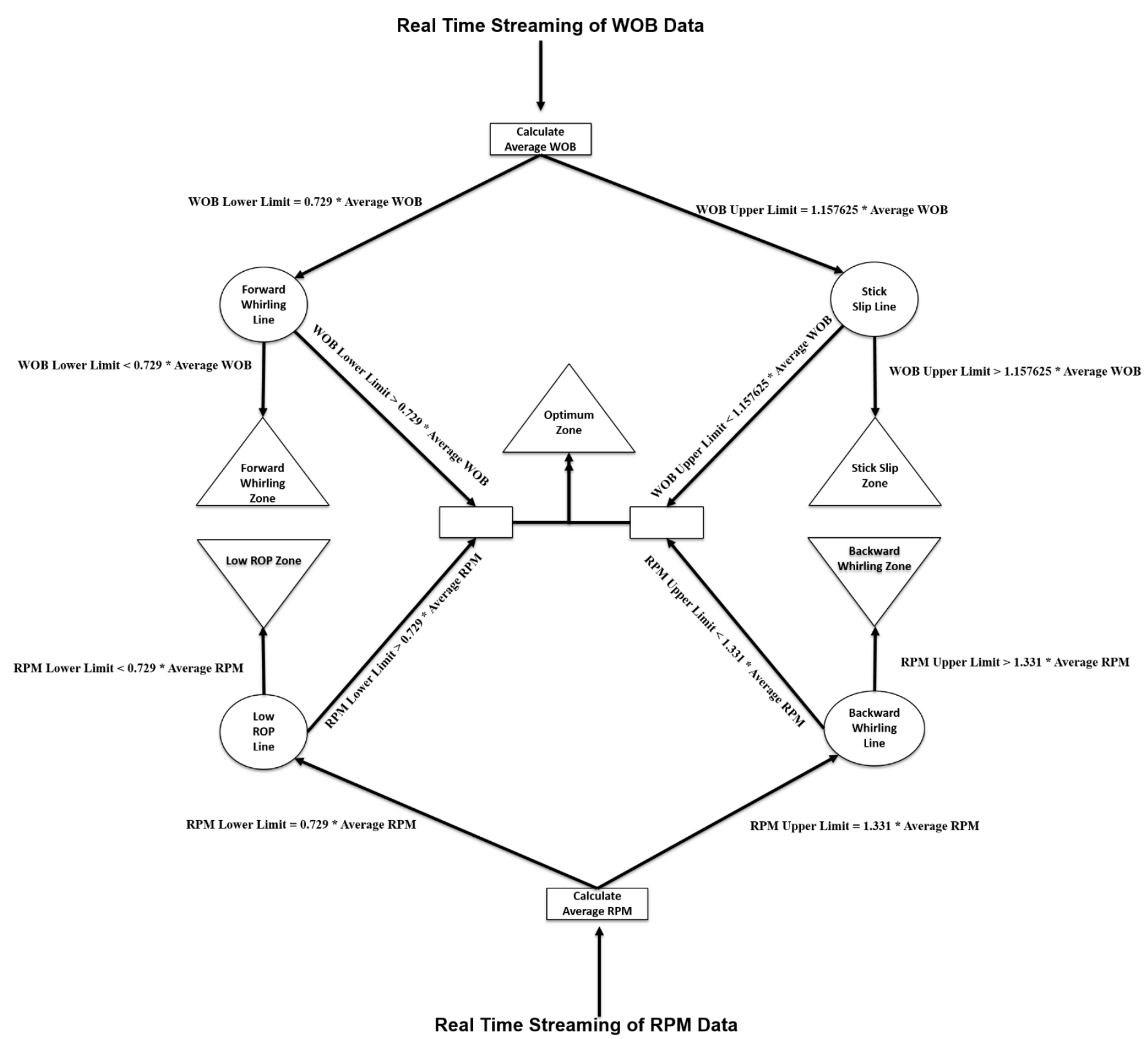

Figure 21 Decision Tree Classification to Obtain Optimum Zone Chart for Reducing Drill String Vibrations

\subsection{The Safe Zone}

The concept of the safe zone is account for the risk of having data points in the optimum zone actually be in vibration prone zones. This risk could affect the optimum zone by including some vibration prone zones in the optimum zone. This process will take note of the risk of having vibration occurring in the optimum zone. For the stick slip zone, the safety factor derived would 
be subtracted from the upper limit of the WOB, while for the forward whirling zone, the corresponding safety factor derived would be added to the lower limit of WOB. For the backward whirling zone, the corresponding the safety factor would be subtracted from the upper limit of RPM. The methodology used to derive the safety factor is quantitative risk analysis.

\subsubsection{Quantitative Risk Analysis (QRA)}

QRA has been used widely in the construction industries and has also been used in casing design and well planning by the oil and gas industries. The QRA approach considers the uncertainty of each input variable and provides comprehensive statistical properties of WOB, RPM, ROP, MSE and other drilling parameters. The perimeters needed for quantitatively calculating the risks are discussed below.

Mean value, $\mathrm{m}$, is the expected value or the weighted average.

$$
m=\frac{\sum x}{N}
$$

Standard deviation, s, is a measure of dispersion or variability. Standard deviation measures the closeness of each random variable to the mean value [65]. It is given as

$$
s=\sqrt{\frac{\sum\left(x_{i}-m\right)^{2}}{N}}
$$

Coefficient of Variance (COV) evaluates the distribution of the standard deviation over the mean value [65]. The data is more uncertain as the COV goes higher.

$$
\operatorname{COV}=\frac{s}{m}
$$

To calculate the risk of data points in the optimum zone fall into the vibration prone zones, there is a need to first determine the means and standard deviations of the stick slip zone $\left(\mathrm{M}_{\mathrm{SS}}\right.$ and $\left.\mathrm{S}_{\mathrm{SS}}\right)$, the backward whirling zone $\left(\mathrm{M}_{\mathrm{BW}}\right.$ and $\left.\mathrm{S}_{\mathrm{BW}}\right)$, the forward whirling zone $\left(\mathrm{M}_{\mathrm{FW}}\right.$ and $\left.\mathrm{S}_{\mathrm{FW}}\right)$ and the optimum zone (Mop and $\left.\mathrm{S}_{\mathrm{OP}}\right)$.

- For normally distributed stick slip and optimum zone data, the margin between the two 
probability density functions (PDFs) has a mean margin of

$$
M_{S O}=M_{S S}-M_{O P}
$$

And standard deviation margin of

$$
S_{S O}=\sqrt{\left(S_{S S}\right)^{2}+\left(S_{O P}\right)^{2}}
$$

The risk of having optimum zone data points in stick slip zone $=R_{S O}=\left(\frac{M_{S O}}{S_{S O}}\right)$;

In order to give the driller some more space to change parameters, $20 \%$ of the risk can be allowed

Therefore, $R_{S O}=80 \%\left(\frac{M_{S O}}{S_{S O}}\right)$; this is the safety factor for the stick slip zone.

- For normally distributed optimum zone and forward whirling data, the margin between the two probability density functions (PDEs) has a mean margin of

$$
M_{O F}=M_{O P}-M_{F W}
$$

And standard deviation margin of

$$
S_{O F}=\sqrt{\left(S_{O P}\right)^{2}+\left(S_{F W}\right)^{2}}
$$

The risk of having forward whirling zone data points in optimum zone $=R_{O F}=\left(\frac{M_{O F}}{S_{O F}}\right)$;

In order to give the driller some more space to change parameters, can take $20 \%$ of the risk can be allowed

Therefore, $R_{O F}=80 \%\left(\frac{M_{O F}}{S_{O F}}\right)$; this is the safety factor for the forward whirling zone.

- For normally distributed backward whirling and optimum zone data, the margin between the two probability density functions (PDEs) has a mean margin of

$$
M_{B O}=M_{B W}-M_{O P}
$$

And standard deviation margin of

$$
S_{B O}=\sqrt{\left(S_{B W}\right)^{2}+\left(S_{O P}\right)^{2}}
$$


The risk of having optimum zone data points in backward whirling zone $=R_{B O}=\left(\frac{M_{B O}}{S_{B O}}\right)$; In order to give the driller some more space to change parameters, $20 \%$ of the risk can be allowed

Therefore, $R_{B O}=80 \%\left(\frac{M_{B O}}{S_{B O}}\right)$; this is the safety factor for the backward whirling zone.

Figure 22 below shows how the optimum zone would look like after QRA is applied. The concept is that risk is determined obtaining the optimum zone envelope without using PCA but the safety factor is applied on the optimum zone chart after PCA has been applied to the drilling parameters inputted into the system.

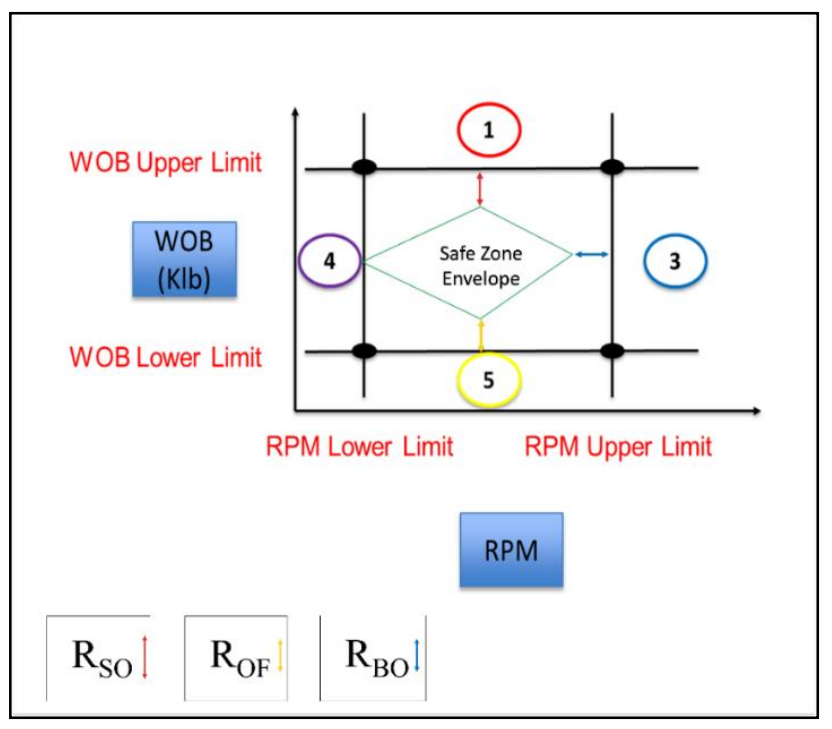

\section{Figure 22 Optimum Zone Chart Showing Safe Zone}

The concept of the safe zone is still subject to data validation and field testing. However, it helps the driller not to make border line decisions which are too close to vibration zones. For regions which are prone to high vibration problems, $20 \%$ allowance may not be removed from the risk of having optimum zone data points in the vibration zones. In these zones, $\mathrm{R}_{\mathrm{SO}}, \mathrm{R}_{\mathrm{OF}}$, and $\mathrm{R}_{\mathrm{BO}}$ can be removed from the optimum zone in full in order to actualize full safety from vibration problems while drilling. 


\subsection{Chapter Four Conclusion}

The conventional ways of mitigating the three modes of vibrations were reviewed but only the mitigation steps for lateral and torsional vibrations were considered. The idea is that the conventional ways of curbing vibrations were reversed to get the upper and lower limits of WOB

and RPM. Multiple drilling parameters from the rig were first run through PCA before classification into various vibration zones was done based on the WOB and RPM values. 


\section{Chapter Five: ANALYSIS OF RESULTS}

This chapter looks at the result of each step in the optimization process. In the first phase of the results, the optimization is tested on historical surface data and then compared with downhole data recorded results. The second phase of the results is an attempt to simulate streaming of real-time data as fast as an actual field scenario. The severity of the vibration measured tells if the optimization is successful or not.

\subsection{The Types of Results Expected}

It is assumed that the data has been collated from the rig into the built machine learning system of this research. The results presented here are the outcome of each step in the machine learning process. The first set of results would be from the principal component analysis (PCA) done on all the field data fed to the system. The principal components and their respective percentage of significance would be derived. The principal components that make up at least $98 \%$ of the data would be chosen while the other principal components are zeroed out before an inverse PCA is performed to obtain the leaner original data. Based on the decision tree classification, each data point is then classified into one of the five zones in the WOB and RPM plot. The quantitative risk analysis results are shown and then applied to the optimum zone chart to produce the safe zone plot.

\subsection{Post Analysis with Field Data}

The data is drawn from a well in continental United States. Plotting bit depth, "BitDepth" and measured depth, "MeasuredDepth" versus time gives the trend in Figure 23. This analysis would be done on one stand of drill string pipe. For this well, a depth of 3.5 feet is drilled in 3.4 minutes. For this post analysis, the entire data for the region for the selected stand would be analysed for vibration issues and classified into the five zones. The stand chosen is one with no obvious issues. The visible signs of problems with the data from a stand are inequalities between the bid depth and the measured depth. It is the bit depth that is very essential; it tells that the drill string is moving 
into the formation and not just rotating at a spot. Any stand that has a constant depth for a while is an indication of stoppage in drilling or pause in drilling forward. Figure 24 below shows the plot of bit depth, measured depth versus time for the entire well.

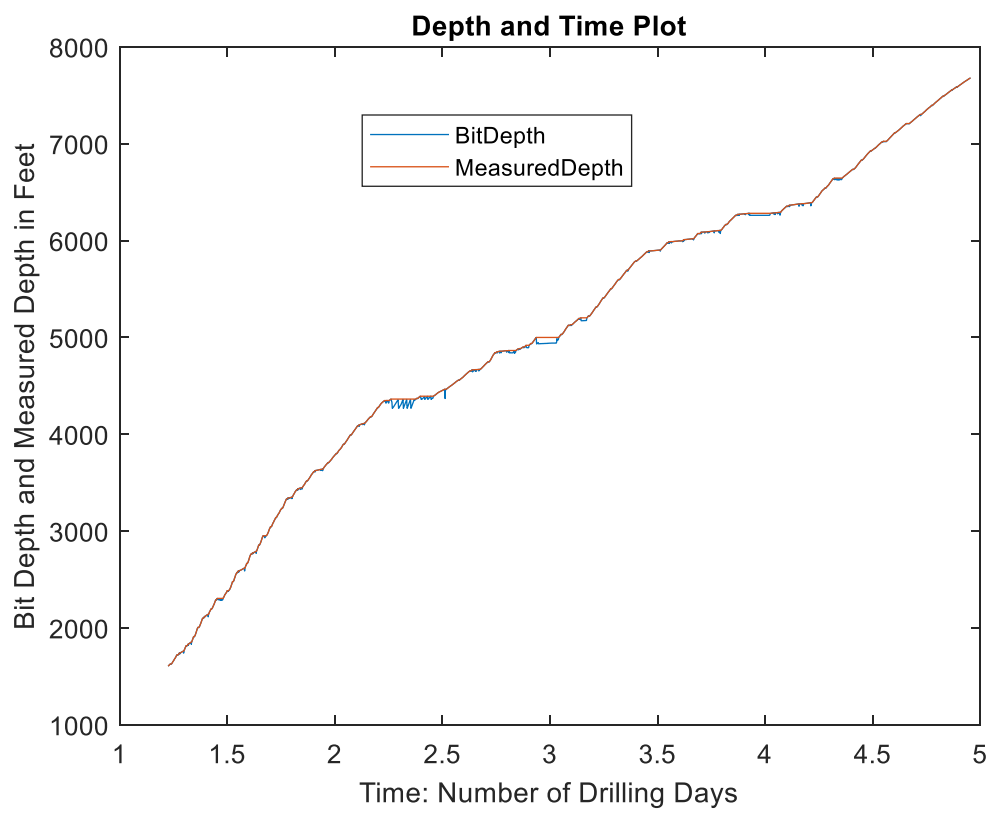

Figure 23 Depth Versus Time for the Well used for Post Analysis

In Figure 24 below, the stand to be studied was run for 25 minutes without any stopping; the data would be reliable and feasible updates to the optimum zone can be done at 3.50 minutes for each update. The optimum zone would be compared with downhole data provided to see if the data points for optimum/safe zone drilling correlate with the data points for good drilling based on actual downhole events. 


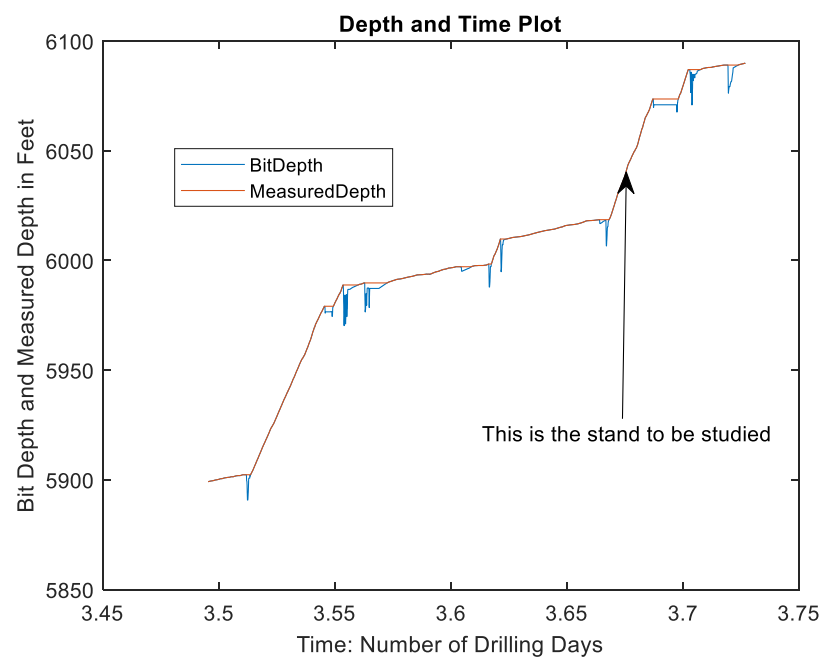

Figure 24 Identifying the Stand for the Post Well Analysis

\subsection{Principal Component Analysis (PCA) Results}

The first data received for the first 3.50 minutes has 9 parameters. These are ROP, WOB, RPM, bit depth, measured depth, time, stick slip indicator, axial vibration indicator and lateral vibration indicator. At the end of the analysis, the first three principal components were found to make up at least $98 \%$ of the original data.

Table 3 Significance Level for Principal Components in Update One

\begin{tabular}{|c|c|}
\hline Principal Component & Percent Significance of Original Data \\
\hline 1 & 90.8999 \\
\hline 2 & 6.3755 \\
\hline 3 & 2.5820 \\
\hline 4 & 0.1331 \\
\hline 5 & 0.0088 \\
\hline 6 & 0.0004 \\
\hline 7 & 0.0001 \\
\hline 8 & 0.0000 \\
\hline 9 & 0.0000 \\
\hline
\end{tabular}


In order to further show how the first principal component carries more significance, a plot between the most significant principal components can be computed as shown in the Figure 25 below. In this case, the first 3 principal components; the plot shows the bulk of the trend flowing in the first principal component axis showing further that it explains more of the original data.

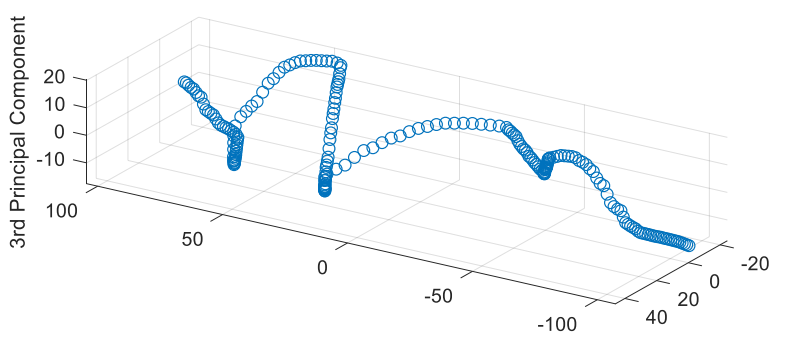

1st Principal Component

2nd Principal Component

Figure 25 3D Plot of 1st, 2nd and 3rd Principal Components for Update 1

Now a comparision of results from all 7 updates. Recall that since the data is historical data, the results are taken based on the data points in that data collation period.

Table 4 Significance Level for Principal Components for Updates One to Update Seven

\begin{tabular}{|c|c|c|c|c|c|c|c|}
\hline \multirow{2}{*}{$\begin{array}{c}\text { Principal } \\
\text { Component }\end{array}$} & \multicolumn{7}{|c|}{ Percent Significance in Original Data } \\
\cline { 2 - 8 } & $\mathbf{1}^{\text {st }}$ Update & $\mathbf{2}^{\text {nd }}$ Update & $\mathbf{3}^{\text {rd }}$ Update & $\mathbf{4}^{\text {th }}$ Update & $\mathbf{5}^{\text {th }}$ Update & $\mathbf{6}^{\text {th }}$ Update & $\mathbf{7}^{\text {th }}$ Update \\
\hline 1 & 90.8999 & 90.9354 & 92.4135 & 93.4455 & 91.5716 & 90.1617 & 85.3801 \\
\hline 2 & 6.3755 & 7.7807 & 4.7127 & 4.3283 & 7.4603 & 9.4498 & 14.1471 \\
\hline 3 & 2.5820 & 1.1043 & 2.6506 & 2.2042 & 0.8540 & 0.3086 & 0.4472 \\
\hline 4 & 0.1331 & 0.1478 & 0.2114 & 0.0163 & 0.1042 & 0.0593 & 0.0220 \\
\hline 5 & 0.0088 & 0.0313 & 0.0109 & 0.0054 & 0.0085 & 0.0199 & 0.0028 \\
\hline 6 & 0.0004 & 0.0003 & 0.0006 & 0.0003 & 0.0010 & 0.0003 & 0.0004 \\
\hline 7 & 0.0001 & 0.0002 & 0.0001 & 0.0001 & 0.0002 & 0.0003 & 0.0003 \\
\hline 9 & 0.0000 & 0.0001 & 0.0000 & 0.0000 & 0.0002 & 0.0001 & 0.0002 \\
\hline 9 & 0.0000 & 0.0000 & 0.0000 & 0.0000 & 0.0000 & 0.0000 & 0.0000 \\
\hline
\end{tabular}


In most updates, the first 3 principal components make up $98 \%$ of significance in the original data except the $6^{\text {th }}$ and $7^{\text {th }}$ updates. The figure below shows the comparison of the first 3 principal components of the second to seventh updates.
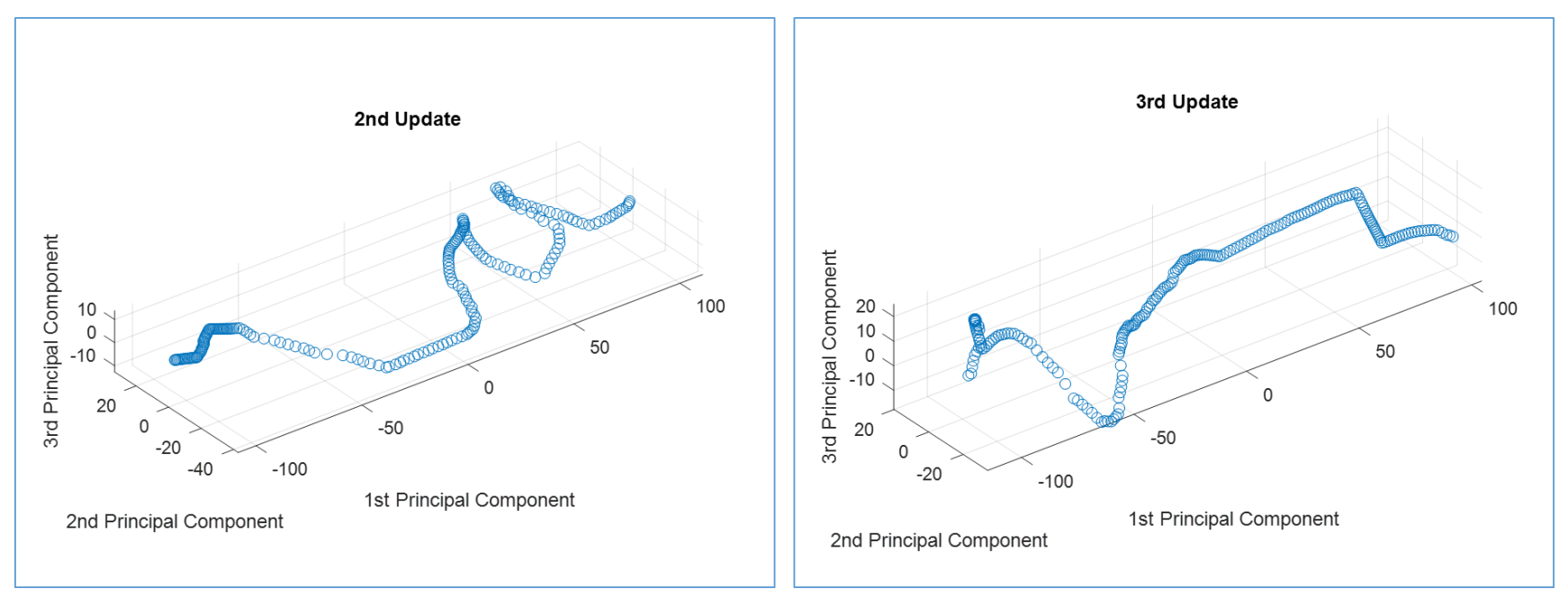

Figure $263 D$ Plot of 1st, 2nd and 3rd Principal Components for Update 2 and Update 3
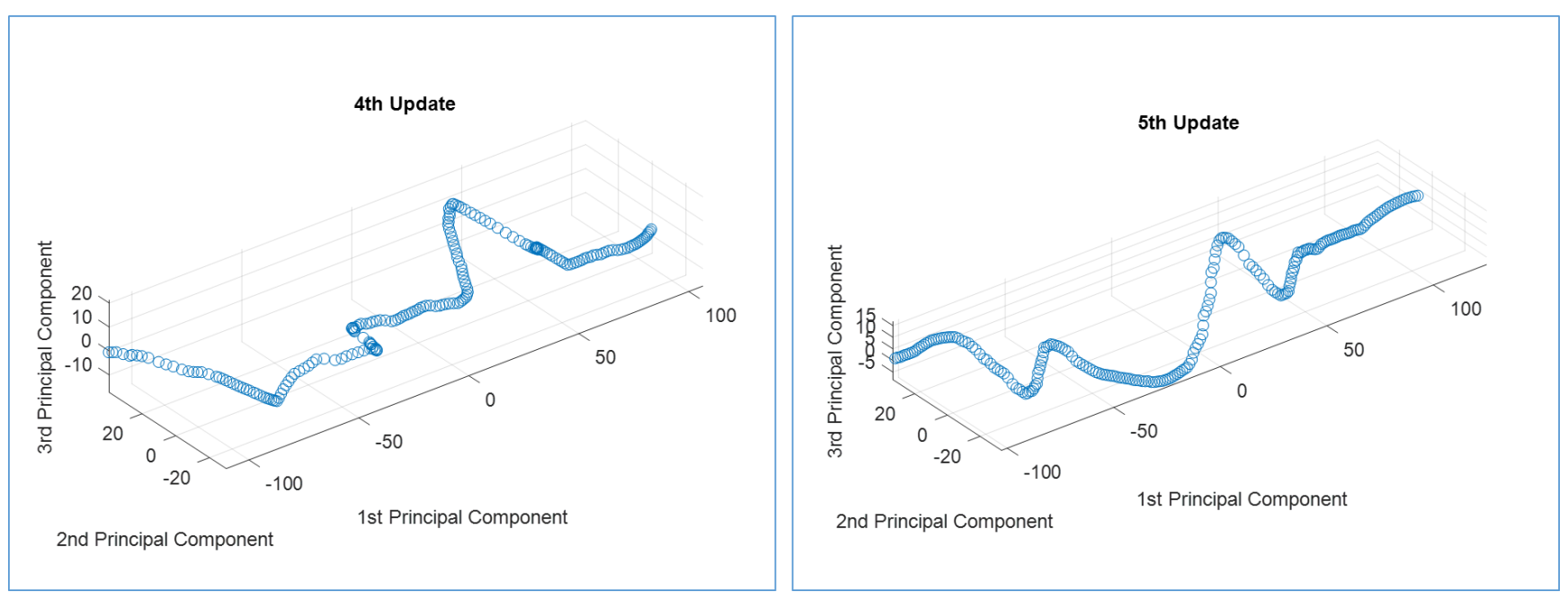

Figure 27 3D Plot of 1st, 2nd and 3rd Principal Components for Update 4 and Update 5 

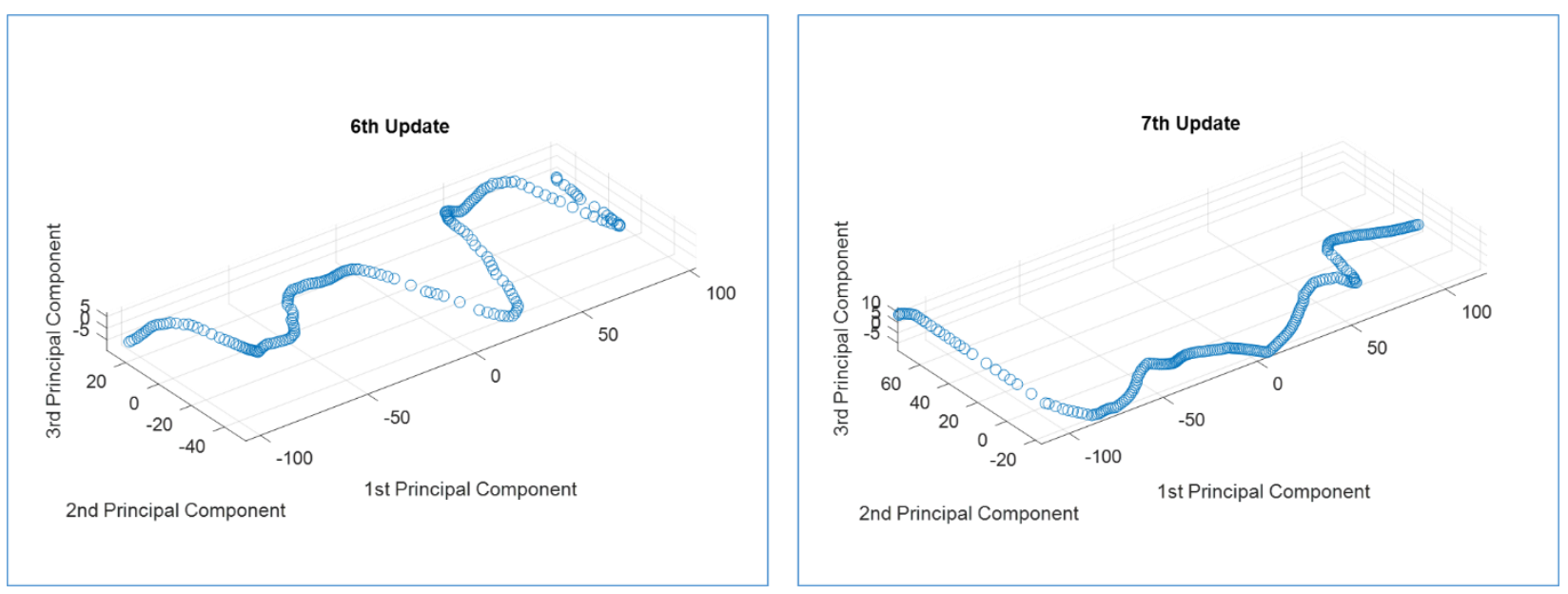

Figure 28 3D Plot of 1st, 2nd and 3rd Principal Components for Update 6 and Update 7

In the $7^{\text {th }}$ update where the first and second principal components explain the bulk of the data, the spiral trend does not move in the third principal component axis (z-axis) indicating no useful information can be found there.

\subsection{Decision Tree Results}

The data used to verify the classification tree has been processed to be divided into the five zones. The Matlab classification learner app would be used to check if the classification route used is correct and is simple enough. Figure 29 below shows the layout of the data in a WOB/RPM plot. The dataset had already been classified based on Figure 20. However, when the dataset was run through the Matlab classification learner app, the app derived the classes itself based on the patterns in the data and fixed the colours accordingly. This process was not done to define the classes, rather it was done to affirm that the classification based on Figure 20 actually led to pattern distinction in the dataset. 

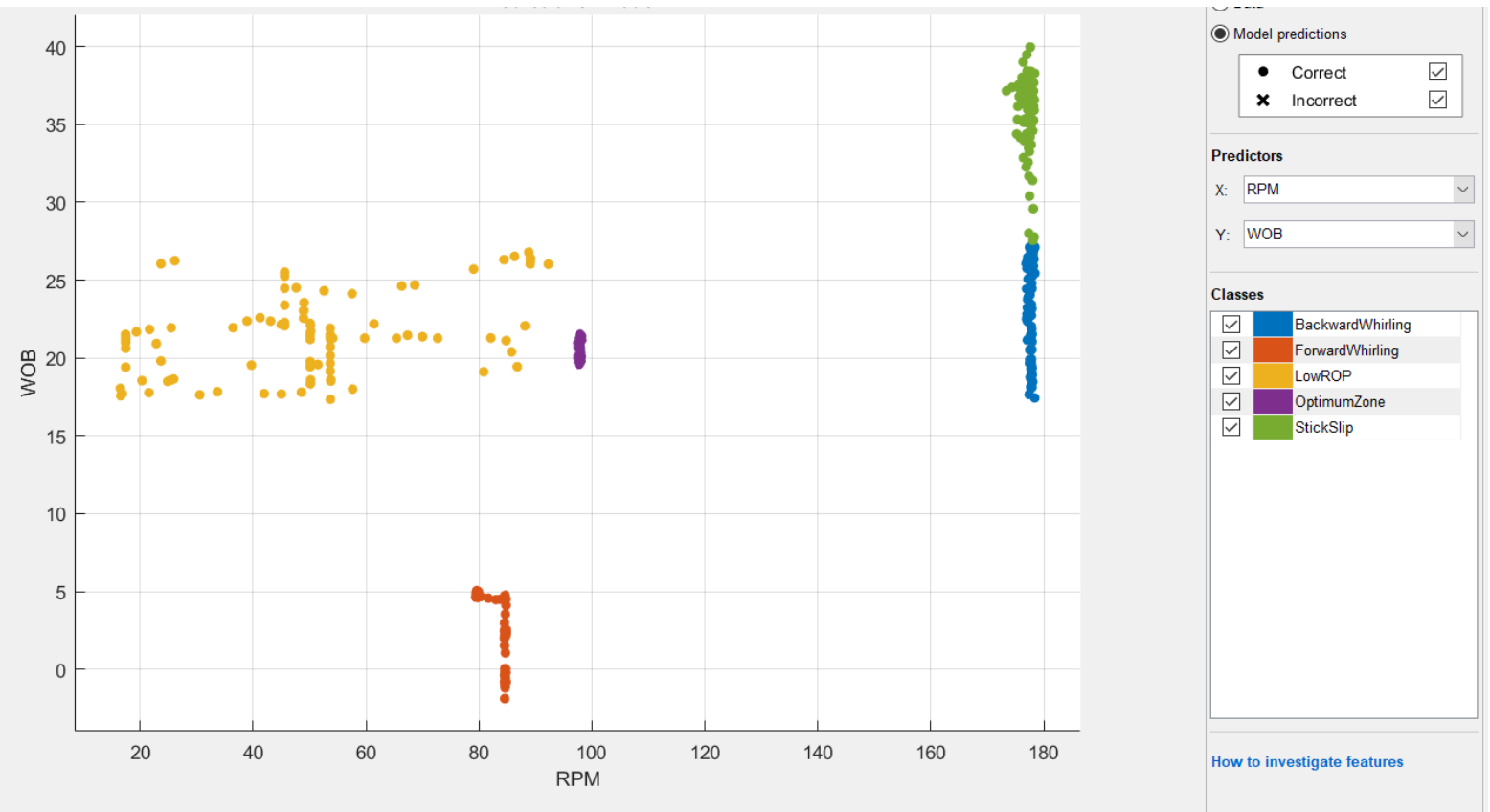

Figure 29 WOB versus RPM Plot on Matlab Classification Learner App

The color patterns show that there is a clear distinction between the five zones for this data. Next the classification tree can be generated. Figure 30 below shows the classification tree based on this data. From the data, some basic facts can be derived to create the optimum zone.

- $\quad$ RPM Lower Limit - 94.8762

- $\quad$ RPM Upper Limit - 135.78

- $\quad$ WOB Lower Limit - 11.2027

- $\quad$ WOB Upper Limit - 27.3655 


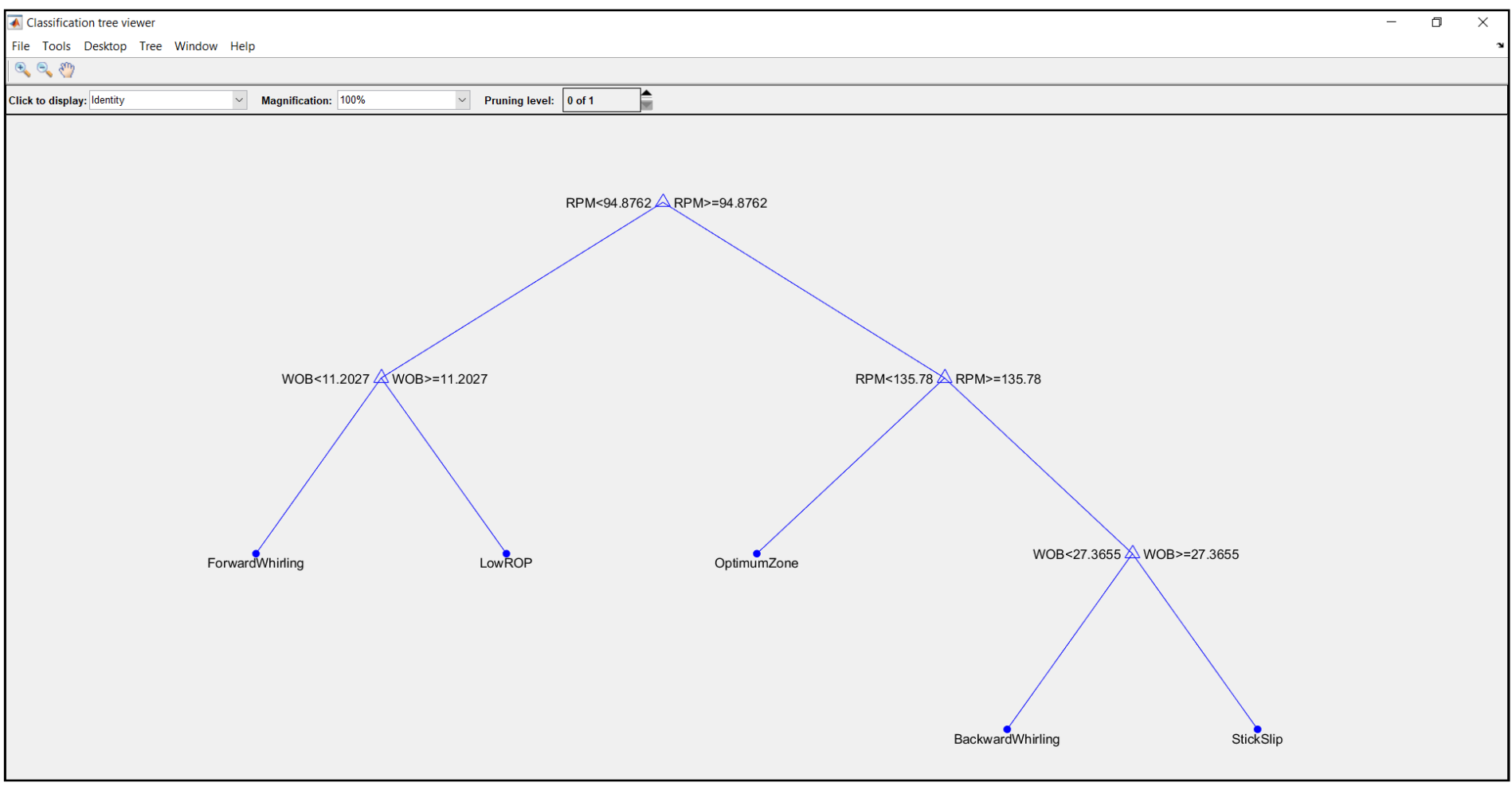

Figure 30 Decision Tree Result from Random Well Data

The figure above shows a more simplified decision tree. Now the initial generic decision classification tree can be modified to mirror the figure above since the Matlab generated decision tree is also based on the upper and lower limits WOB \& RPM. Figure 31 below shows the new generic decision classification tree to obtain data points of WOB and RPM in the optimum zone. 


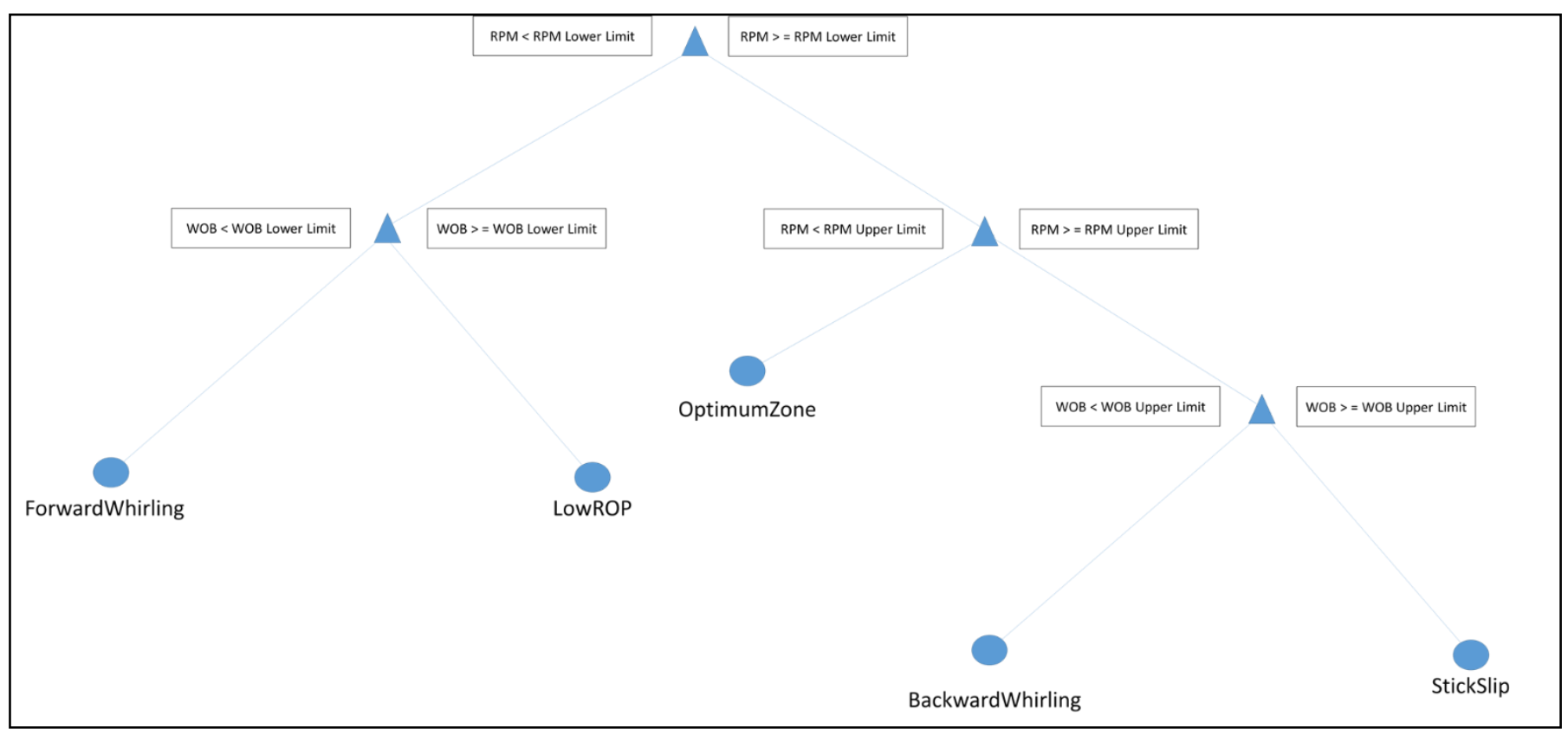

Figure 31 Revised Generic Decision Tree to Obtain Optimum Zone

\subsection{Optimum Zone Results with or without PCA}

To derive the true optimum zone for the test dataset, the optimum zone chart must be validated with recorded events downhole using vibration measurement tools downhole. The vibration measurement of concern is stick slip and lateral vibration (whirling). The procedure is that the WOB and RPM values for each data point are classified based on the downhole recording of what event happened. The optimum zone chart is then run for each updates of the test stand and compared with the corresponding downhole recording. The result is checked when using just the optimum zone and when the safe zone system is applied. Both cases are run without PCA. The PCA is applied when the recommended values of WOB and RPM values are needed.

For Figure 32 to Figure 47, good drilling refers to a combination of WOB and RPM parameters which do not meet the conditions for torsional vibration (stick-slip), axial vibration (bit-bounce) or lateral vibration (whirling). Table 5 below shows the industry accepted standard for vibration severity. 
Table 5 Vibration Severity Levels Based on Downhole Measurements [83]

\begin{tabular}{|c|c|c|c|c|c|}
\hline \multicolumn{2}{|c|}{ Lateral Acc } & \multicolumn{2}{|c|}{ Lateral RMS Acc } & \multicolumn{2}{|c|}{ Stick-Slip } \\
\hline (g's) & Severity Level & (g's) & Severity Level & $(-)$ & Severity Level \\
\hline $0-15$ & Normal & \multirow{2}{*}{$0-2.5$} & \multirow{2}{*}{ Normal } & $0-0.5$ & Low \\
\hline $15-35$ & Moderate & & & $0.5-1$ & Moderate \\
\hline $35+$ & Severe & $2.5+$ & Severe & $1+$ & Severe \\
\hline
\end{tabular}

Based on the downhole recorded data, torsional vibrations were recorded using the equation below

$$
\begin{gathered}
\text { if average } R P M>10 \\
\text { Percent Stick Slip }=\frac{100 *(\text { Max RPM }- \text { Min RPM })}{(2 * \text { Average RPM })} \\
\text { Else } \\
\text { Percent Stick Slip }=0
\end{gathered}
$$

In the case of the stand being studied, maximum stick slip recorded was $116.3153 \%$, minimum stick slip recorded was $44.1868 \%$ and the average stick slip recorded was $66.12294 \%$. The cut-off point chosen after which intolerable stick slip occurs is $50 \%(0.50)$. This is in accordance with the table 5 .

The maximum lateral vibration recorded for this stand was 3.2 RMS acceleration while the minimum lateral vibration was 2.1 RMS acceleration (RMS means root mean square). The average lateral vibration is 2.6 RMS acceleration. The cut-off point chosen after which severe lateral vibration occurs is 2.5 . This is severe lateral vibration according to the figure above. This assumption is used to classify the different scenarios occurring in update one up until update 7. Good drilling is any data point with its lateral vibration value less than 2.5 and its torsional 
vibration value less than $50 \%$

Figure 32 below shows that all the good drilling data points are on the optimum zone which is good. There are no incidences of whirling but some torsional drilling as recorded by downhole data are on the optimum zone.

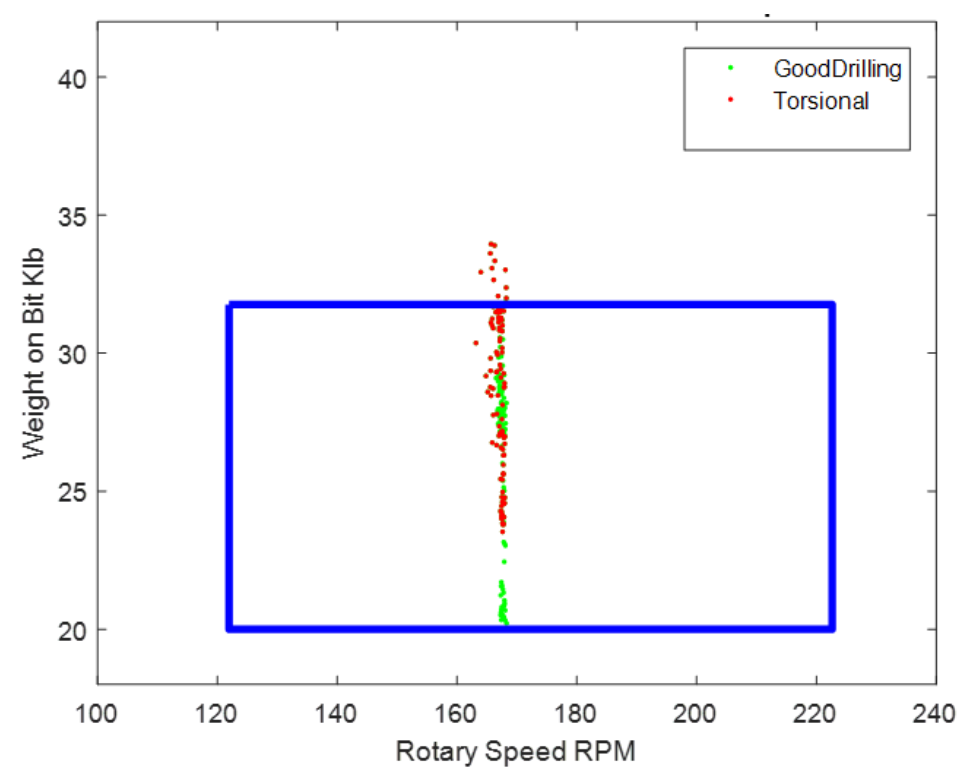

Figure 32 Classification Based on Downhole Data for Update 1

Now the safe zone can be applied on the optimum zone chart in order to exclude some of those stick slip data.

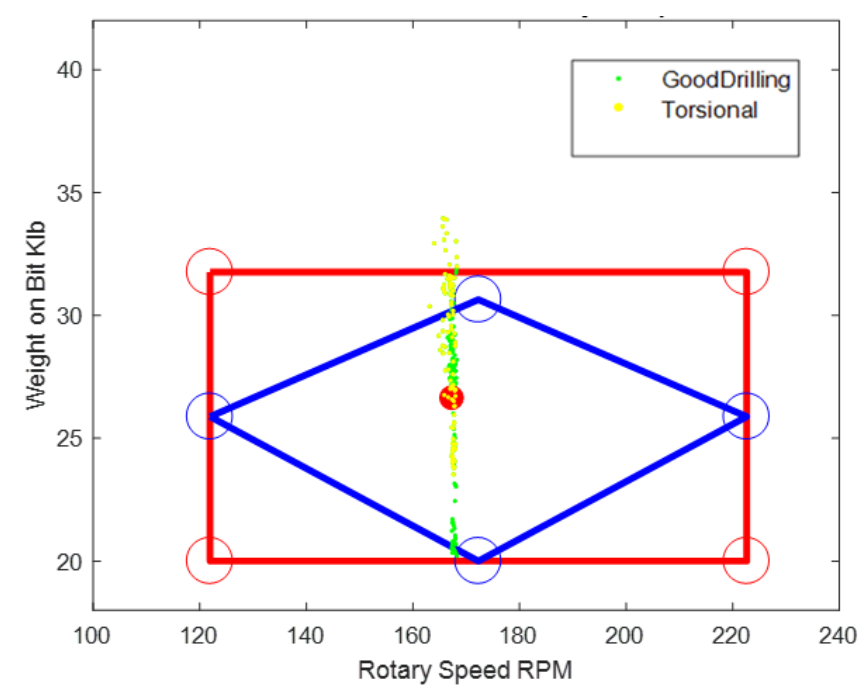

Figure 33 Classification with PCA and Safety for Update 1

Using the safe zone chart, a bit of the stick slip data has been isolated from the new optimum zone. 
However, there are still some stick slip values in the optimum zone. A test on update 2 will tell if this is a trend or not. The figures below show similar for update 2 to update 7 . Update 2 has all the data points in the optimum zone but there is a clear distinction based on downhole recorded data that there exist torsional and lateral vibrations.

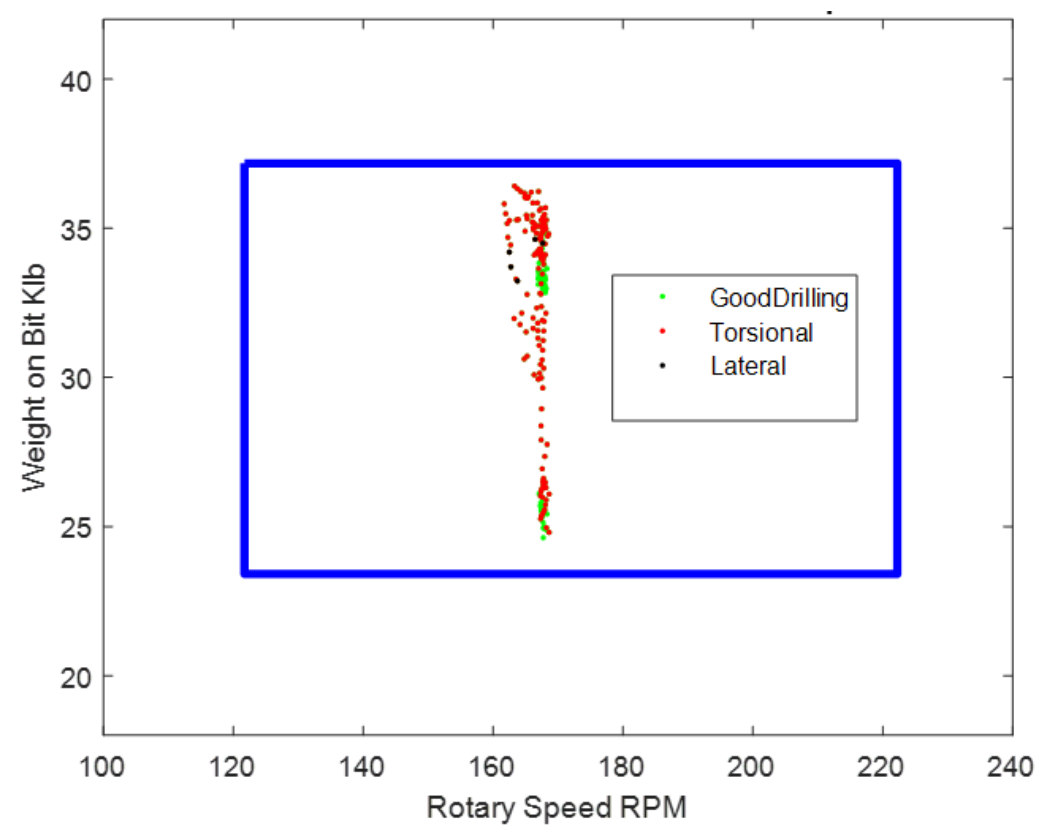

Figure 34 Classification Based on Downhole Data for Update 2

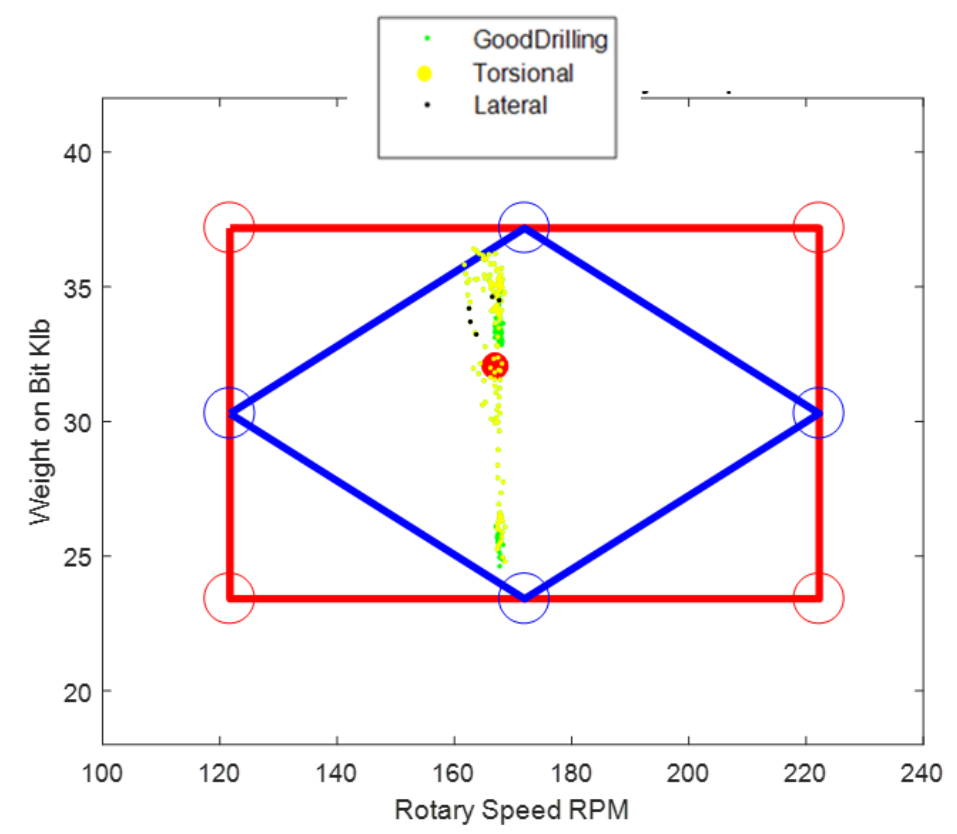

Figure 35 Classification with PCA and Safety for Update 2 
Figure 36 and Figure 37 below show a scenario where part of the torsional vibration as recorded downhole fall into the stick slip zone of the optimum zone chart. However, some stick slip data points still fall in the optimum zone.

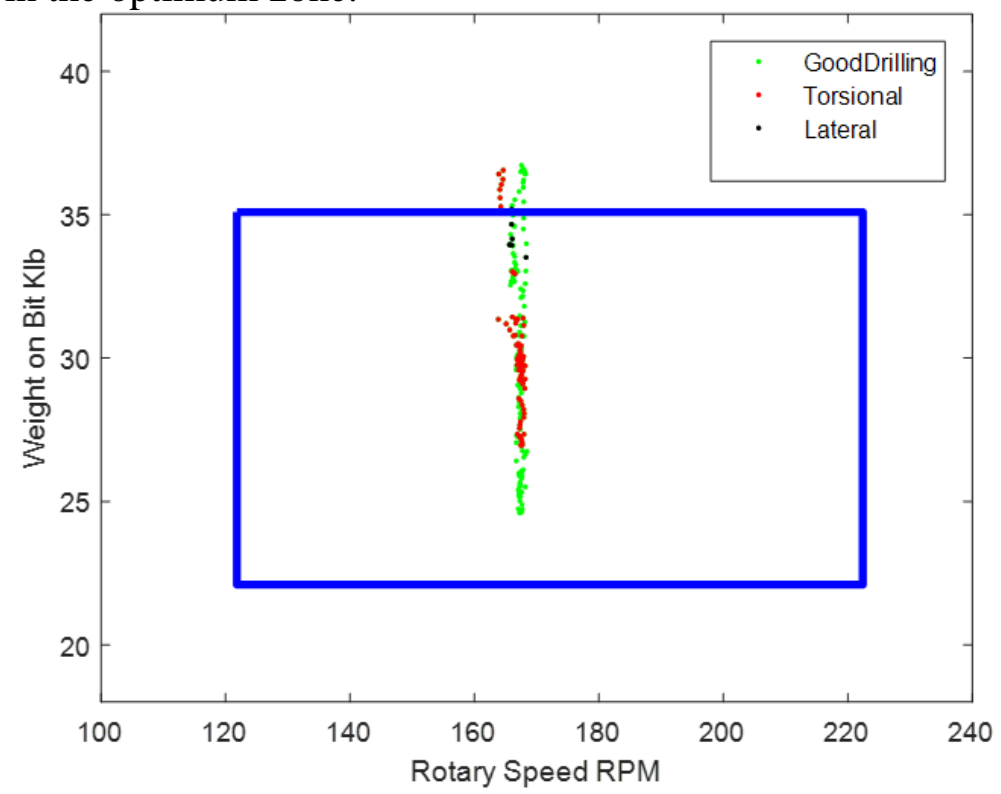

Figure 36 Classification Based on Downhole Data for Update 3

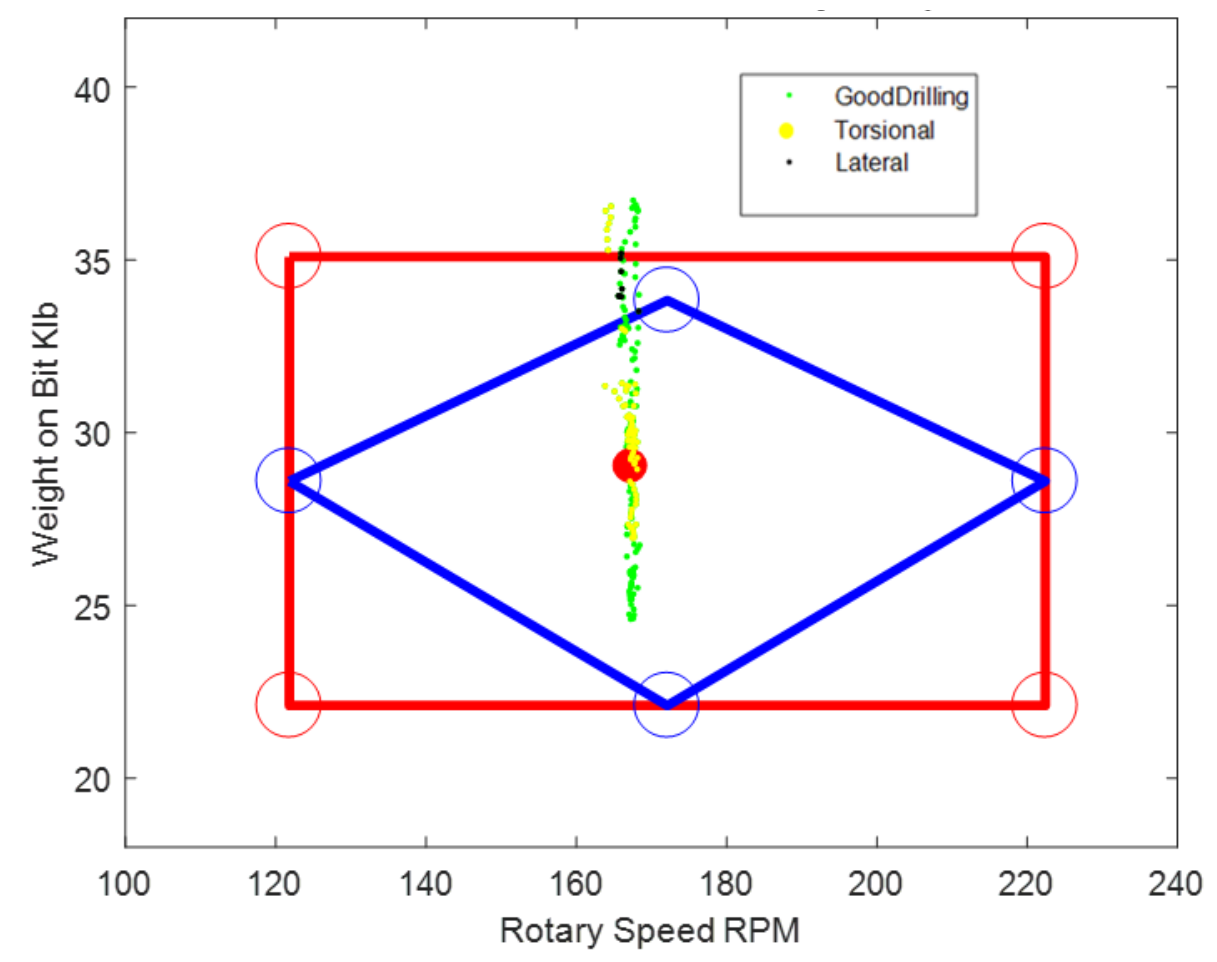

Figure 37 Classification with PCA and Safety for Update 3

Figure 38 and figure 39 below are plots for update 4. There are no good drilling data points but all the 
data fall into the optimum zone. Only torsional and lateral vibration exist according to the downhole recorded data.

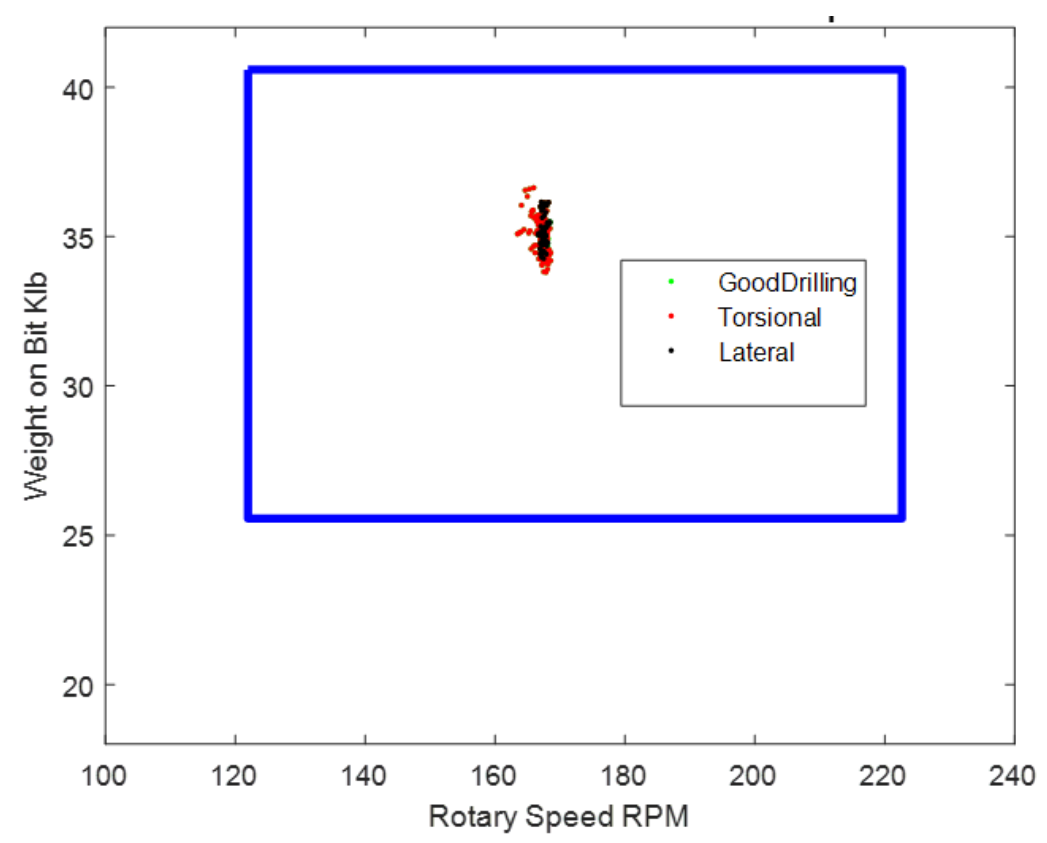

Figure 38 Classification Based on Downhole Data for Update 4

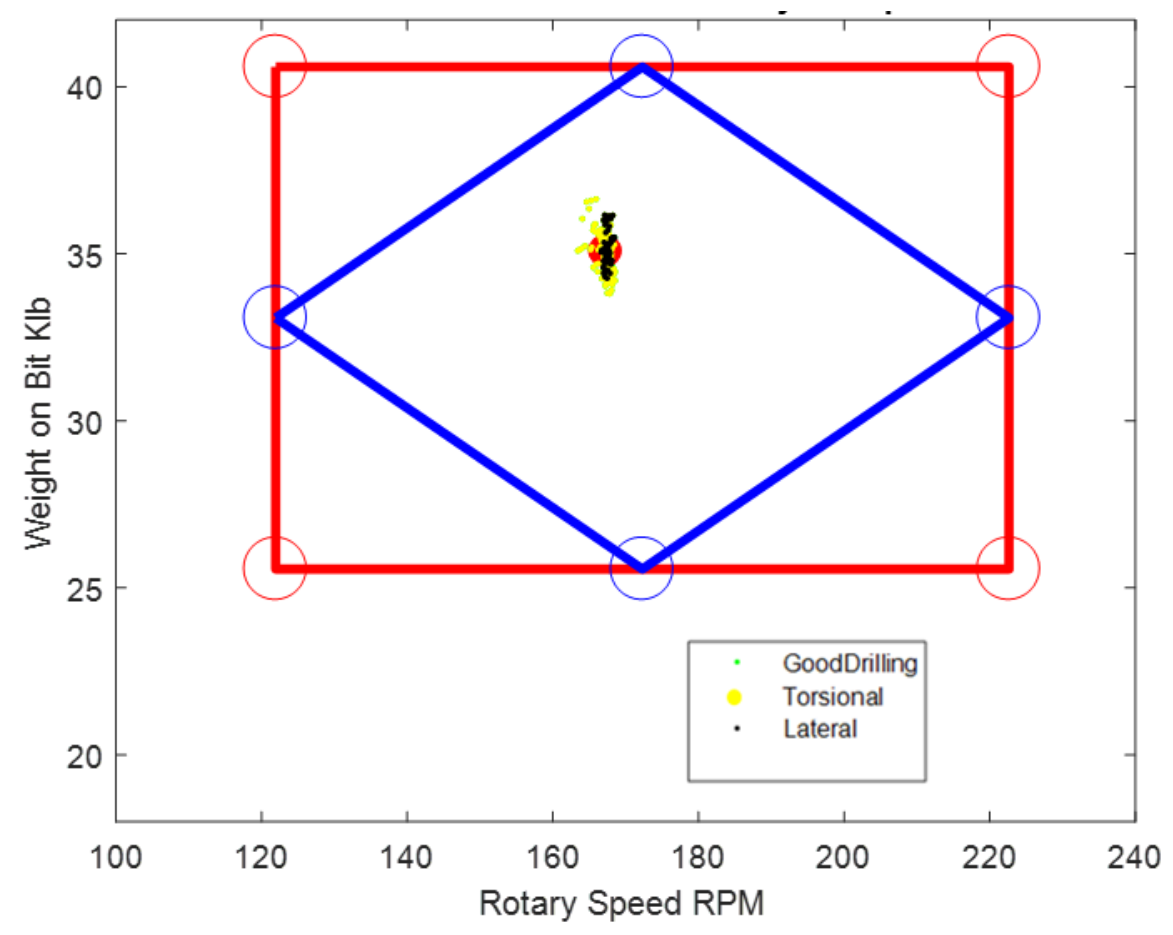

Figure 39 Classification with PCA and Safety for Update 4

For update 5 (Figure 40 and Figure 41), there is a good mix of all conditions (torsional, lateral and good 
drilling). However, all the data points lie in the optimum zone.

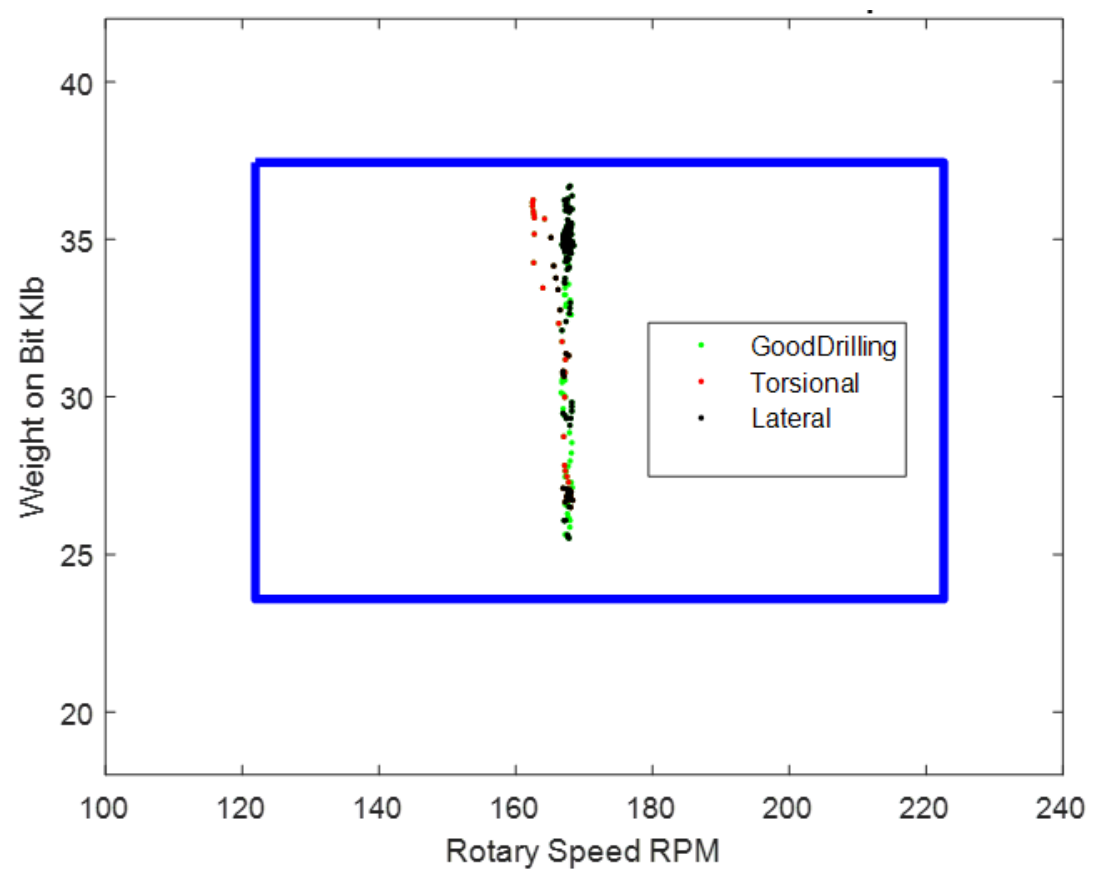

Figure 40 Classification Based on Downhole Data for Update 5

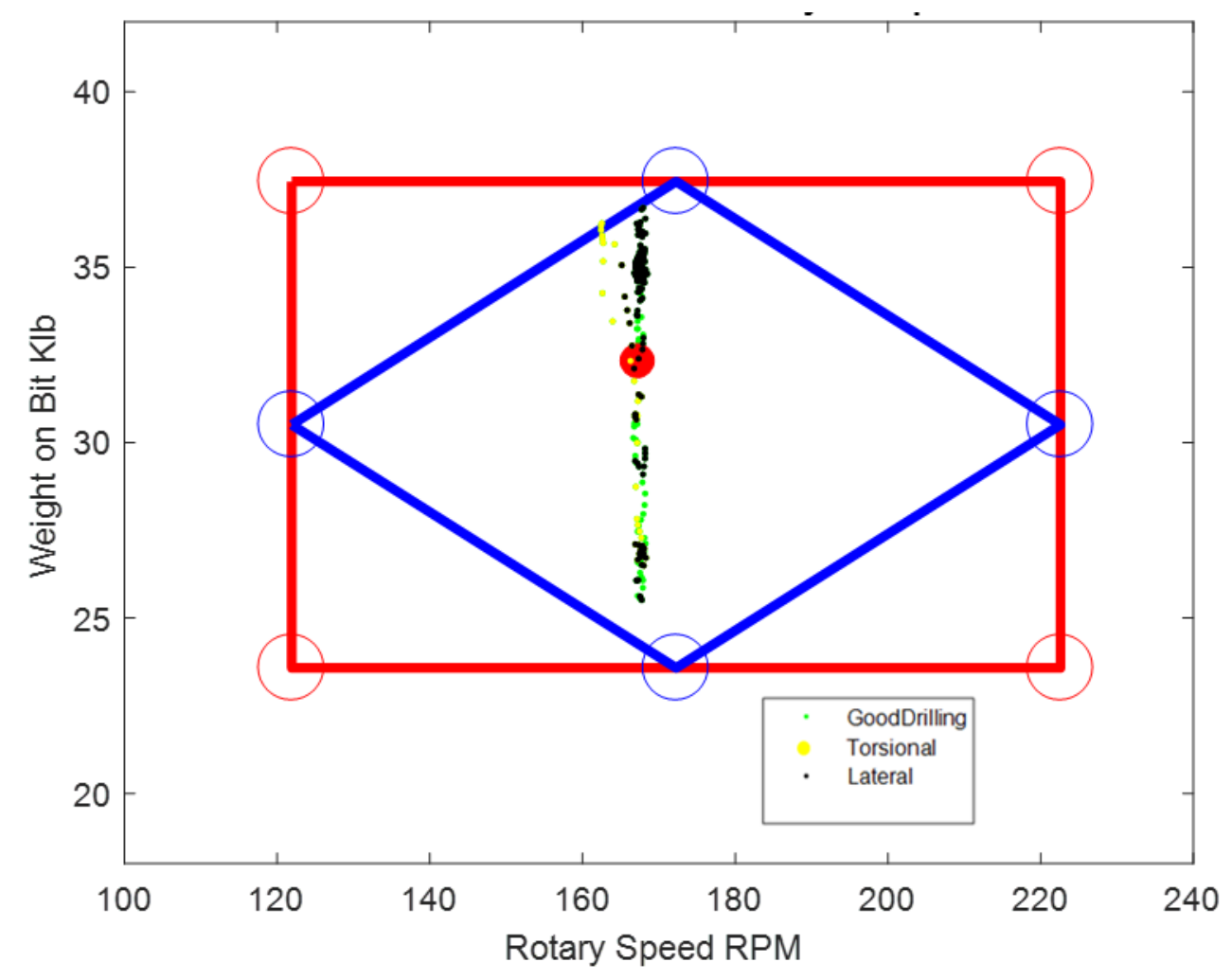

Figure 41 Classification with PCA and Safety for Update 5 
Figure 42 and Figure 43 are for update 6. The data points are also all in the optimum zone even though the downhole recordings indicate that there is good drilling, torsional and lateral vibrations.

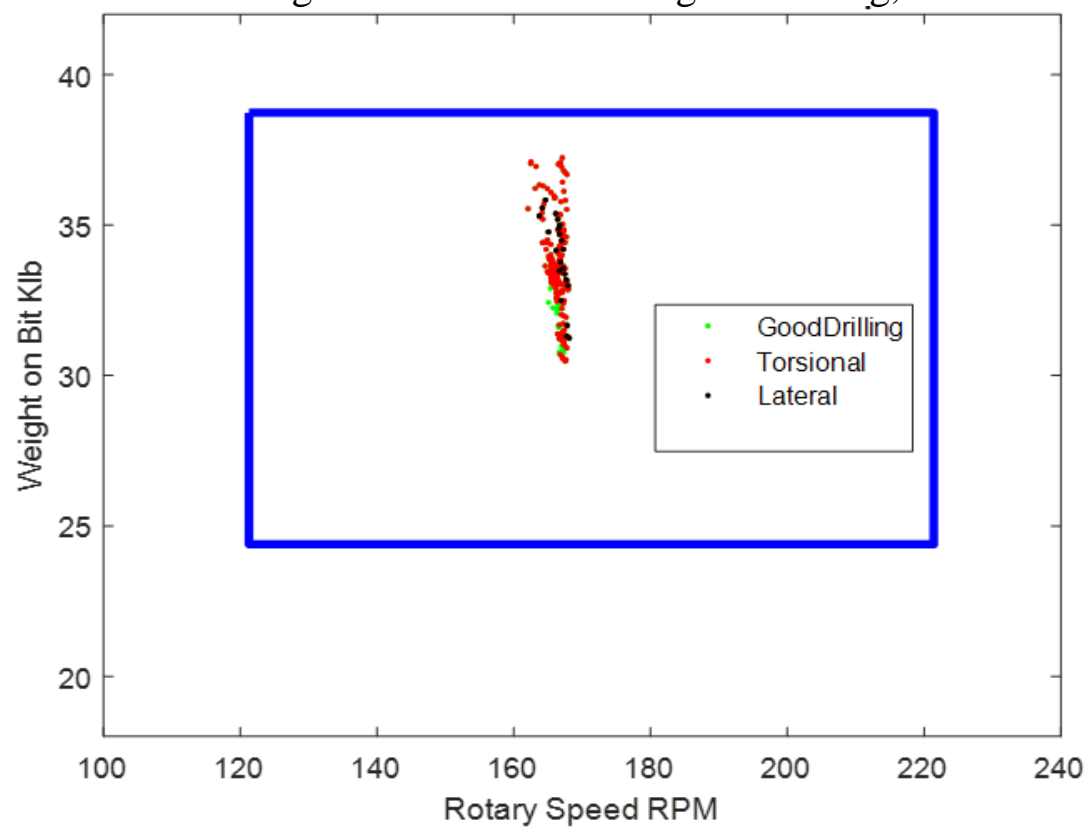

Figure 42 Classification Based on Downhole Data for Update 6

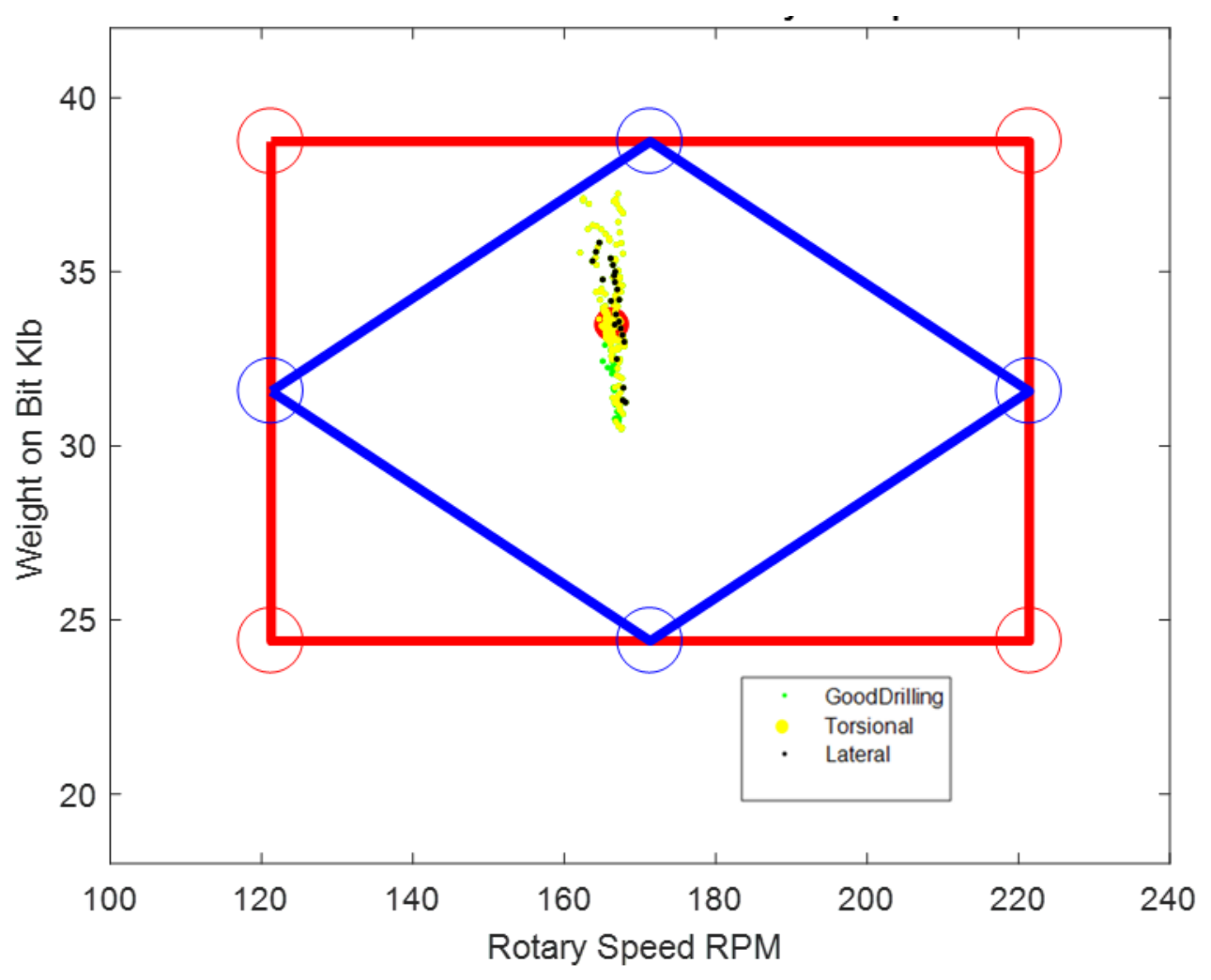

Figure 43 Classification with PCA and Safety for Update 6

Figure 44 and Figure 45 are the results for update 7. All the data points are in the optimum zone. 


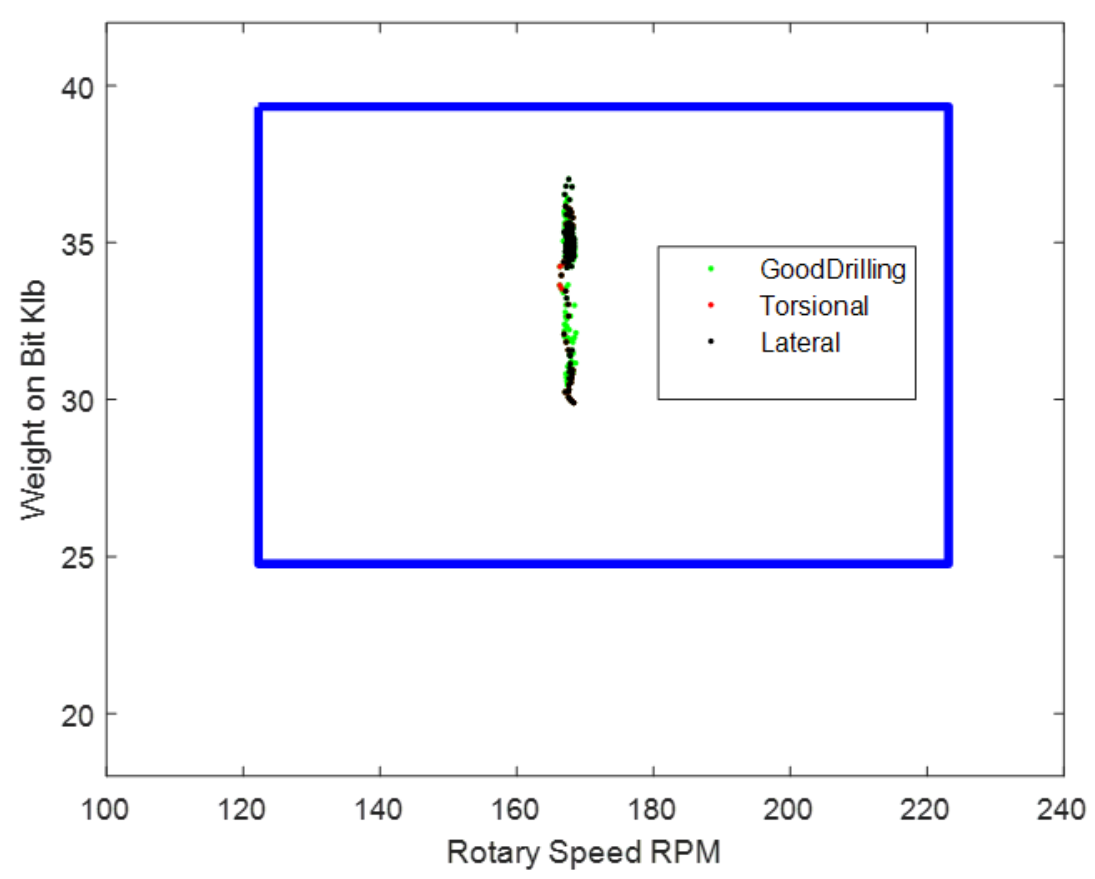

Figure 44 Classification Based on Downhole Data for Update 7

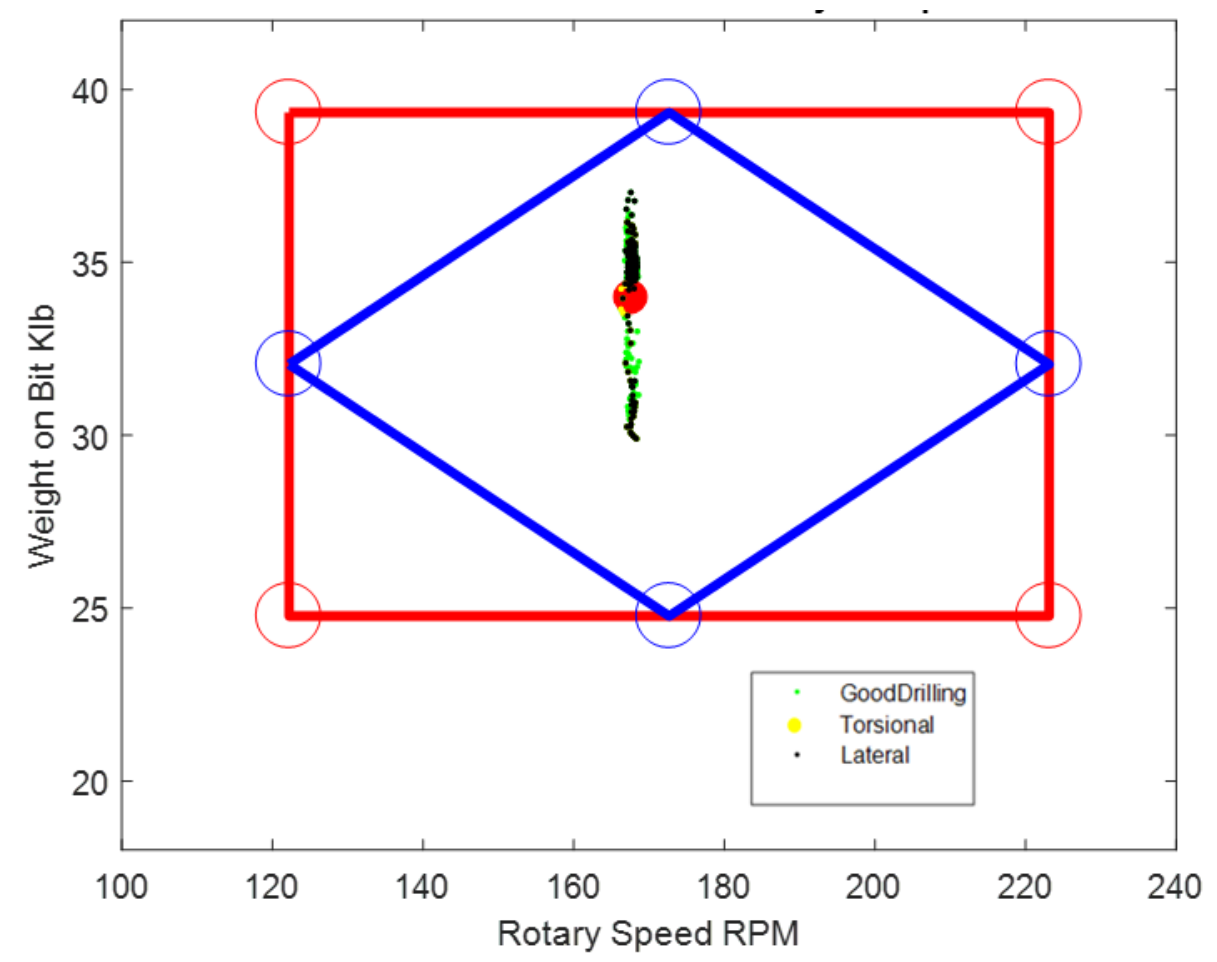

Figure 45 Classification with PCA and Safety for Update 7

One thing that is right so far is that the data points fall within the safe zone but the good drilling data points is mixed with stick slip and whirling. 
This same analysis was done for a different stand. The first update gave readings of a completely good drilling. However, the optimum zone as shown on the figure below cut out some of the data points which were potential vibration zones.

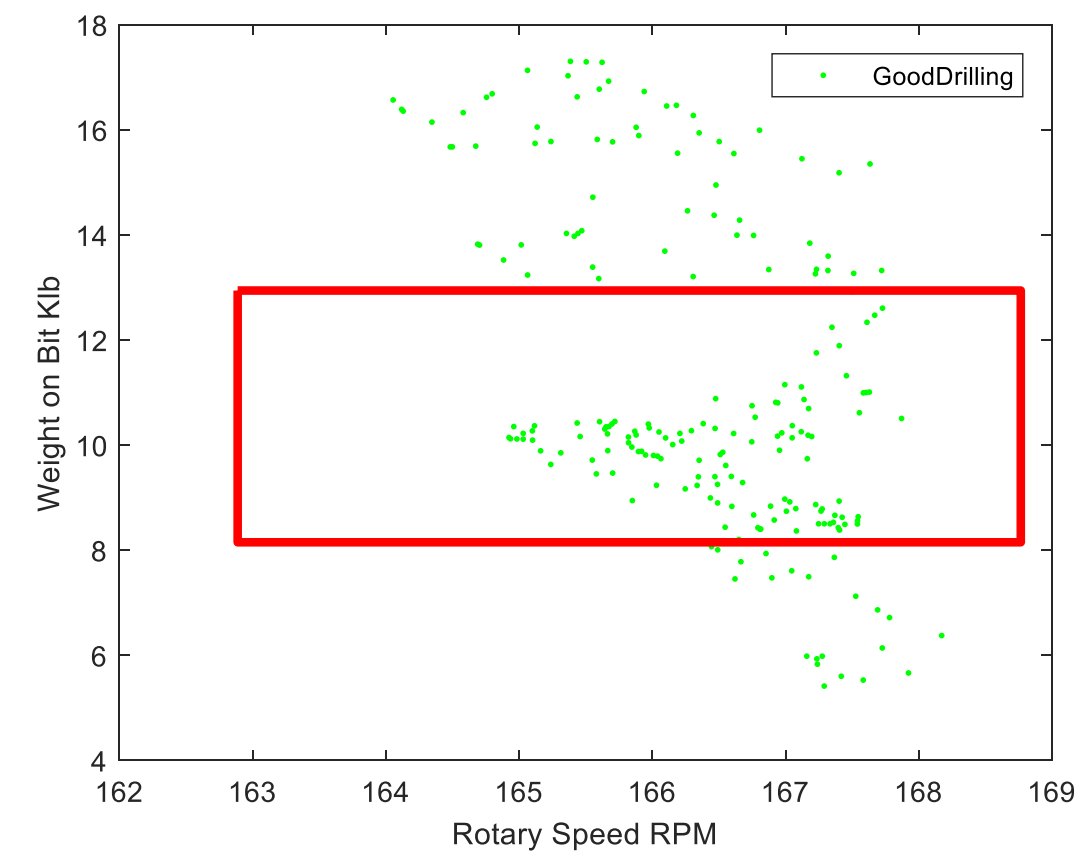

Figure 46 Classification Based on Downhole Data for Good Drilling Stand

When the safe zone analysis was done, a fewer values were safe to drill with as shown in the Figure below.

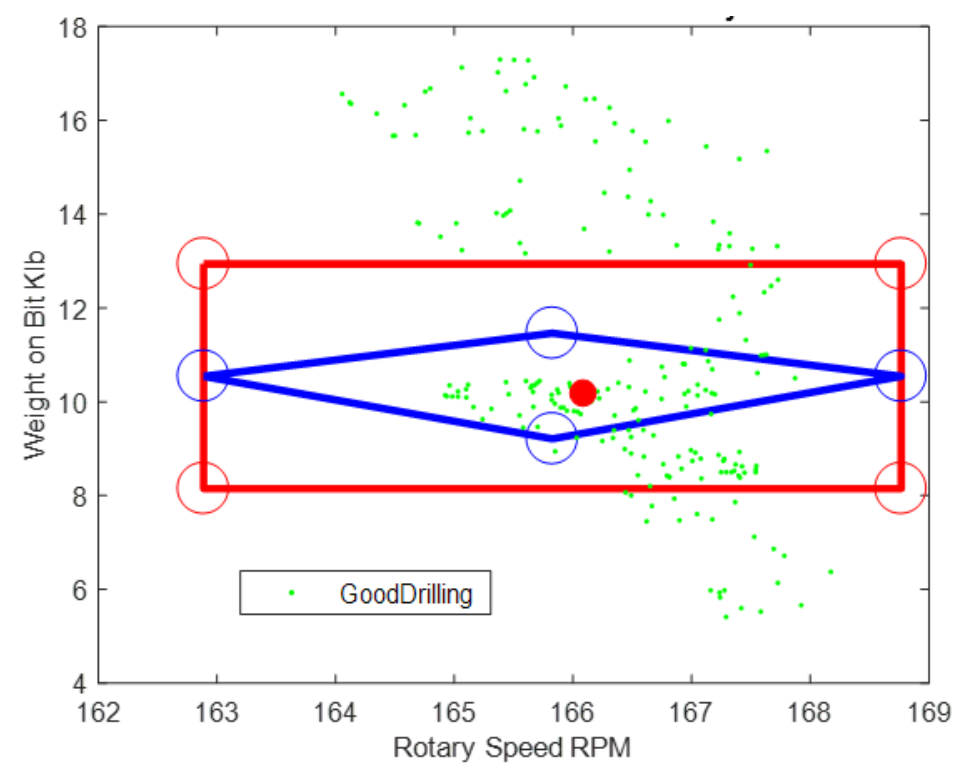

Figure 47 Classification with PCA and Safety for Good Drilling Stand 
The truth is based on the data set, the safe zone and optimum zone keeps changing. Sometimes the distinctions of different zones are clear while at other times, the distinctions are not.

\subsection{Chapter Five Conclusion}

Principal components that make up $98 \%$ of data significance were chosen to represent the original data. Updates on the safe zone and optimum zone were generated every 3.4 minutes. The optimum zone model was trained based on downhole data and tested on surface data. The clear distinction of good drilling was not seen in all cases. 


\section{Chapter Six: BUSINESS OPPORTUNITIES FOR OPTIMUM ZONE RESEARCH}

To evaluate potential business opportunities, a series of market analysis studies were conducted. In the summer of 2017, 59 customer discovery and customer validation interviews were conducted. These were based on training at Innovate Calgary under the platform of Summer Incubator Program organized by Hunter Centre for Entrepreneurship and Innovation, and Faculty of Graduate Studies, University of Calgary. This chapter will review the results of the interviews and highlight the market opportunities of starting this project as a business under the name Optimum Zone Identifier (OZI).

\subsection{Basics of Customer Discovery and Customer Validation}

The concept of customer development was first discussed by Steve Blank in the mid-90s. This method advises startups and entrepreneurs to build their products based on an understanding of what the customer wants to avoid market risks [66]. There are four categories in the customer

development concept: Customer Discovery, Customer Validation, Customer Creation and Company Building. This research has explored customer discovery and customer validation. In customer discovery, the founders are forced to "go out of the building" by going to talk with customers and finding out if the initial assumptions of what the customer wants to solve are true or not. Customer discovery can be divided into four phases as shown in Figure 48. Founders can use their findings from customer validation as a suitable sales model which can be improved upon. Customer validation can also be divided into a cycle of four phases as shown in Figure 49[66]. 


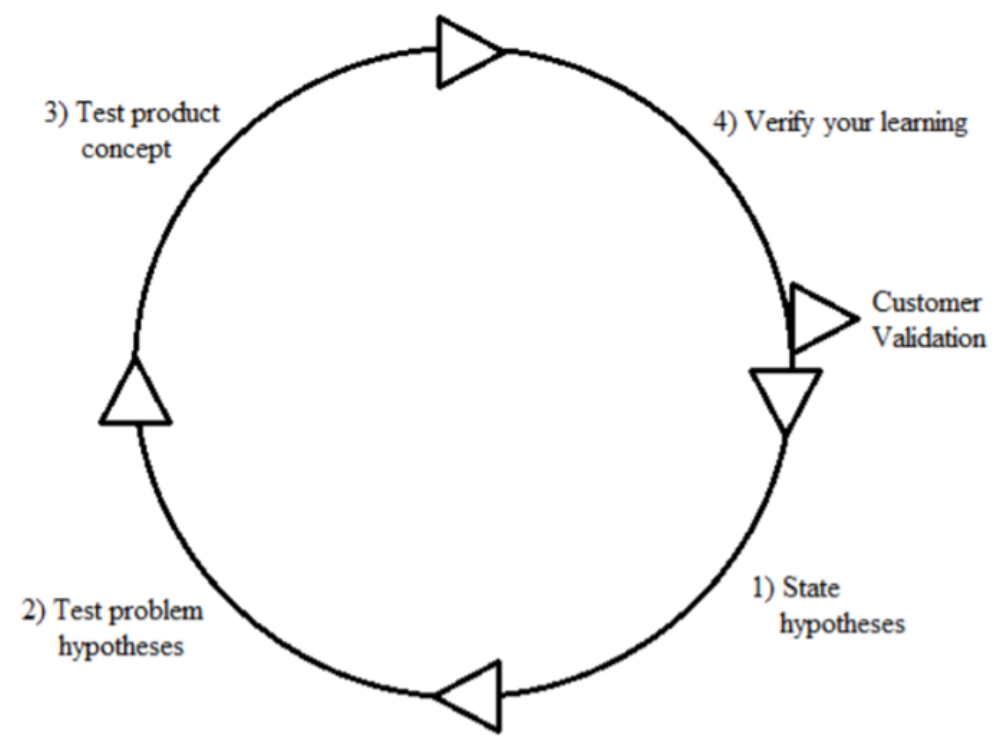

Figure 48 Phases of Customer Discovery [66]

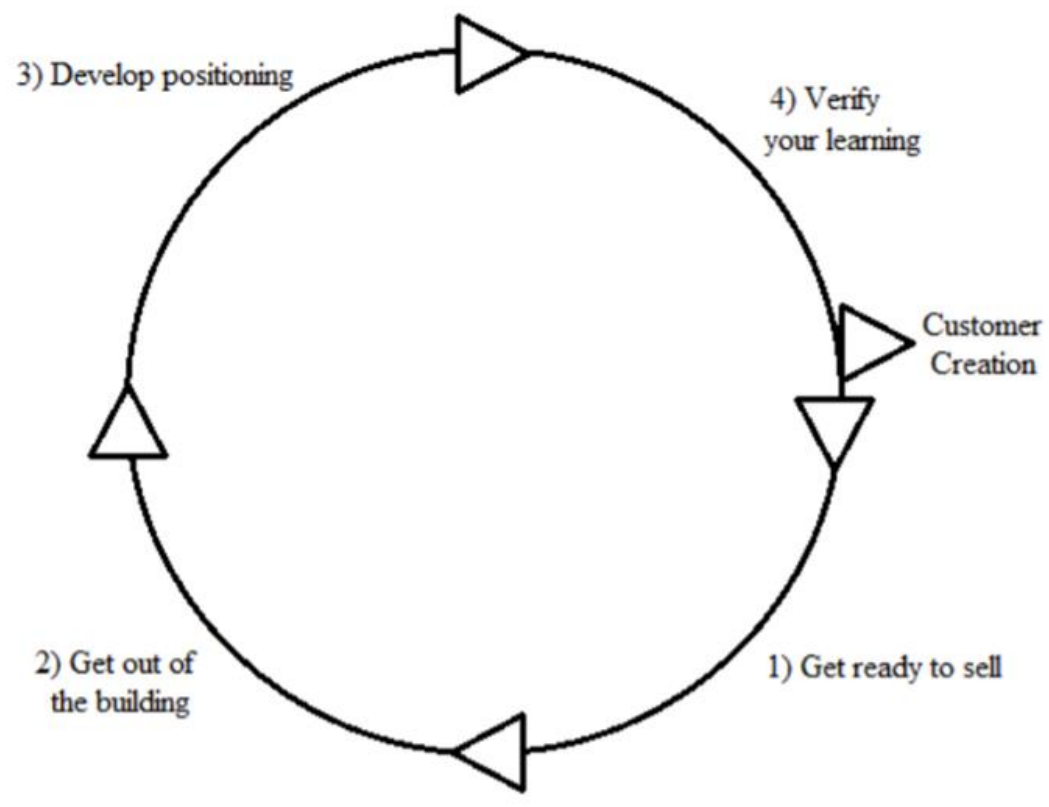

Figure 49 Phases of Customer Validation [66]

\subsection{Understanding the Needs of Potential Customers}

The initial opinion was that drilling companies need the optimum zone identifier product. There was a paradigm shift when participants at the Summer Incubator program were informed of the need to interview customers. After a couple of weeks, the interviews revealed how broad the oil industry is; there are drilling companies and there are oil producers and association for companies 
in different aspects of the oil industry. Each set of companies have different needs or view the same need from a different perspective. The major difficulty faced was to break down the oil and gas industry into components and understand how each component interacts with OZI product. The basic instruction given was that answers given by interviewee should not be compromised by telling them about OZI product. This meant all interviews conducted were based on the unique need of the interviewee. This ensured that the integrity of the comments made by the interviewee were not biased to please nor disappoint the interviewer. Successful interviews were the ones where more listening was done by the interviewer until the potential customers felt comfortable enough to reveal what their true pain was. Customer Discovery Results

Three major needs top the charts during the interview with leaders and workers of the oil and gas industry in Alberta. The most pressing need was that the Canadian oil industry is landlocked. The interviewees said $99 \%$ of Alberta crude oil product gets sold to the US at a discounted rate. In recent times, the biggest competitor is now the US who discovered shale gas. The reason Canada's oil is landlocked is because of the unapproved pipeline developments which are heavily opposed by environmentalists. The argument is that it is environmentally dangerous to transport these crude across the country, however, interviewees insist that Alberta/Canada environmental regulations are the toughest in the world already. Some interviewees went as far as claiming that major players in the oil business in the US are the ones funding the environmental movement in Canada. Interviewees collectively agree that if the Canadian oil industry can reach global markets, then the discounted rates would be removed since there would be a competition to buy Canadian crude. The second biggest issue on the minds of interviewees is the oil price. Since the recent drop in oil price, major projects have become uneconomical so they have been suspended or canceled. The concern here is that the cost of producing in the oil sands is more expensive than producing oil in other parts of the world. This means that the breakeven cost in Canada is quite high; a low oil price will adversely affect the oil industry in Canada more than in Venezuela, Nigeria, Russia or Saudi Arabia. 
The third biggest issue is the need to lower cost so that any decrease in oil price can become more bearable. The need for lower cost is everyone's business. Some costs are not controllable. The cost of rig or other production equipment may fairly stay constant or go down slowly. One factor that may drive down cost is technology advancement in the drilling industry. If newer techniques of drilling help reduce cost then the oil industry can become more profitable. The question, are the managers who cut the checks ready to spend on innovation? According to a top staff at top drilling company, one of the interviewees, the drilling industry in Canada needs to move into a digital future. It is this issue that spurs the need for products like the Optimum Zone Identifier in the oil industry.

The biggest discovery came during my $48^{\text {th }}$ interview with a general manager from Secure Energy. The initial assumption was that only oil producers conduct drilling. In this interview, it was discovered that oilfield waste management companies like Secure Energy also drill to dispose oilfield waste. They drill two to three vertical wells every year. According to the interviewee, 3 companies control about $70 \%$ of the market share. This interview triggered an increase of the customer segment to 2 . Further questioning revealed that these oilfield waste management companies subcontract their drilling activities to drilling project management companies who manage the entire drilling project for oilfield waste management companies. For instance, Secure Energy's drilling is being handled by CBW Engineering who employs Jomax to drill wells for Secure Energy. It is CBW Engineering who will decide if there is a need to acquire new software to optimize drilling. In this case, CBW Engineering would be a channel to reach Secure Energy for business.

These customer discovery results indicate that there is a possible need for Optimum Zone Identifier (OZI) since the interviewers indicate that reducing the cost of drilling will help the oil industry in Canada stay profitable even in recession times. However, there is still a need to know if OZI is one of the products they need. That is where customer validation comes into play. 


\subsubsection{Customer Validation Results}

It was validated that the operator of the drilling is the one who makes the final decision on what parameter changes can be made during drilling. In the case of the oil producing companies, the company man (representative) makes this decision while for the oilfield waste management companies, the drilling project management companies makes this decision. This information is key because the advisory that OZI generates is drilling parameter changes before or during vibration problems. Other facts validated are a pricing model and a simple business model.

After a couple of interviews, the word "license" became key to the pricing model. Most software programs are priced on a license basis. It could be either a monthly license, yearly license or per use license. Figure 50 below shows the license model presented to Innovate Calgary at the end of the summer of 2017.

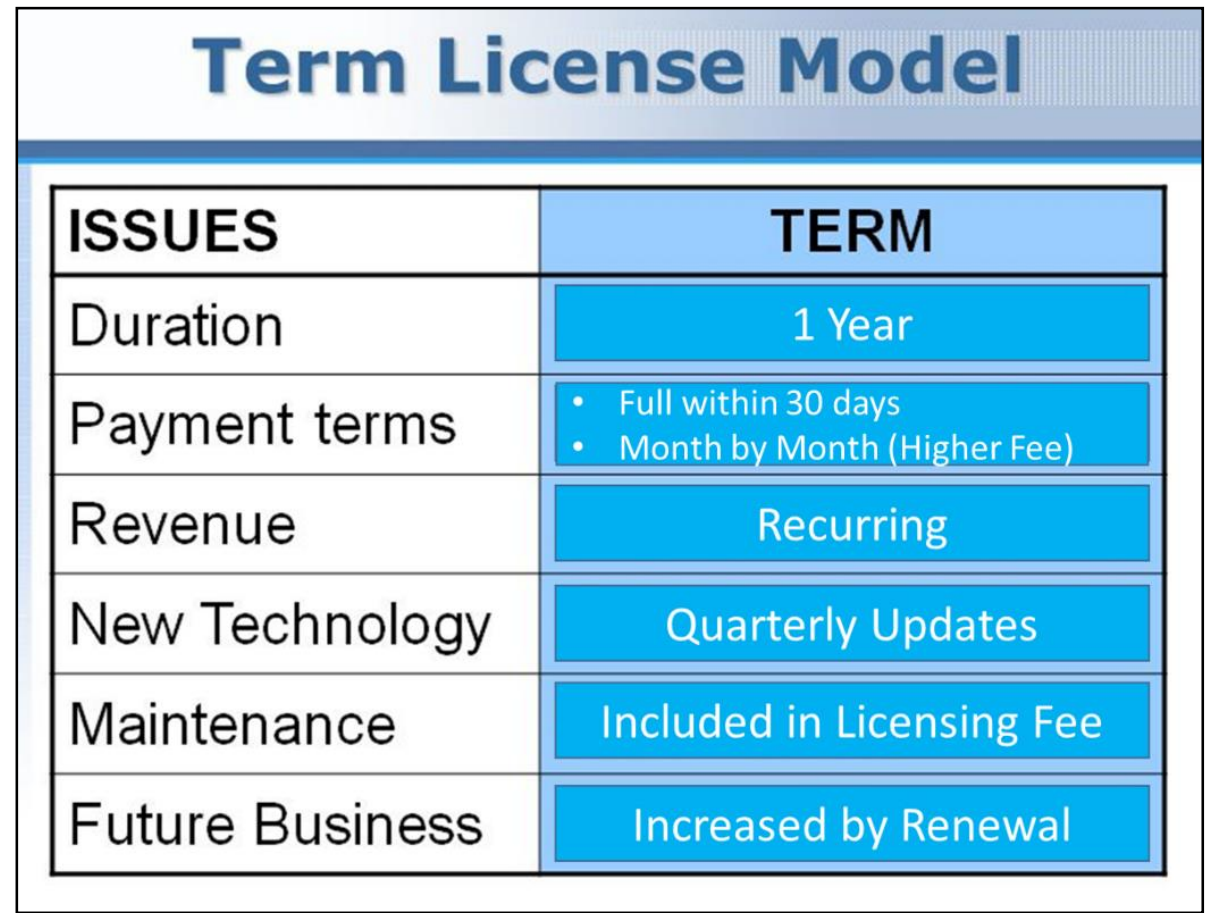

Figure 50 Pricing Model Birth from Customer Validation Interviews Summer 2017

This pricing model is value based and developed based on customer validation interviews. The concept is that if the price would be fixed; it would be derived based on the generic amount of financial savings the operator may accrue while using the software. One interviewee said he 
charges yearly payment for use of his software. His rationale was that monthly or per use basis would make him work too hard on each deal. Using a licensing manager, he can turn on or put off his company's software product when the licensing agreement is running or expired respectively. The business model derived from the interviews is quite basic. This was described by a top staff at NRGX Technologies Limited. NRGX simply uses business model that has no overhead. The figure below shows the structure of NRGX Technologies which was recommended during the customer discovery interviews. The concept is that every staff works for themselves and probably on a part time basis until the software companies becomes buoyant enough to pay salaries on a full-time basis. However, in the case of NRGX Technologies, the intention is to keep the company this way even when the company scales up. The president of this company already has twenty years oil industry work experience so this model might not be ideal for young entrepreneurs with no industry experience. Figure 52 shows the simple company structure that depicts the unique business model for NRGX Technologies.

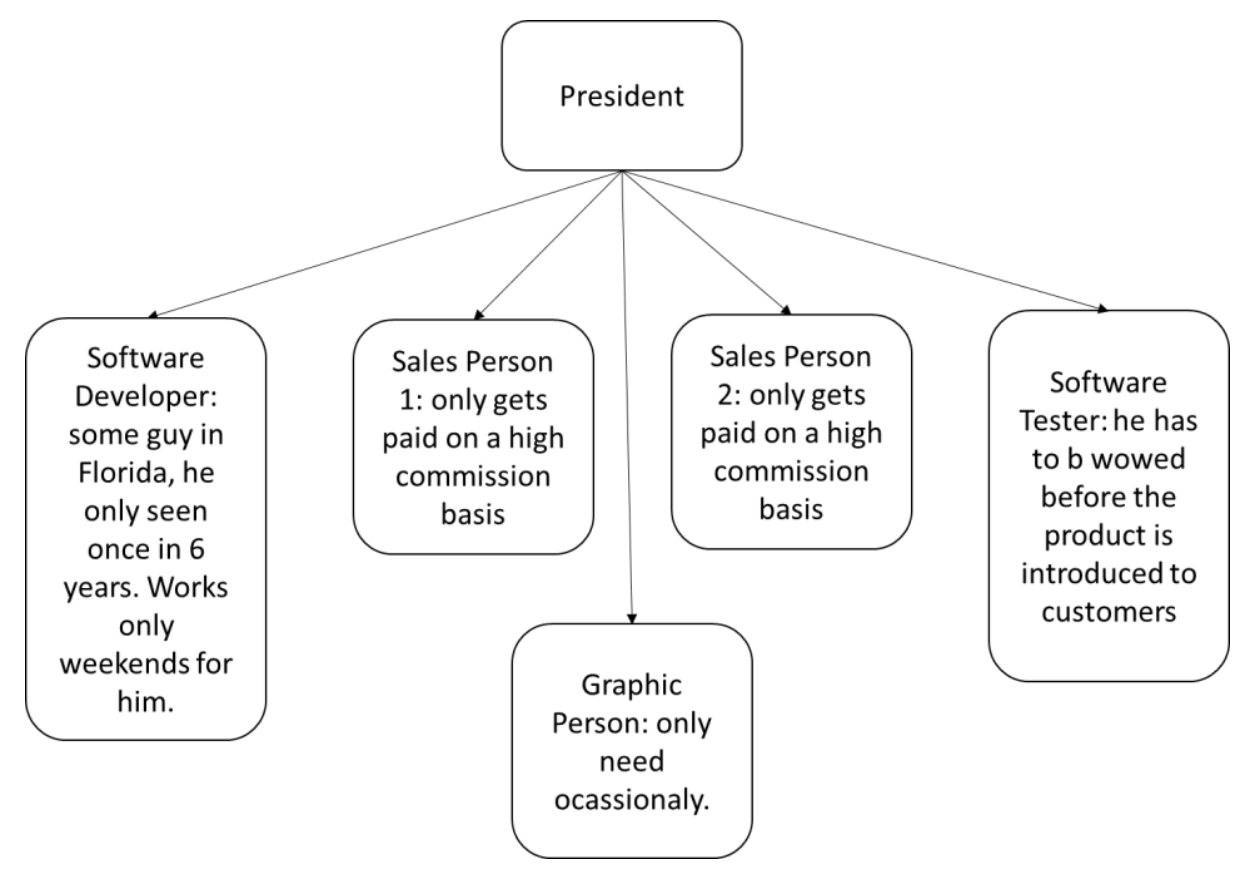

Figure 51 NRGX Technologies Company Structure based Customer Validation Interview 


\subsubsection{Adoption Life Cycle}

To begin to understand what has and could happen to software companies when they approach market in oil and gas industry, there must be an appreciation for the Technology Adoption Life Cycle (TALC). TALC is a model used by a lot of business leaders to foresee the rate of acceptance of their technology by the different customer segments based on the personality of each customer segment [67]. TALC curves like a normal distribution curve where the adoption is shown as the quantity of consumers over time [67]. As with any normal distribution, the segments (stages) are defined by a standard deviation [68].

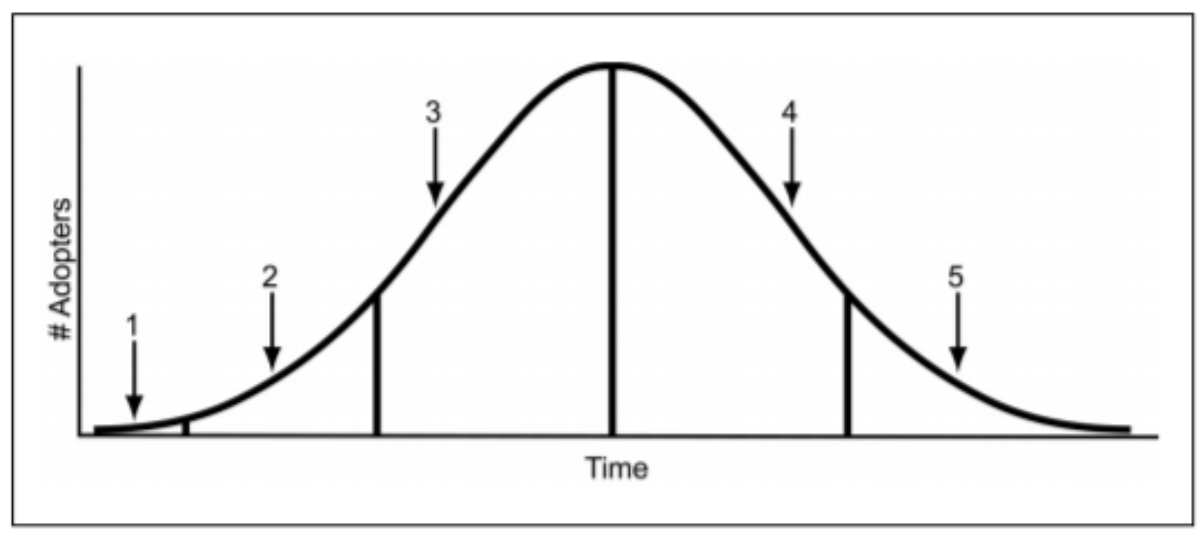

Figure 52 A Typical Adoption Life Cycle for New Technologies [68]

The personalities of the consumers in each stage are:

1. Technology enthusiasts (a.k.a. innovators): These are individuals who are interested in getting their hands on new technology and are willing to make the move to test or purchase this new technology [67].

2. Early adopters (a.k.a. visionaries): This segment of consumers are visionary minded people who are willing to commit resources to ensure the further development of an incomplete technology. They foresee the potential impact of a new technology [67].

3. Early majority (a.k.a. pragmatists): These set of people are fact based; they would not show relevant interest unless there are proven facts that the technology will deliver as expected at a competitive level. They usually go for products with a large endorsement 
from the market and industry [67].

4. Late majority (a.k.a. conservatives): These individuals are also in need of facts of the reliability of the new technology and a track record of its deliverability. They usually recognize the need for the new technology when everyone else has started using it [67].

5. Skeptics (a.k.a. laggards): These set of individuals are prone to criticizing a new technology and they prefer to stick to the old way of doing things [67].

\subsubsection{Adoption Life Cycle for OZI}

The figure shows the adoption life cycle for OZI. This is based on customer validation interviews in Summer 2017 plus other interviews conducted in Fall 2017 and early 2018.

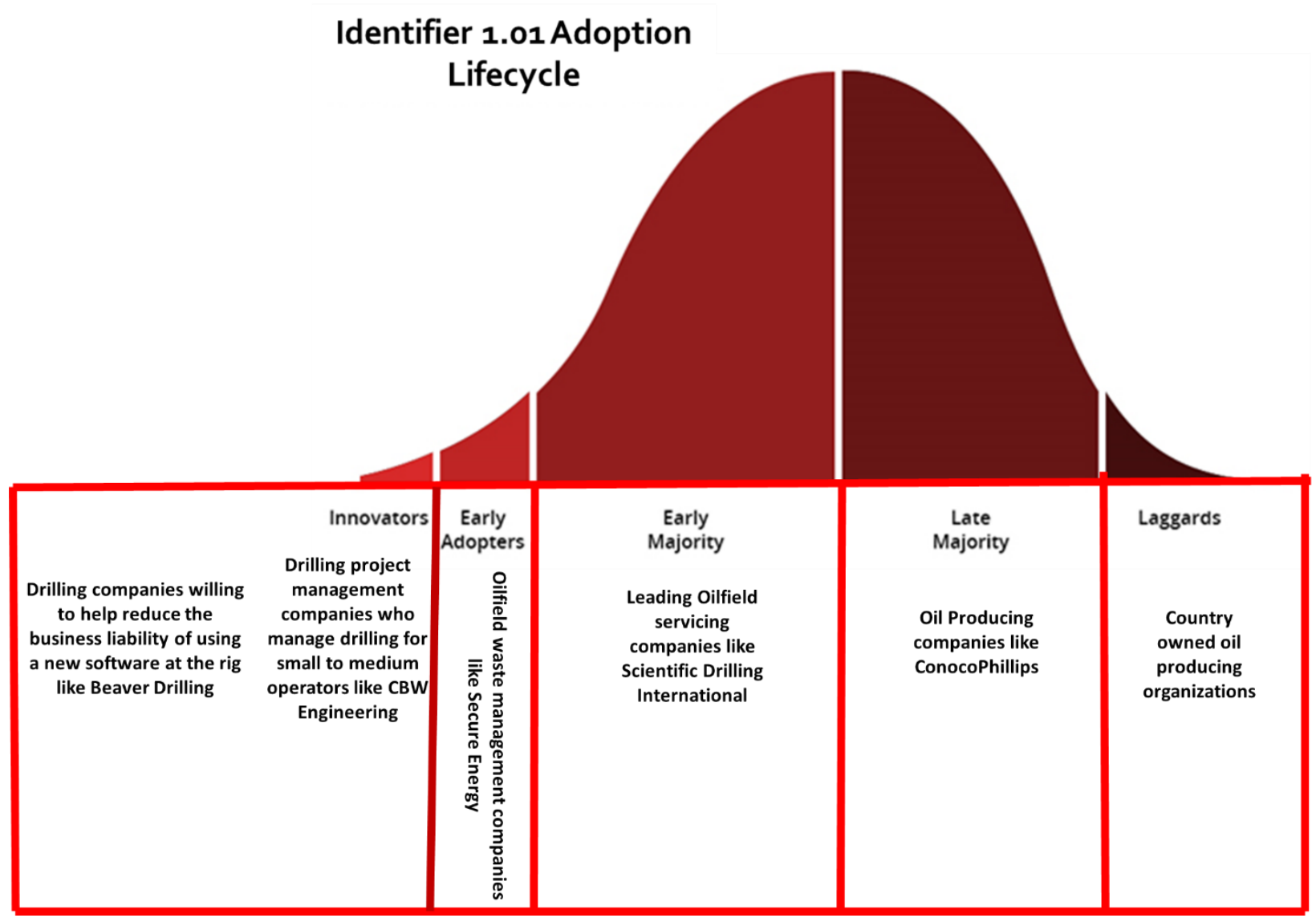

Figure 53 OZI Adoption Life Cycle

Figure 53 simply just put in one chart what customer discovery and customer validation reveals.

To approach the market, OZI as a software company needs to talk to drilling contractors for a 
potential field test then present these results to drilling project management companies who will then justify the need for the software to their clients like Secure Energy. A successful field test will also bring an oil producing company on board when the field test is favorable and widely accepted for proven methodology.

\subsection{Understanding the Market}

OZI as a company falls under the "Oil and Gas Field Services in Canada." As of November 2017, IBISWorld projects that over the next 5 years, this industry revenue will grow an annualized $2.1 \%$ to $\$ 31.0$ billion [69]. There are 5 products and services segmentation which make up $\$ 28.0$ billion revenue: Mineral drilling, exploration and deposit appraisal (7.9\%); Oil and gas exploration drilling (9.2\%); Predrilling research (15.6\%); Gas development drilling (26.1\%); and Oil development drilling (41.2\%) [69]. OZI is useful in the oil drilling development segment. The major market segmentation includes Coal and mineral extractors (9.4\%), Natural gas extractors (35.2\%), and Oil extractors (55.4\%). After 67 customer discovery and customer validation interviews; 3 customer segments exist for OZI: oil extractors. It was also discovered that oilfield waste management companies and drilling companies (rig owners) are potential customers and partners.

Startup Rationale: As demand for oil and gas increases, oil and gas extractors seek services from contractors. Over the next five years, the Oil and Gas Field Services industry in Canada is anticipated to experience slow but steady growth as natural gas and oil prices stabilize. According to the National Energy Board (NEB), domestic crude oil production will increase an annualized 3.0\% between 2017 and 2022, and as a result, the Oil Drilling and Gas Extraction industry in Canada is forecast to grow at an annualized $1.3 \%$ over the next five years [69].

Market Opportunity: The drilling and support services subsector, like the upstream oilfield services industry in general, is subject to the cyclicality, volatility and seasonality that characterize the Exploration \& Production, E\&P, industry [70]. This subsector benefits from the fact that E\&P companies must continually drill to and new reserves and replace and increase production from 
existing reserves to maintain viability [70].

The Market Gap: According to IBISWorld, in the 10 years to 2022, the number of enterprises in the Oil and Gas Field Services industry in Canada is projected to steadily increase at an annualized rate of $4.4 \%$. While the number of enterprises is anticipated to rise, many new entrants are small operators with minimal market share [69].

\subsubsection{Customer Value Proposition Canvas}

The value proposition canvas is a structured way of designing the value proposition of the product to fit into each customer segment. For every pain, the value proposition canvas gives an opportunity to state what the pain relievers are [71]]. For the gains expected by the customer, there are corresponding gain created by the product. The customer value proposition canvas feeds into the business model canvas.

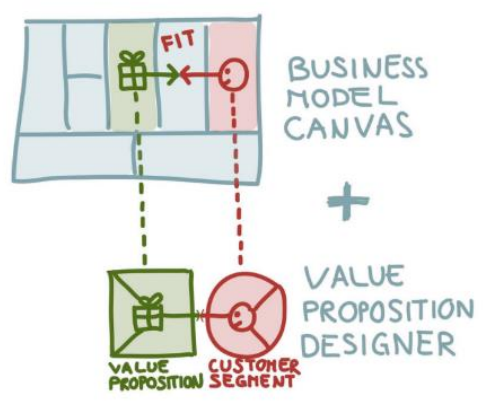

\section{Figure 54 Equating Business Model Canvas with Value Proposition Canvas [71]}

The goal of the Value Proposition Canvas is what the start-up scene calls product-market fit or problem-solution fit. The figure below shows the standard customer value proposition canvas. 


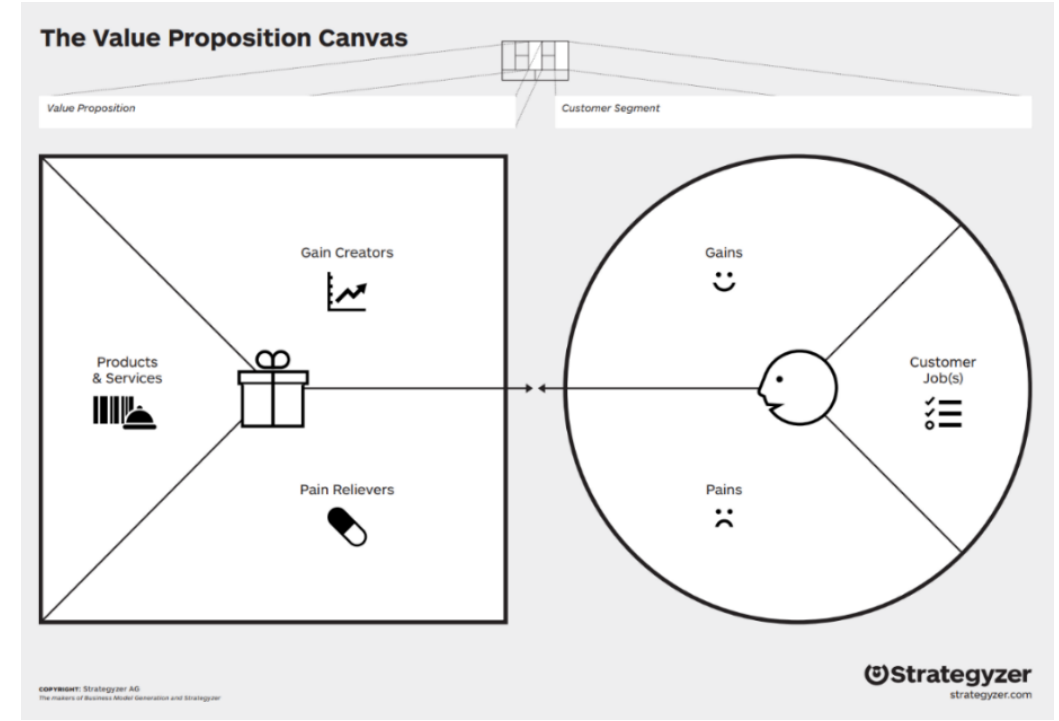

Figure 55 The Value Proposition Canvas (VPC) [72]

Customer Jobs: This refers to the task the customer is trying to get done or the problem the customer is trying to get solved. This is the duty they are doing that they want to ease by purchasing thee product the entrepreneur is proposing.

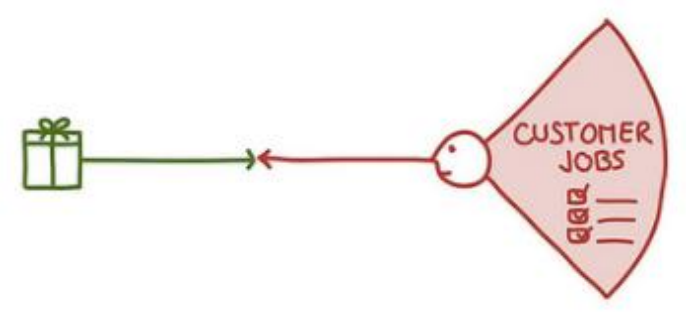

Figure 56 Customer Jobs Part of VPC [71]

Customer Pains: This refers to the pain or negative satisfaction that comes when the customer is involved with the specific task identified in "customer jobs." 


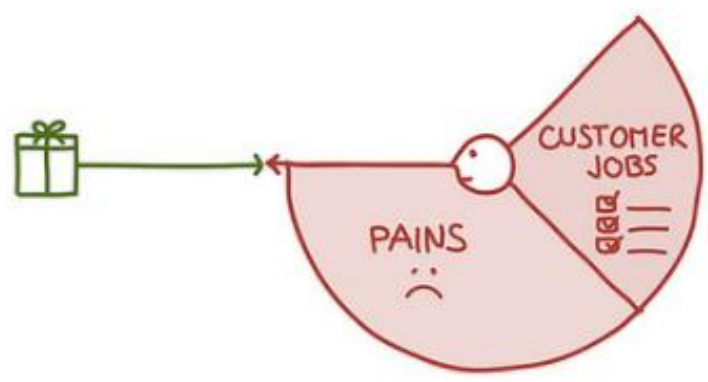

Figure 57 Customer Pain Part of VPC [71]

Customer Gains: These are the cost savings, emotional excitement or benefits the customer expects to gain from doing the task more efficiently.

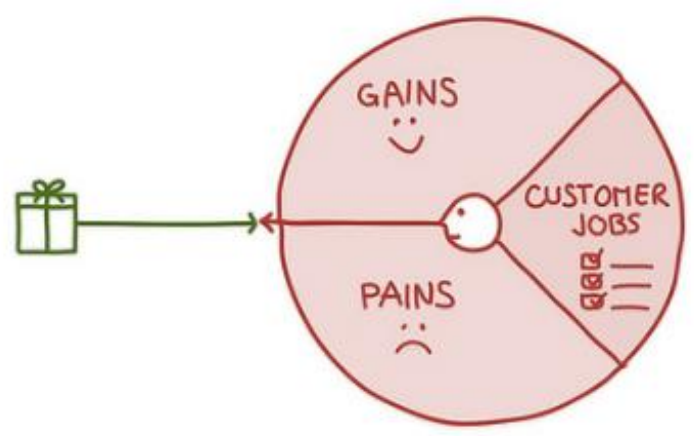

Figure 58 Customer Gain Part of VPC [71]

Products \& Services: This refers to the value proposition that achieves the same results the customer is trying to attain by doing the task in customer jobs.

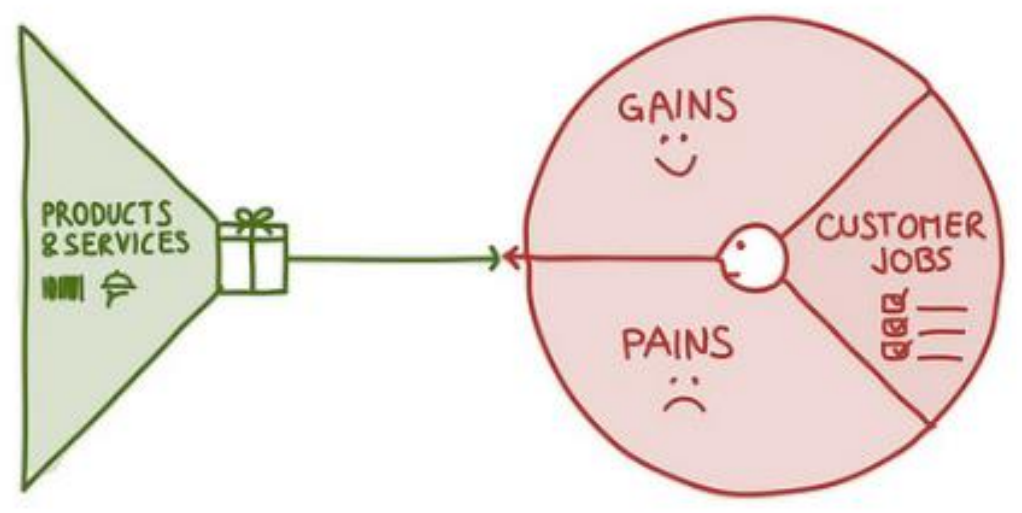

Figure 59 Products and Services Part of VPC [71] 
Pain Relievers: The value proposition is now targeted towards alleviating the pains the customer endures when carrying out the task. In this case, the product is aimed at reducing the pain of doing the customer's task. This pain would have been identified by the customer during a customer discovery interview.

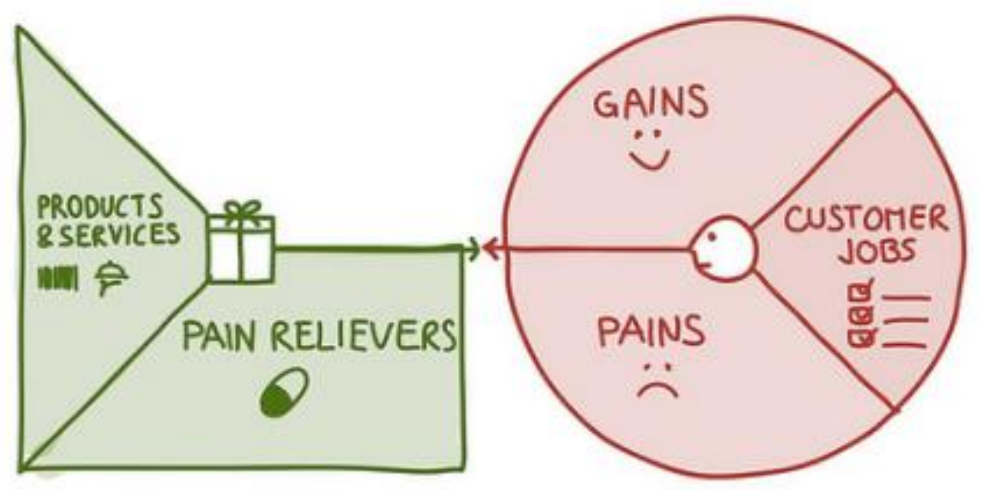

Figure 60 Pain Relivers Part of VPC [71]

Gain Creators: This refers to how the value proposition can be positioned so as to achieve a more efficient and less expensive result from the task the customer is embarking on. In the case the product fits into the ambitious mind of the customer.

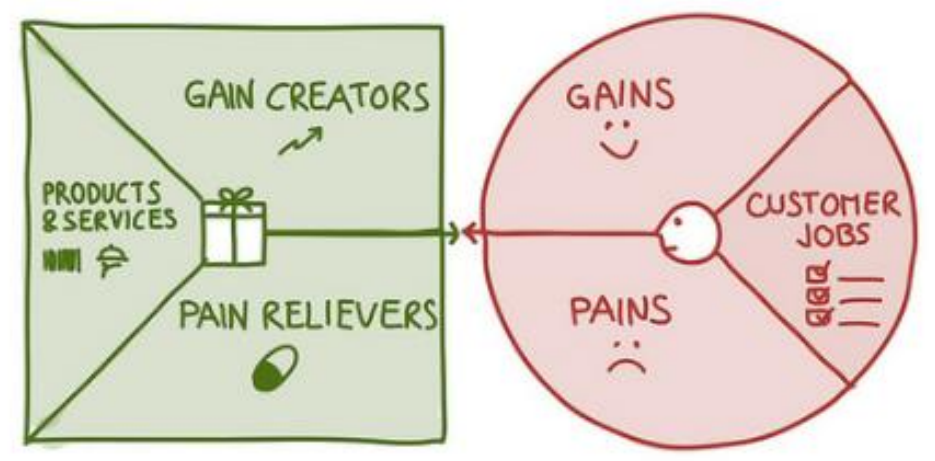

Figure 61 Gain Creators Part of VPC [71]

\subsubsection{Value Proposition for OZI}

The average cost of drilling a well is $\$ 100,000$ per day. NPT accounts for 20 percent of rig time which means $\$ 20,000$ per day [84]. It is already known that vibrations account for an average of 20 percent of NPT which means $\$ 4,000$ per day [84]. It is safe to assume that the value saved by OZI is $\$ 4,000$ per day. On a value based pricing model, the price of OZI would be an agreed 
fraction of $\$ 4,000$. For OZI, 3 customer segments have been identified: oil extractors, oilfield waste management companies who conduct drilling, and drilling companies who drill for oil extractors. They 3 main needs on the minds of these customers is 1.) How to reduce operation time, 2.) How to optimize through accurate real-time decision making, and 3.) How to understand downhole dynamics without spending too much on downhole tools. OZI can serve these needs by offering real time drilling parameters optimization, predicting unexpected vibration events, and real-time vibration measurement.

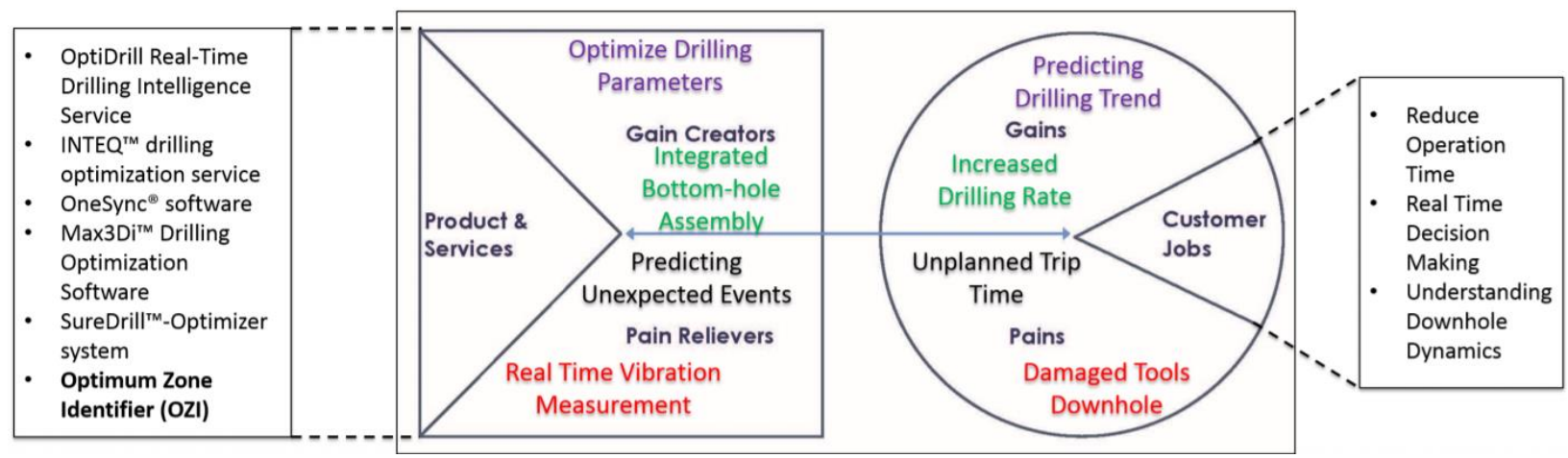

Figure 62 Customer Value Proposition Canvas for OZI

\subsection{Business Model Canvas (BMC)}

Business Model Canvas is a strategic document that documents the mind of the business. It is a great tool for lean startups and already existing firms. It is a consolidated table with nine elements describing a company's value proposition and customer segments, and how the business revolves around these two pieces leading to a revenue stream and cost structure [74]. It gets the management of a firm to start thinking of how the company can function as a complete organism. Based on his work on Business Model Ontology, Alexander Osterwalder designed the Business Model Canvas [73]. Figure 64 below shows the business model canvas for OZI and the subsequent sections will reveal the thought process for developing this BMC for OZI. 
The Business Model Canvas

Designed for. OPTIMUM ZONE IDENTFIER

Designed by: ETAUE DARLINGTON

Date: $20.04-2018$ Version: 5

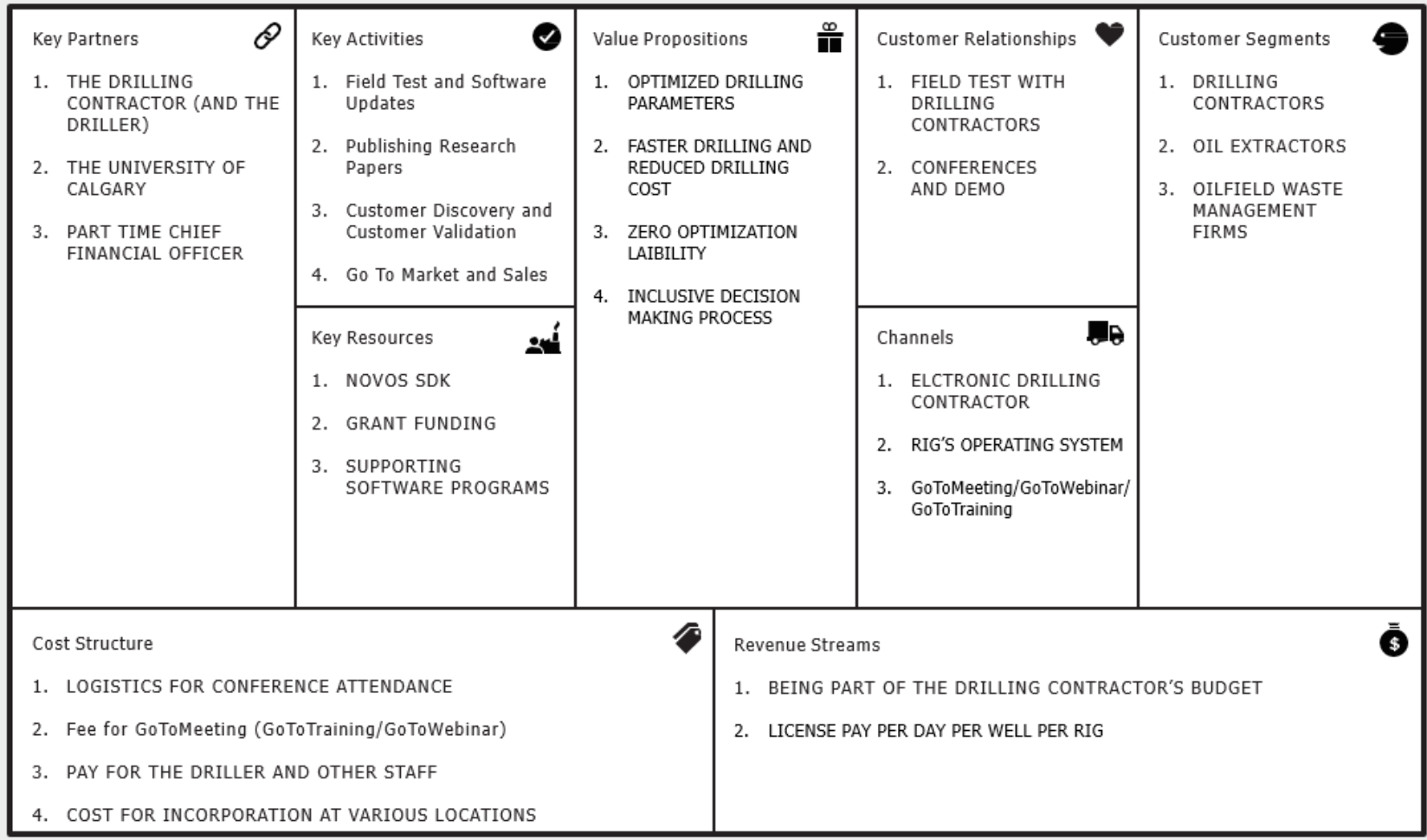

(a) (ㄷ) (2) (-) (1)

Decloreo by

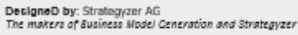

(EStrategyzer

Figure 63 Business Model Canvas for OZI

\subsubsection{Value Proposition in BMC for OZI}

\section{The Pains of the Customers}

It cost an average of $\$ 100,000$ to drill a well per day. If a severe vibration problem occurs while drilling, the drilling pipe and other tools down-hole may be damaged. The driller may have to remove the entire tools from the hole and replace them. This could take several hours or even days.

For a well as deep as 4000 feet, it may take 1 to 3 days to bring out the tools and replace them.

That would mean about $\$ 300,000$ lost. Imagine if this problem occurs in several wells.

\section{OZI Pain Relievers}

Optimum Zone Identifier is a software service that offers the driller optimized drilling parameters 
to work with before or during vibration problems.

\section{The Gains Needed by the Customers}

Reaching the target depth faster is the dream of every driller. The oil extractor aim is to ensure efficient drilling that uses a slim budget to achieve the target well inclination and azimuth without non-productive time and without invisible lost time. In summary, increasing the rate of penetration safely using the best of technologies is the pie the oil industry longs to see anytime they drill a well.

\section{The Gain OZI Creates}

Based on this research, a projection of at least 10 percent of the time it takes the driller to reach the target depth can be saved. This means the driller will get to the target depth faster and stay close to his planned path before drilling started. This would mean cost savings as well.

\subsubsection{Customer Segments in BMC for OZI}

\section{Segment 1: Drilling Contractors}

One of the earlier concerns was how OZI will get the drillers involved in its business. Obviously, the drilling contractors want to increase their profits by increasing the cost of drilling while OZI are proposing reducing the cost of drilling through its software. It turns out that the driller who works for the drilling contractor is the one who would operate OZI's software in the rig. If OZI's direct customers (the oil extractors) need feedback from the users (the drilling contractors) who is also a competitor to OZI then failure is inevitable. Therefore, making the drilling contractor a customer, makes a lot of sense. At this point OZI had to tweak the pattern. 


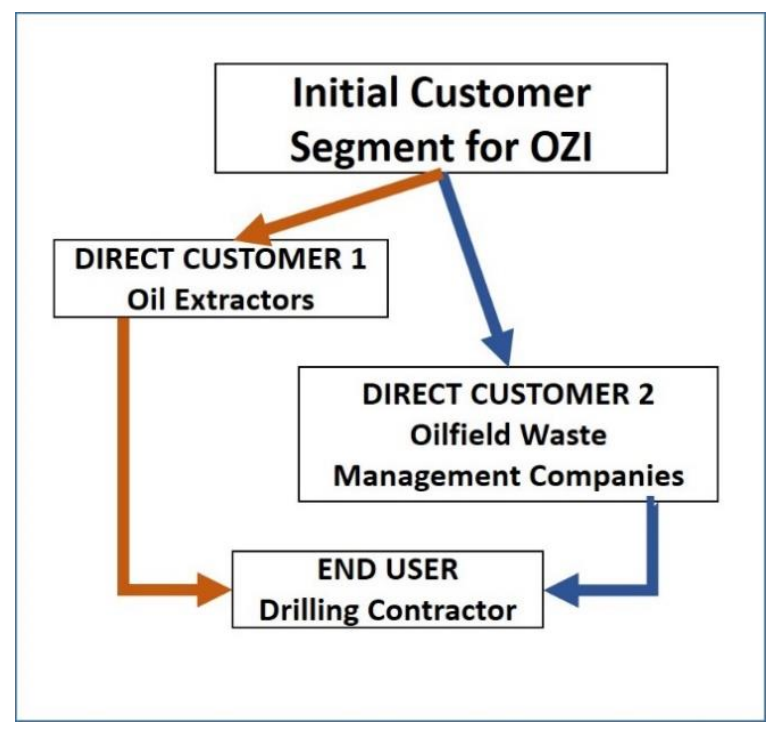

Figure 64 Initial Customer for OZI

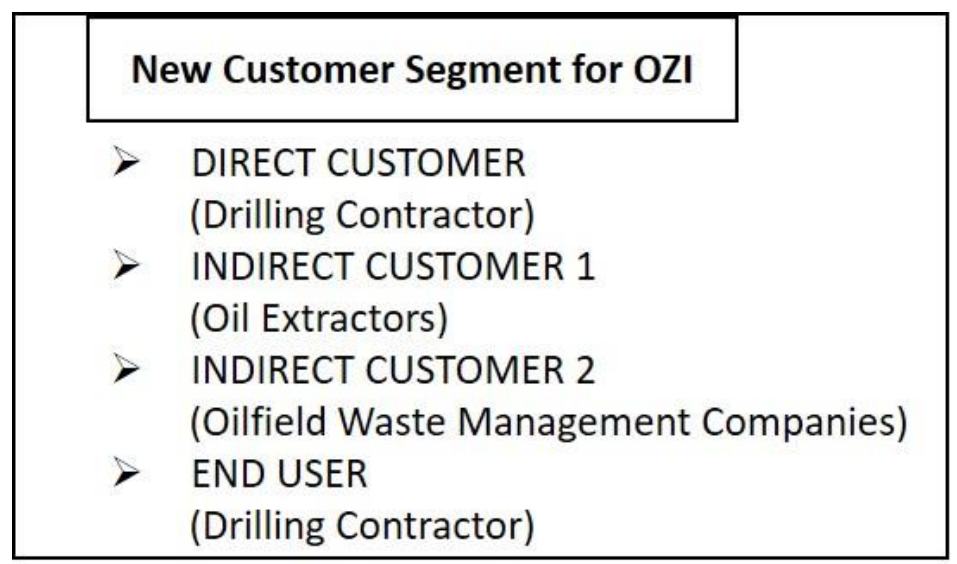

Figure 65 New Customer for OZI

The drilling contractors are now OZI's direct customers and also its end users. OZI ispartnering with a drilling contractor to run a field test in the summer of 2018.

\section{Segment 2: Oil Extractors}

The New concept has the oil extractors as OZI's indirect customers. OZI have to connect with the oil extractors after its software has been validated with the field test partners. The oil extractor to reduce the time of drilling and loss of tools down-hole. That is where OZI's software comes in as a pain reliver. 


\section{Segment 3: Oilfield Waste Management Firms}

During the 48th customer interview, we discovered that Secure Energy also drill to dispose oilfield waste. They employ drilling contractors as well.

\subsubsection{Customer Relationships in BMC for OZI}

\section{Field Test with Drilling Contractors}

OZI is currently scheduled to do a field test with an industry partner in the Summer of 2018. The partner is a drilling contractor and therefore in its first customer segment. The aim of the field test is to validate the methodology OZI's software introduces.

The relationship OZI hope to generate with other customers in this segment is to show them results of field tests or do a field test with them. However, this gives the drilling contractor an upper hand when sales are eventually made to oil extractors. The drilling contractor will have a higher portion of revenues because they will agree to bear all the consequences of any mishap when OZI's software is in use. This means zero liability comes Optimum Zone Identifier company when its software is in use.

In order to reduce the burden on the drilling contractor, OZI have made the use of its software only as an advisory system where the driller at the drilling console still makes the final decision after recommendations have been made by OZI.

\section{Conferences and Demos}

For oil extractors and oilfield waste management companies, OZI need to gain their trust so constant communication with them is necessary. If there are positive results from the field test show that the benefits by far outweigh the cost of using OZI software, then the gain can be itemized clearly. OZI will then publish papers and invite potential customers for demo sessions.

\subsubsection{Channels in BMC for OZI}

\section{Electronic Drilling Recorder (EDR)}

When used to its fullest, it is a robust system of computers, instruments, and monitoring equipment networked around a rig. OZI can draw real time surface data from the EDR. 


\section{Rig's Operating System}

This system is synonymous with what Microsoft is to a computer. In our case, we are building an app that would work with NOV's operating system. NOVOS ${ }^{\mathrm{TM}}$ is the industry's only reflexive drilling system, automating repetitive drilling activities, benefiting contractors by allowing drillers to focus on consistent process execution and safety, and benefiting operators by optimizing drilling programs.

\section{Software for Communication and Training}

OZI will use this software to constantly communicate with the rig in case of any software issues or any need for presentations. The software should be a platform for online meeting, desktop sharing, and video conferencing that enables the user to meet with other computer users, customers, clients or colleagues via the Internet in real time.

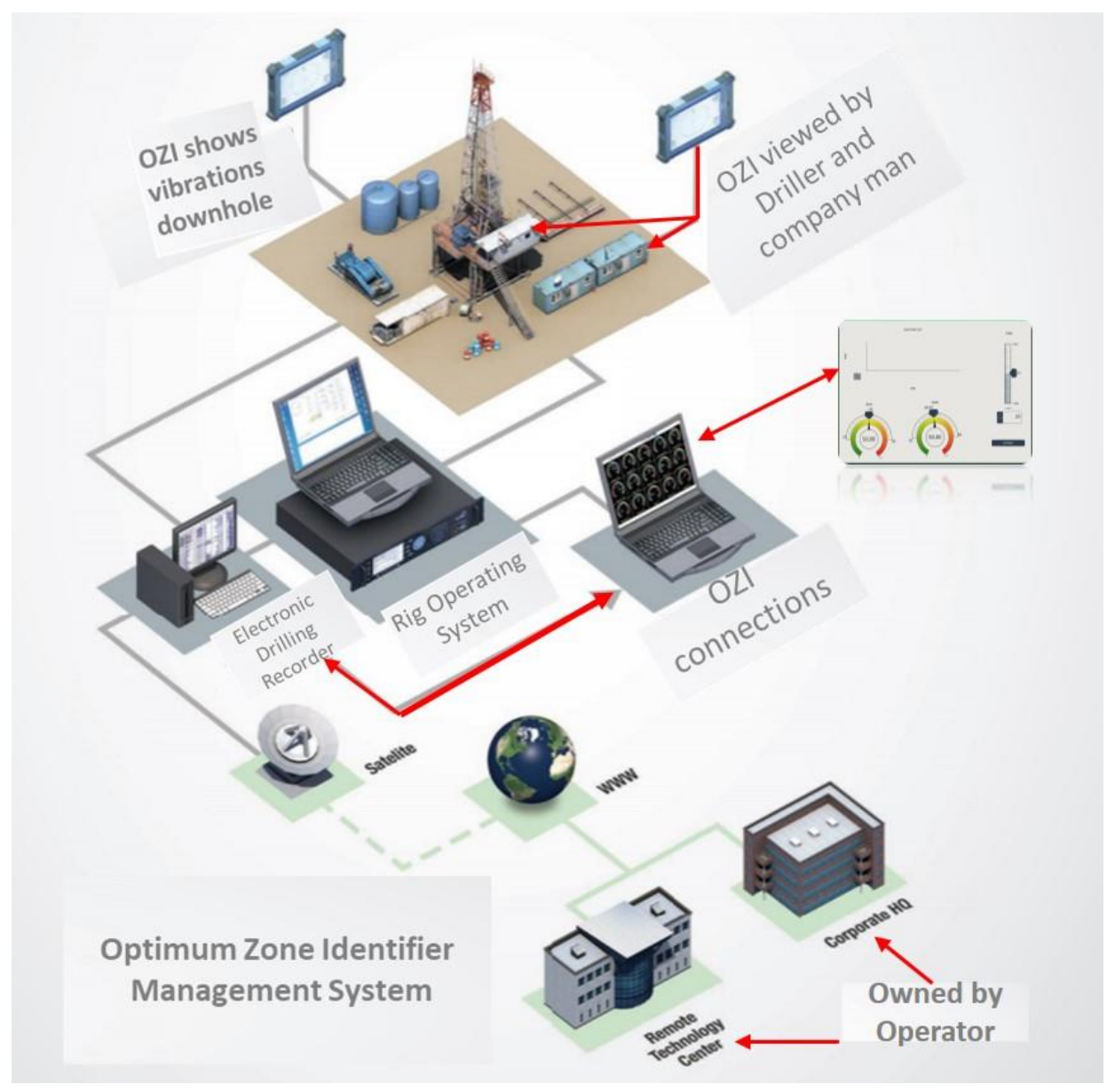

Figure 66 Management System for OZI 


\subsubsection{Key Partners in BMC for OZI}

\section{The Drilling Contractor (and the Driller)}

These companies are playing 3 roles for us. They are OZI's first point of contact as OZI work towards commercialization. The drilling contractors will be OZI's early adopters and eventually its end users. Their worker, the driller at the rig, is also a key part of OZI's business model and its payroll as well.

\section{The University of Calgary}

Staying at the university saves the intellectual property (IP) owners a lot of cost especially when legal agreements are being drawn up. These IP owners will form the core of the ownership of OZI. The IP owners will also benefit from the university's resources for research.

\section{Part Time Chief Financial Advisor}

As OZI approach going to market after the field test, OZI would need an experienced financier who can gives the directions we needed in terms of receiving investments, signing licensing agreements, employing administrative staff and securing new funding.

\subsubsection{Key Activities in BMC for OZI}

\section{Field Test and Software Updates}

OZI have a field test scheduled for the summer of 2018. This is special for OZI because the IP owners get to validate the effectiveness of OZI's software and make necessary changes to methodology based on the experience. Anytime OZI's software is being deployed a customer, OZI will also be updating its software with new learnings from the field work.

\section{Publishing Research Papers}

OZI would keep developing new research papers and expanding its publicity by attending conferences and sharing its ground-breaking discoveries.

\section{Customer Discovery and Customer Validation}

Since the summer of 2017, just over 70 customer discovery and customer validation interviews (partly for Summer Incubator program organized by Hunter Centre for Entrepreneurship and 
Innovation/Innovate Calgary/Faculty of Graduate Studies) have been conducted. More interviews are needed in order to profile OZI's customers and know how to get OZI's product in front of key decision makers.

\section{Go To Market and Sales}

OZI intend to start making sales as soon as its field test has been concluded. OZI already have a company in China showing interest and OZI will work towards making sales in Europe and Saudi Arabia.

\subsubsection{Key Resources in BMC for OZI NOVOS SDK}

The University of Calgary recently signed up to have access to use NOV's Operating System software development kit. The app that the IP owners will develop should be compatible with NOVOS SDK so that when OZI's software is in use at a NOVOS rig, the software can function since it is already built in the SDK.

\section{Grant Funding}

To employ staff as OZI expand towards an ever-growing market, there is need to receive grants which tie to OZI's research and business milestones. Key staff like legal advisor, part time chief financial officer, business development expert in drilling and a software developer are necessary.

\section{Supporting Software Programs}

OZI need the following software programs to function our business seamlessly: WITSML software, accounting software, remote access and support app, project management software, document management software, and a license manager software.

\subsubsection{Cost Structures in BMC for OZI}

\section{Logistics for Conference Attendance}

One of the best ways to get a lot of customer interviews done swiftly is to attend conferences and present technical papers. In a year, there are drilling conferences in the Middle East, Europe and North America. The IP owners attended IADC/SPE Drilling Conference and Exhibition 2017. In 
that conference, the trends in similar vibration research were discovered. In two days at the Global Petroleum Show Calgary 2017, 30 customers were interviewed.

\section{Pay for The Driller and Other Staff}

In order to maintain the integrity of the operation, the driller who works for the drilling contractor would be part of our payroll until OZI have globally proven that its software and optimization analysis is a standard and reliable. OZI also need money to pay staff especially the part time chief financial officer.

\section{Costs for Incorporation at Various Well Locations}

Depending on the rules of different countries, OZI may need to incorporate its company when deploying its software in foreign countries. OZI is expecting to expand its reach to Saudi Arabia, China and Europe. OZI will keep most conversations online but eventually OZI will need to have staff and facilities on ground. That would require additional startup costs.

\subsubsection{Revenue Stream in BMC for OZI}

\section{Revenue Stream 1: Being Part of the Drilling Contractor's Budget}

Perceptual Maps helps in identification of ways to have a unique path to approaching the market. Since most of OZI's competitors are major players in the industry, OZI can partner with the drilling contractor and be part of their total package they give to the oil extractor. The only challenge would be how the drilling contractor will justify what value OZI adds on monetary terms. The enigma is that so many variables apart from vibration can cause loss of drilling time so when drilling time is saved, how would one know which solution was the source of time gain.

However, when this model is successfully understood, the drilling contractor would be able to sell the product OZI to the driller per use and show a cost benefits analysis of what the producer has to gain by incur that extra cost of paying for OZI. The other option is for the drilling contractor to charge a normal fee to the oil extractor and determine internally what the impact of OZI is and pay OZI for usage per case. 


\section{Revenue Stream 2: License Pay Per Day Per Well Per Rig}

After reviewing the cost benefits analysis based on test on several wells during a field test. Once the value of OZI is proven, a fixed cost can be derived. The value would be based on the number of days saved. If the customer wants to use the software for 10 days, OZI will multiply the number of days by the fixed cost per day.

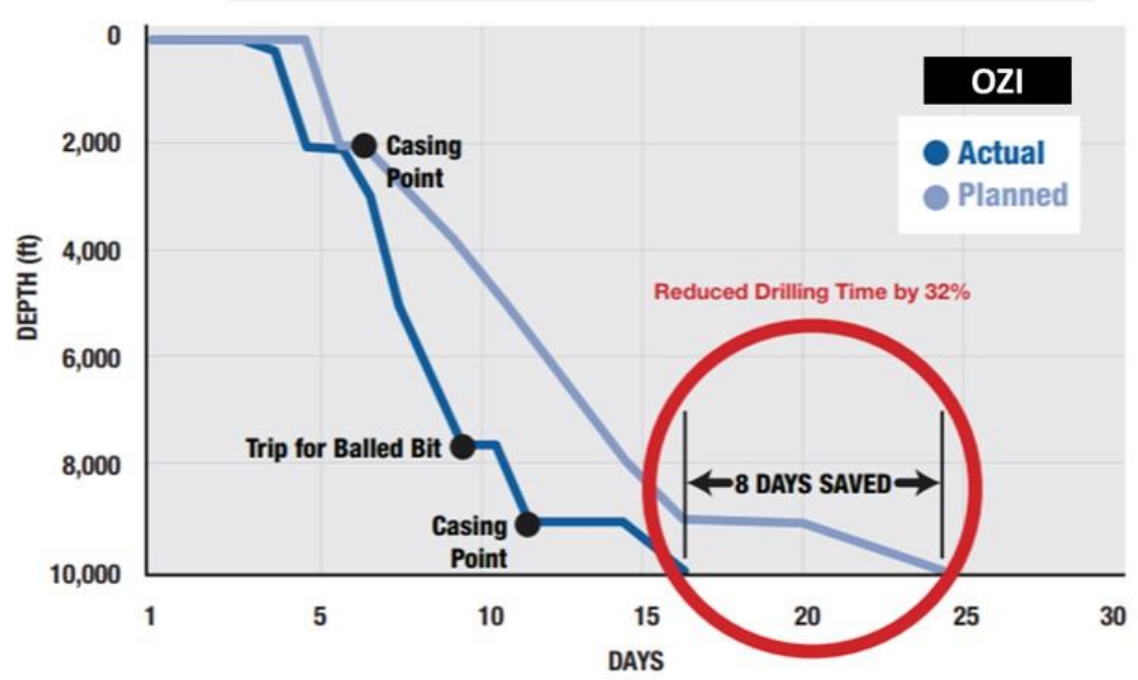

Figure 67 Time Saving Potential of OZI

OZI will also add insurance fees just in case the software is damaged or corrupted during the use of that customer. OZI will charge a little lower if the customer wants to use the product on a per well basis by assuming a standard number of wells. On a per rig basis, the cost would be much higher but some restrictions have to be in place especially timeline of usage.

\subsection{Understanding the Competition}

Schlumberger controls 7.0\% market share of Oil and Gas Field Services industry in Canada [69]. Their competing service is OptiDrill Real-Time Drilling Intelligence Service. Baker Hughes has an estimated 3.2\% market share in this industry. Their competing service is INTEQ ${ }^{\mathrm{TM}}$ drilling optimization service. Weatherford International also controls $1.7 \%$ market share this same industry. Their competing product is OneSync $®$ software. Halliburton controls $8.4 \%$ market share of Mining, Oil \& Gas Machinery Manufacturing in Canada. Their competing product is Max3Di ${ }^{\mathrm{TM}}$ 
Drilling Optimization Software. Other products from other companies are Smart Driller Indicator (China National Petroleum Corporation), Real-Time Drilling Analytics (Petrolink), and SureDrill ${ }^{\mathrm{TM}}$-Optimizer system (APS Technology).

The Competitive Advantage: OZI does not require any downhole sensor nor rig staff to maintain its operation. It is of low price compared to products from competitors. Results are displayed to the driller in real time based on surface data only; this eliminates cost accrued by customers who need to rent downhole tools from competitors for similar service. There are 13 factors where OZI can be compared to its competition: Sensors downhole, Installation cost, Additional rig hardware, Field test failure cost, Number of drilling parameters optimized, Price of Product, Tripping Time, Maintenance cost, Business liability, Speed of making decision, Drilling company's driller on payroll, Sales through the drilling company, and New market segment: oilfield waste management companies. Below is a table showing the competitor analysis between OZI and its competitors in terms of these 13 factors. 
Table 6 Competitive Analysis Table for OZI

\begin{tabular}{|c|l|l|l|l|l|l|}
\hline & $\begin{array}{c}\text { OptiDrill } \\
\text { Real-Time } \\
\text { Drilling } \\
\text { Intelligence } \\
\text { Service }\end{array}$ & $\begin{array}{c}\text { INTEQ } \\
\text { drilling } \\
\text { optimization } \\
\text { service }\end{array}$ & $\begin{array}{c}\text { OneSync® } \\
\text { software }\end{array}$ & $\begin{array}{c}\text { Max3DiTM } \\
\text { Drilling } \\
\text { Optimization } \\
\text { Software }\end{array}$ & $\begin{array}{c}\text { SureDrillTM_ } \\
\text { Optimizer } \\
\text { system }\end{array}$ & $\begin{array}{c}\text { Optimum } \\
\text { Zone } \\
\text { Identifier } \\
\text { (OZI) }\end{array}$ \\
\hline Sensors downhole & High & High & Low & Medium & High & Zero \\
\hline Installation cost & High & High & Low & Low & Low & Zero \\
\hline $\begin{array}{c}\text { Additional rig } \\
\text { hardware }\end{array}$ & High & High & Low & Medium & Medium & Zero \\
\hline Field test failure cost & High & High & Medium & Medium & Medium & Zero \\
\hline $\begin{array}{c}\text { Number of drilling } \\
\text { parameters } \\
\text { optimized }\end{array}$ & High & High & High & High & Medium & Low \\
\hline Price of Product & High & High & Medium & High & Medium & Medium \\
\hline $\begin{array}{c}\text { Tripping Time } \\
\text { Maintenance cost }\end{array}$ & Hedium & Medium & Low & Low & Low & Low \\
\hline Business liability & Medium & Medium & Low & Low & Medium & Low \\
\hline $\begin{array}{c}\text { Speed of making } \\
\text { decision }\end{array}$ & Low & Low & Low & Low & Medium & Zero \\
\hline $\begin{array}{c}\text { Drilling company's } \\
\text { driller on payroll }\end{array}$ & Zero & Zero & Zero & Zero & Zero & Medium \\
\hline $\begin{array}{c}\text { Sales through the } \\
\text { drilling company }\end{array}$ & Zero & Zero & Zero & Zero & Zero & High \\
\hline $\begin{array}{c}\text { New: oilfield waste } \\
\text { management } \\
\text { companies }\end{array}$ & Zero & Zero & Zero & Zero & Zero & High \\
\hline
\end{tabular}

\subsubsection{Strategy Canvas}

The strategy canvas was developed by W. Chan Kim and Renée Mauborgne so that a startup can understand how factors affecting a particular market are utilized by the competition; in order to carve out a path that automatically reduces the competition and creates a separate market segment for the startup [75]. It is a graphical picture of the strategies most companies are using and what move a new organization can make to standout with higher quality and newer business technology. 


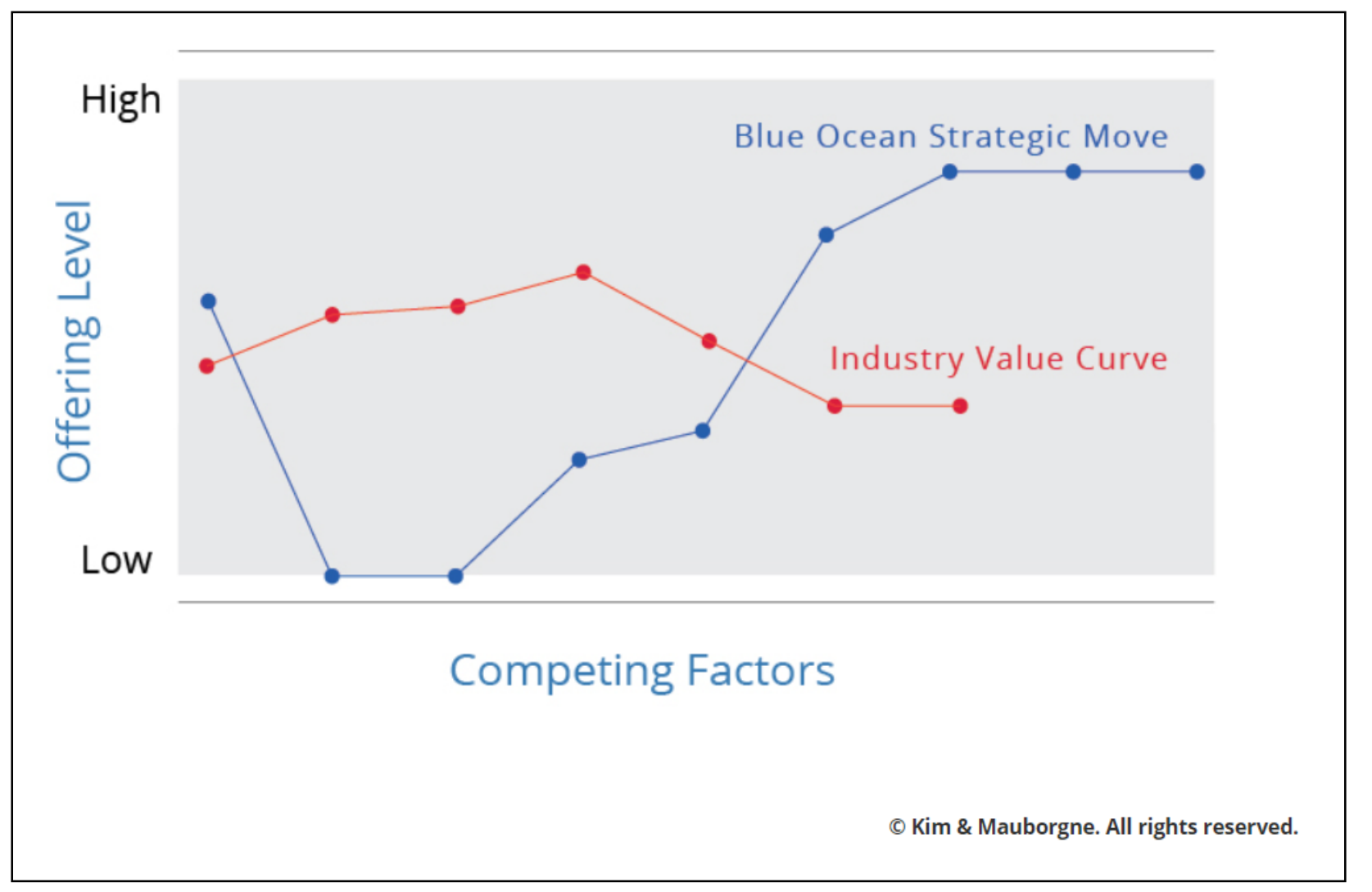

\section{Figure 68 The Blue Ocean Move [75]}

The horizontal axis is the list of competing factors that directly affect choices in the industry and the vertical axis is the offering level each competing organization strategically chooses based on its goals and vision to remain competitive [75].

The strategy canvas for OZI can be constructed as shown in the Figure 69. 


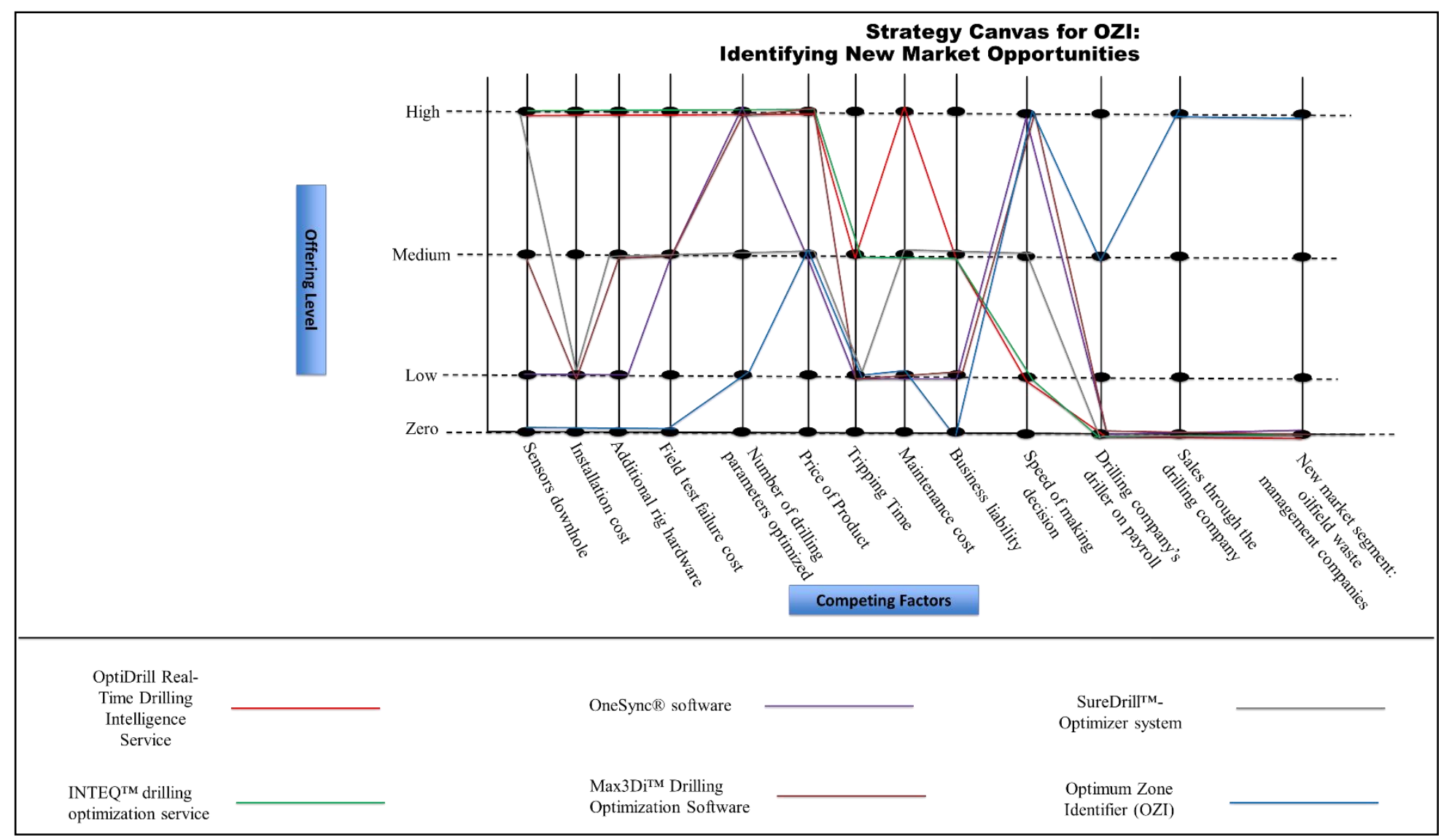

Figure 69 Blue Ocean Move for OZI

\subsubsection{Blue Ocean Strategy}

W. Chan Kim \& Renée Mauborgne came up with the terms red and blue ocean. The red ocean is the pathway the industry is moving at. This is where the competition is heavy. The blue ocean is the differentiating path which ensures a new organization avoid as much competition as much as possible [75]. The Figure below shows how management in these sets of strategies think 


\section{Break the value-cost trade-off.}

Align the whole system of a firm's activities with its strategic choice of differentiation or low cost.

Align the whole system of a firm's activities in pursuit of differentiation and low cost.

Figure 70 Blue Ocean Strategy versus Red Ocean Strategy [75]

Like the 'blue' ocean, it is vast, deep and powerful -in terms of opportunity and profitable growth. Figure 71 shows the blue ocean strategy for OZI.

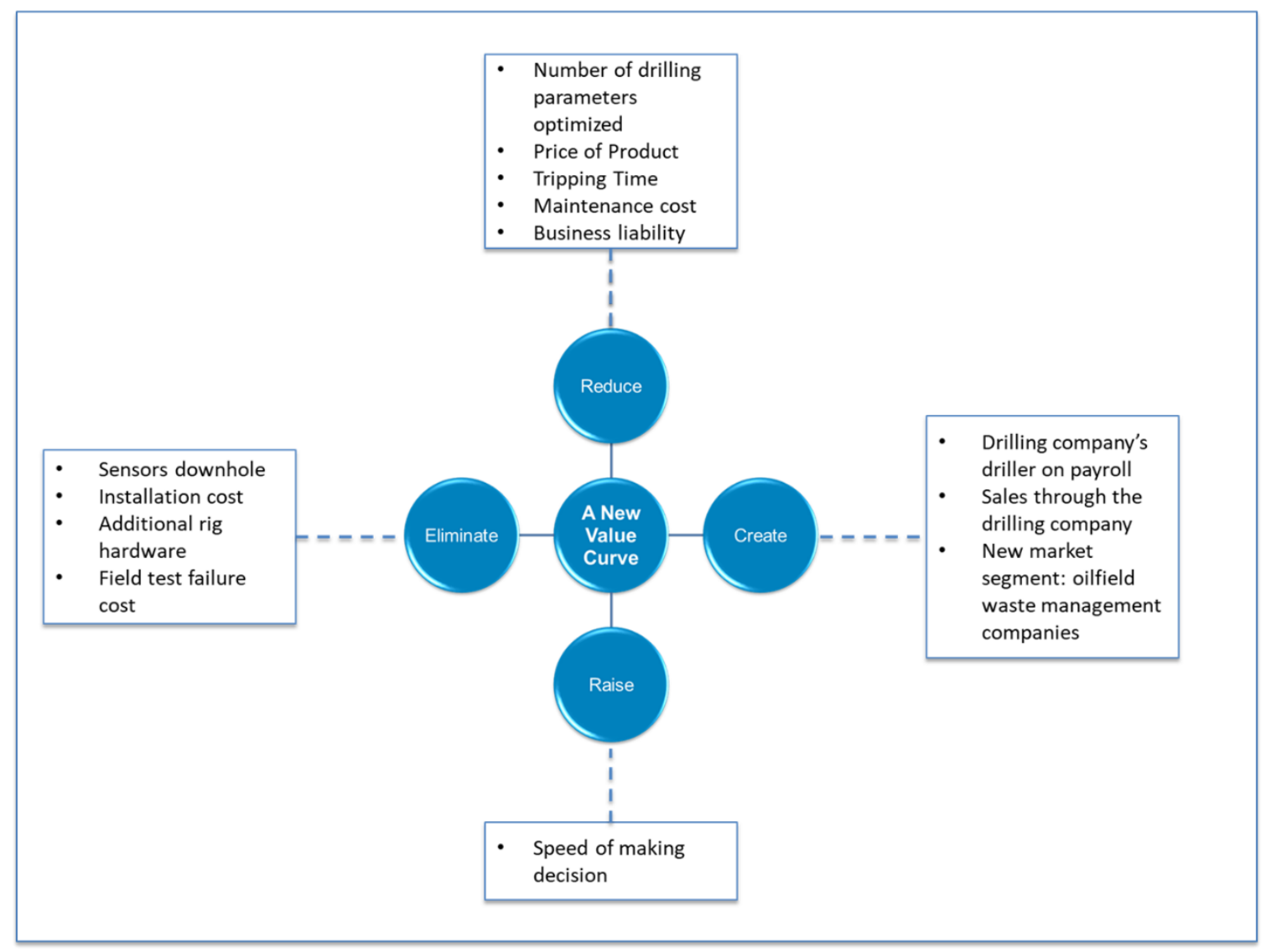

Figure 71 Blue Ocean Strategy for OZI 


\subsubsection{Perceptual/Position Map}

A perceptual map is a visualization tool that helps entrepreneurs understand the perception of the consumer about competing products. This perception shows what is in the mind of the buyer just before making that decision to purchase a product or service [76]. This map is commonly presented as a X-Y graph. When all competing products are placed on this map, it would be obvious where a new product can compete easily in the market [76]. Figure 72 shows the perceptual map for OZI.

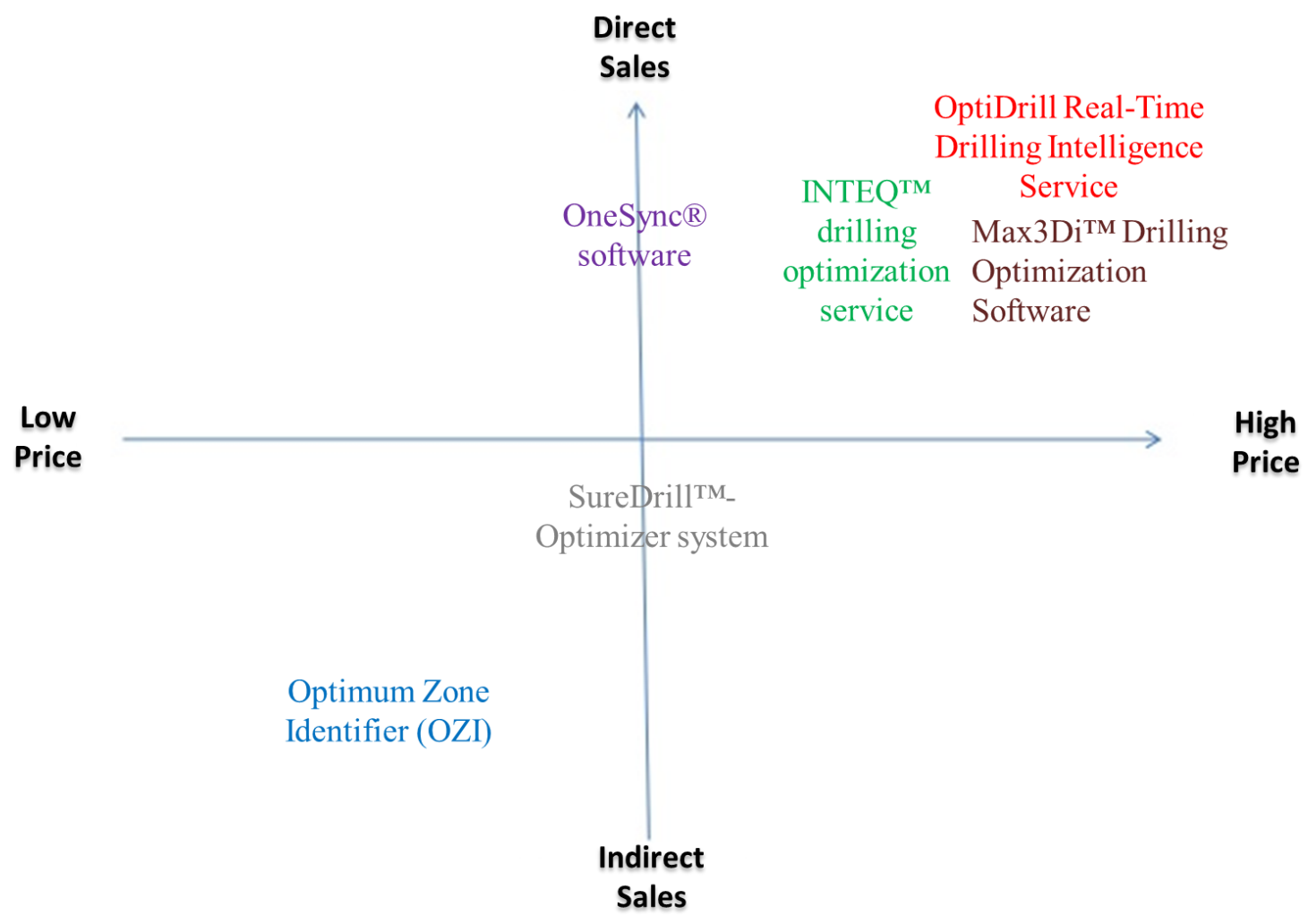

Figure 72 Perceptual Map for OZI

\subsection{Chapter Six Conclusion}

This research has potentials for running as a startup and over 70 customer discovery and customer validation interviews have been conducted to better understand the feasibility. The adoption life cycle revealed that drilling contractors would be the technology enthusiast who can give the product from this research a fair chance. The value proposition is to help customers reduce drilling operation time and optimize decision making process to reduce drill string vibrations. 


\section{Chapter Seven: CONCLUSIONS AND RECOMMENDATIONS}

This chapter talks about the summary of the technical and business processes encountered in this research and summarizes the findings from both the technical and the business study. Recommendations are made regarding what steps need to be taken next on the technical and business parts.

\subsection{Conclusions from the Technical Part}

Based on previous researcher's work, it was determined that the optimum zone chart can show the driller what drilling parameter combinations are prone to vibration zone effects. This research designed a machine learning process that helps create the optimum zone chart based on real time surface data. The steps in the entire process is outlined below.

- Collate real time surface data from the electronic drilling recorder or from the rig's operating system.

- Run the data through the optimum zone decision tree classification

- Run the data through principal component analysis and reduce the dimensions based on the capture of at least $98 \%$ significance of the original data. Go to step 2 after this.

- Use K-means clustering to pick the centroid of the optimum zone

- Use quantitative risk analysis to determine if some data in the optimum zone are at risk of moving into the optimum zone

- For each stand of pipe, the optimum zone is updated every 3.4 minutes

- When compared with downhole data recording of vibration occurrence, the optimum zone chart captures the bulk of the data and in cases of good drilling, the data falls within the optimum zone. During vibration, some data recorded as having vibrations are still in the optimum zone while all the good drilling data are in the optimum zone.

Figure 73 shows a summary of the steps taken to get to the optimum zone. 


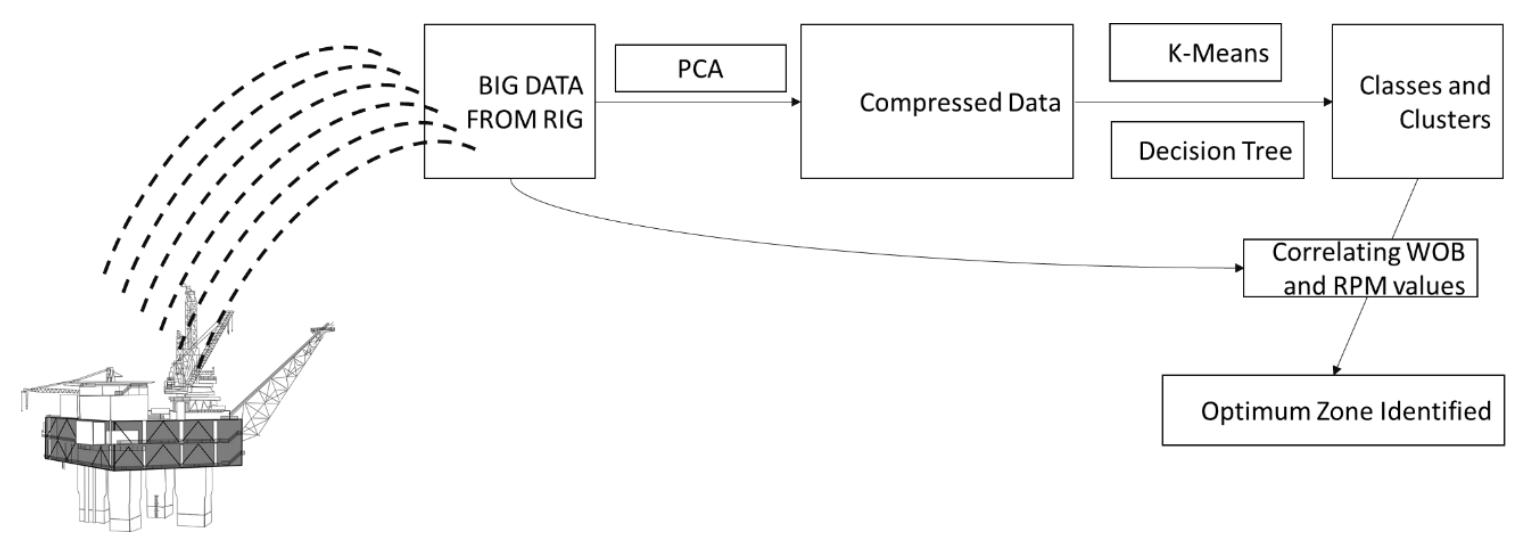

Figure 73 Developing the Optimum Zone

\subsection{Conclusions from the Business Part}

Given the uniqueness of this research, there is a potential to push for a startup. However, the need to understand the market and competition arose. Just over 70 customer discovery and customer validation interviews have been conducted. It was concluded that if the research is packaged as a software, the drilling contractor would also be the first customer and field test partner.

Below are the steps that have been taken to help encourage the IP owners of this research that there is a potential for business.

- Attendance at IADC/SPE Drilling Conference and Exhibition 2017 (The Hague, The Netherlands) which revealed that the drilling industry needs machine learning tools to solve vibration problems

- Participation at SPE Student paper contest hosted by UBC in Vancouver 2017: Victory in the Masters division showed that key industry professionals have interest in this kind of technology.

- Customer discovery interviews organized by Innovate Calgary and Hunter Centre for Entrepreneurship and Innovation for communication with potential customers showed that the pain exist which is to reduce cost and improve drilling efficiency. Victory at the final 
presentations as "Best Customer Discover Spring 2017" was a validation that the judges felt the customers for the products do exist.

- Customer validation interviews gave an opportunity for deeper talk with potential customers which today has led to partnership for field test in the summer of 2018.

- File for provisional patent through Innovate Calgary and published a paper at the SPE Annual Technical Conference and Exhibition 2018 San Antonio Texas USA.

- Participation in ENTI 781 (Introduction to Entrepreneurship) and ENTI 785 (Venture Development) helped develop the business framework the product of this research will fall into. The $5^{\text {th }}$ version of the business model canvas revealed that drilling contractors are not just the end users of this product but they are the early adopters as well.

Figure 74 shows the minimum viable product (MVP) which was created based on teachings from both ENTI classes. This is the third version of the MVP.

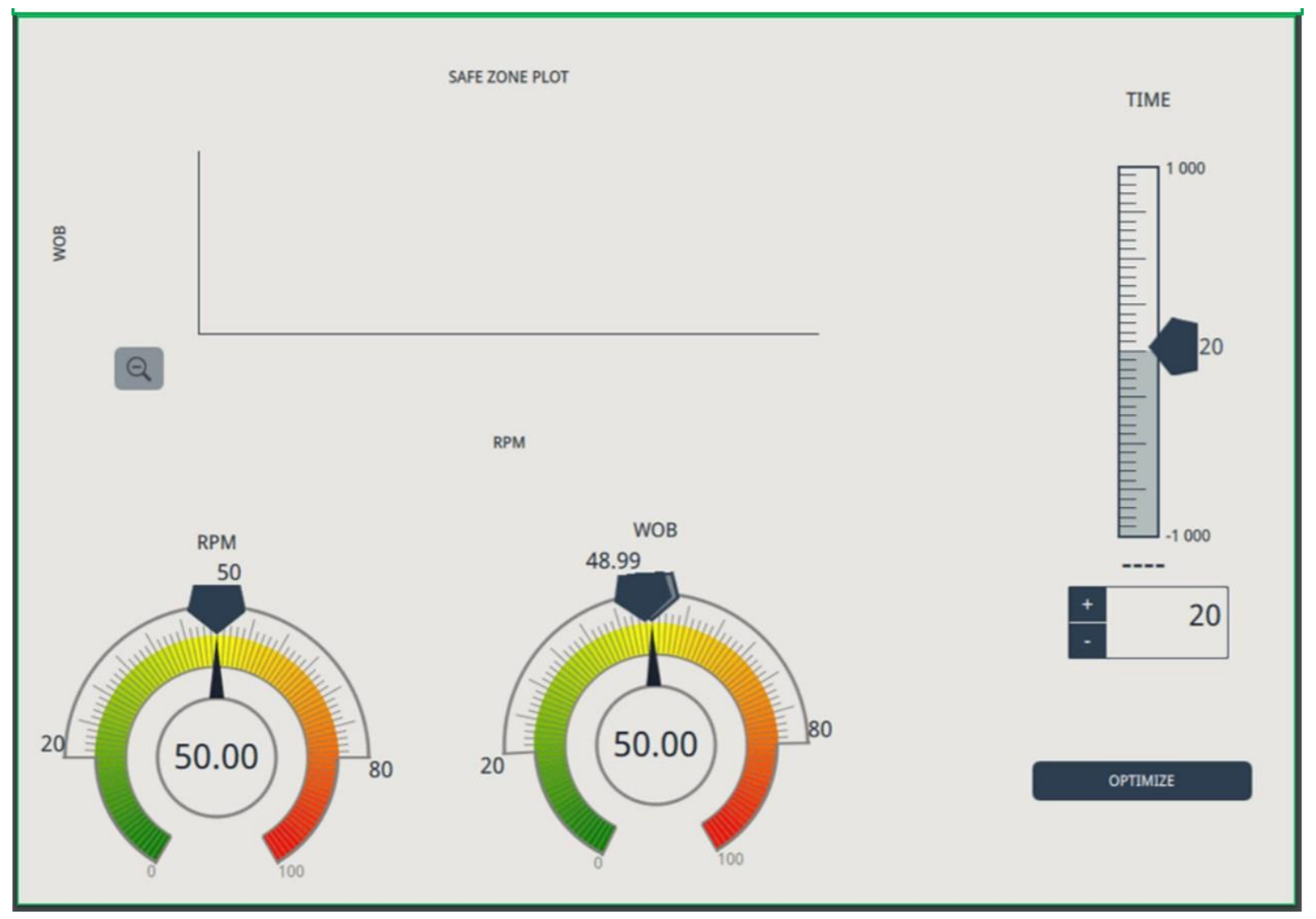

Figure 74 OZI Minimum Viable Product 


\subsection{Recommendations for the Technical Part}

The work done so far shows more positive progress towards achieving a machine learning process that identifies an optimum zone chart which completely agrees with downhole recordings of vibration measurement. However, a lot still must be done to get to the $100 \%$ accuracy. The list below shows the next steps that should be taken when further research on this topic is done.

- Achieve a distinct separation of vibration data points from good drilling data points based on downhole vibration measurement and show this distinction on the WOB/RPM plot.

- Figure out how change in rock hardness affects the optimum zone chart

- Run the optimum zone chart in such a way that each optimum zone has an impact on the next optimum zone chart and note how this affects the correlation of downhole recordings with the display on the chart

- Create a real-time scenario where vibration can be detected and combated and check to see if this affect downhole vibration measurements

- Redesign the decision tree model to become more flexible with new patterns discovered in the input data

- Relate the optimum zone chart to how ROP can be increased without causing any damage to tools downhole

\subsection{Recommendations for the Business Part}

So far, a lot of hypothesis about the business model are in the validation phase however in order to excellently validate the model, more interviews with customers need to be done. This would ensure that the exact path to capture the customer's full interest is followed and no wrong assumptions are made about the customer's behavior pattern. Another important step is to ensure the field test is actualized in the Summer of 2018. The test should be done on several wells so that lessons learnt can be immediately applied on each new well. After a successful test, the product should be taken through a branding process. What kind of brand will appeal to the hearts and minds 
of potential customers and what kind of proposal packages are eye catching to the business channels and customers? These and many more would be answered if proper branding is initiated for the Optimum Zone Identifier product. With these structures in place, the product can be formally launched to the market (full patent application should have been done before the first public announcement). Incorporating the new software company is necessary as strategies to launch the product in the Canadian, Arabian, European and Chinese markets are in place. The Figure 75 shows the summary of the next steps recommended for this product to make its first sale.

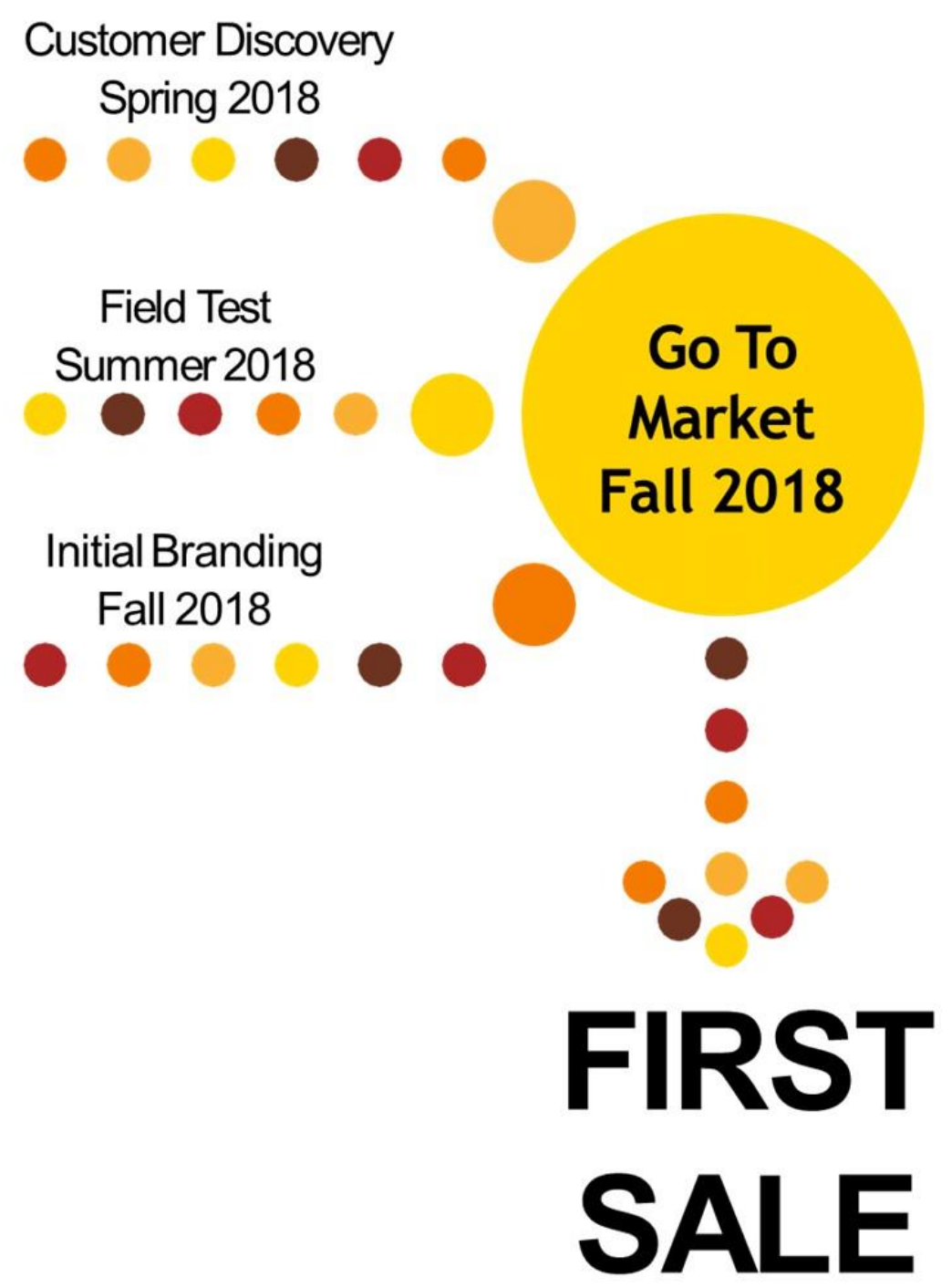

Figure 75 Early Milestones for OZI 


\subsection{Chapter Seven Conclusion}

Significant progress has been made towards identifying the optimum zone in real time. A business model that suggests a potential business for this research has been proposed. There is more work to do in the research but the field test in the summer of 2018 will give the true validity of this research. A positive result will attract drilling contractors, oil extractors and oilfield waste management companies. 


\section{REFERENCES}

1. Shor R.J, “ENCH 689 Lecture Notes 2018” Winter Term

2. Thonhauser, S. G., \& Leoben, M. (2009). Introduction to Drilling Engineering and Well Design

3. Drillstring Vibrations and Vibration Modeling - Schlumberger. http://www.slb.com/ /media/Files/drilling/brochures/drilling_opt/drillstring_vib_br.ashx

4. Junor, Duncan J. (2007), “Challenges and developments in direct measurement of down hole forces affecting drilling efficiency”, MSc thesis, Robert Gordon University, Aberdeen.

5. Zhua Q., Zou Z., Huangc B., Maa L., Xiad J., (March 2017). Downhole vibration causing a drill collar failure and solutions. Natural Gas Industry B: Volume 4, Issue 2

6. Larsen L.K. (2014). Tools and Techniques to Minimize Shock and Vibration to the Bottom Hole Assembly. University of Stavanger

7. Gulyayev V.I., L. V. Shevchuk L.V. (2015) " Drill String Bit Whirl Simulation With the Use of Frictional and Nonholonomic Models " Journal of Vibration and Acoustics | Volume 138 | Issue 1 | research-article

8. W.D. Aldred, M.C. Sheppard (1992), "Drillstring vibrations: a new generation mechanism and control strategies", Proceedings of the 67th Annual Technical Conference and Exhibition of SPE, Washington, DC.

9. H. Hakimi,S. Moradi (2010) "Drillstring vibration analysis using differential quadrature method" Journal of Petroleum Science and Engineering

10. Challamel, N., Sellami, H., Chenevez, E., \& Gossuin, L. (2000, January 1). A Stick-slip Analysis Based on Rock/Bit Interaction: Theoretical and Experimental Contribution. Society of Petroleum Engineers. doi:10.2118/59230-MS

11. Forster, I. (2011, January 1). Axial Excitation as a Means of Stick Slip Mitigation - Small Scale Rig Testing and Full Scale Field Testing. Society of Petroleum Engineers. doi:10.2118/139830-MS

12. Zakuan, A., Abd Aziz, A. J., Subroto, B., Hermawan, H., Othman, A. F., Shariff, A. H. B., \& Jahari, S. A. (2011, January 1). Stick Slip Mitigation Plan to Improve Drilling. Society of Petroleum Engineers. doi:10.2118/141988-MS

13. Schwefe, T., Ledgerwood III, L. W., Jain, J. R., Fuselier, D. M., Oueslati, H., \& Endres, L. (2014, March 4). Development and Testing of Stick/Slip-Resistant PDC Bits. Society of Petroleum Engineers. doi:10.2118/168026-MS 
14. Efteland, F., Creegan, A., Jordan, L., \& Caraway, C. (2015, November 9). The Significance of Proactive Online Monitoring with Stick-slip Mitigation. Society of Petroleum Engineers. doi:10.2118/177950-MS

15. Vogel, S. K., \& Creegan, A. P. (2016, January 26). Case Study for Real Time Stick/Slip Mitigation to Improve Drilling Performance. Society of Petroleum Engineers. doi:10.2118/178176-MS

16. Shen, Y., Zhang, Z., Zhao, J., Chen, W., Hamzah, M., Harmer, R., \& Downton, G. (2017, October 9). The Origin and Mechanism of Severe Stick-Slip. Society of Petroleum Engineers. doi:10.2118/187457-MS

17. Bailey, J. R., Payette, G. S., \& Wang, L. (2018, March 6). Improved Methods to Understand and Mitigate Stick-Slip Torsional Vibrations. Society of Petroleum Engineers. doi:10.2118/189673-MS

18. Vandiver, J. K., Nicholson, J. W., \& Shyu, R.-J. (1989, January 1). Case Studies of the Bending Vibration and Whirling Motion of Drill Collars. Society of Petroleum Engineers. doi:10.2118/18652-MS

19. Zhang, Y., \& Xiao, Z. (1992, January 1). Lateral Vibration And Bending Stress Of A Joint Of Drill Pipe Under Self-Excited Whirling. Society of Petroleum Engineers.

20. Rey-Fabret, I., Mabile, M. C., \& Oudin, N. (1997, January 1). Detecting Whirling Behaviour of the Drill String From Surface Measurements. Society of Petroleum Engineers. doi:10.2118/38587-MS

21. Hutchinson, M., Burgess, D., Thompson, F., \& Kopfstein, A. (2013, September 30). Self-Adapting Bottom Hole Assembly Vibration Suppression. Society of Petroleum Engineers. doi:10.2118/166071-MS

22. Marquez, A., Omojuwa, E., \& Teodoriu, C. (2017, April 18). Mitigating Downhole Vibrations in Bottom Hole Assemblies Using Finite Element Analysis. Society of Petroleum Engineers. doi:10.2118/184420-MS

23. Zhao, J., Shen, Y., Chen, W., Zhang, Z., \& Johnston, S. (2017, October 4). Machine LearningBased Trigger Detection of Drilling Events Based on Drilling Data. Society of Petroleum Engineers. doi:10.2118/187512-MS

24. Slonneger, J. C. (1937, January 1). Vibration Problems in Oil Wells. American Petroleum Institute.

25. Bailey, J. J., \& Finnie, I. (1960). An Experimental Study of Drill-String Vibration. Journal of Engineering for Industry | Volume 82 | Issue 2 | Research Paper doi:10.1115/1.3663020 
26. Deily F.H., Dareing D.W., Paff G.H., Ortloff J.E., Lynn R.D. (1968). Downhole Measurements of Drill String Forces and Motions. Journal of Engineering for Industry | Volume 90 | Issue 2 | Research Paper. doi:10.1115/1.3604617

27. Cunningham, R. A. (1968). Analysis of downhole measurements of drill string forces and motions. Journal of Engineering for Industry | Volume 90 | Issue 2 | Research Paper. doi:10.1115/1.3604616 28. Skaugen, E. (1987). The Effects of Quasi-Random Drill Bit Vibrations Upon Drillstring Dynamic Behavior. SPE Annual Technical Conference and Exhibition, 105-116. http://doi.org/10.2118/16660-MS

29. Langeveld, C. J. (1992, January 1). PDC Bit Dynamics. Society of Petroleum Engineers. doi:10.2118/23867-MS

30. Rajnauth J.J., (2003). Reduce Torsional Vibration and Improve Drilling Operations. SPE Latin American and Caribbean Petroleum Engineering Conference, 27-30 April, Port-of-Spain, Trinidad and Tobago.

31. Craig A.D., Hanley C., McFarland B., Shearer D.R., King P.D. (2009). A Proven Approach to Mitigating Drilling Vibration Problems in offshore Western Australia. International Petroleum Technology Conference, 7-9 December, Doha, Qatar.

32. Mario B., Julio M., Martin G., Oscar R., Gerardo L., Pedro T.J. (2014). Mitigating Vibration with Roller Reamer Technology Improves Drilling Efficiency in Hard/Abrasive Formation, Sao Francisco Basin, Brazil. SPE Latin America and Caribbean Petroleum Engineering Conference, 21-23 May, Maracaibo, Venezuela.

33. Greenwood, J. A. (2016, March 1). Improvements in the Root Cause Analysis of Drill string Vibration. Society of Petroleum Engineers. doi:10.2118/178819-MS

34. Greenwood, J. A. (2016, March 1). Vibration Monitoring and Mitigation - An Integrated Measurement System. Society of Petroleum Engineers. doi:10.2118/178773-MS

35. Rajnauth, J. J. (2003, January 1). Reduce Torsional Vibration and Improve Drilling Operations. Society of Petroleum Engineers. doi:10.2118/81174-MS

36. Craig, A. D., Hanley, C., McFarland, B., Shearer, D. R., \& King, P. D. (2009, January 1). A Proven Approach to Mitigating Drilling Vibration Problems in offshore Western Australia. International Petroleum Technology Conference. doi:10.2523/IPTC-13399-MS 
37. Wu, X., Karuppiah, V., Nagaraj, M., Partin, U. T., Machado, M., Franco, M., \& Duvvuru, H. K. (2012, January 1). Identifying the Root Cause of Drilling Vibration and Stick-Slip Enables Fit-forPurpose Solutions. Society of Petroleum Engineers. doi:10.2118/151347-MS

38. Amorim, D. S., Hanley, C., \& Leite Carvalho, D. J. (2012, January 1). BHA Selection and Parameter Definition Using Vibration Prediction Software Leads to Significant Drilling Performance Improvements. Society of Petroleum Engineers. doi:10.2118/152231-MS

39. Hanley, C., Stuart, S., Bass, C., \& Garcia, A. (2012, January 1). Development of a Software System for Drilling Optimization using Surface and Downhole Drilling Data Modeling a Human Process for Drilling Optimization. Society of Petroleum Engineers. doi:10.2118/152934-MS

40. Song, P., Shi, L., Zhou, Y., Zhao, Q., Jiang, H., \& Cui, P. (2014, November 12). Study and Applications on Integrated Drilling Engineering Software for Drilling Engineering Design and Real-time Optimization. Society of Petroleum Engineers. doi:10.2118/172288-MS

41. P. Louridas and C. Ebert, "Machine Learning," in IEEE Software, vol. 33, no. 5, pp. 110-115, 2016. doi:10.1109/MS.2016.114

42. Zhang. Q., Jiarong S., (1991). The Application of Machine Learning to Rock Mechanics. 7th ISRM Congress, 16-20 September, Aachen, Germany.

43. Vladimir A., Aaron R., Karen H., Eduardo M., Justo M., Tamara L., Natasha P. (2002). Selection of EOR/IOR Opportunities Based on Machine Learning. European Petroleum Conference, 29-31 October, Aberdeen, United Kingdom.

44. Sarkar D., Osborne M., Adcock T., (2016). A Machine Learning Approach to the Prediction of Tidal Currents. The 26th International Ocean and Polar Engineering Conference, 26 June-2 July, Rhodes, Greece.

45. Bangert, P. (2017, May 9). Smart Condition Monitoring Using Machine Learning. Society of Petroleum Engineers. doi:10.2118/187936-MS

46. Alpaydin, Ethem. Introduction to Machine Learning, edited by Ethem Alpaydin, MIT Press, 2014. ProQuest Ebook Central, Created from ucalgary-ebooks on 2017-05-22 18:06:30.

47. Ben-David, S. (2014). Understanding Machine Learning : From Theory to Algorithms. Cambridge University Press.

48. Wu, X., Paez, L. C., Partin, U. T., \& Agnihotri, M. (2010, January 1). Decoupling Stick/Slip and Whirl To Achieve Breakthrough in Drilling Performance. Society of Petroleum Engineers. doi:10.2118/128767-MS 
49. Schlumberger. Unconfined Compressive Strength. Retrieved from http://www.glossary.oilfield.slb.com/Terms/u/unconfined_compressive_strength.aspx

50. Ernst, S., Pastusek, P. E., \& Lutes, P. J. (2007, January 1). Effects of RPM and ROP on PDC Bit Steerability. Society of Petroleum Engineers. doi:10.2118/105594-MS

51. Dupriest, F. E. (2006, January 1). Comprehensive Drill Rate Management Process To Maximize ROP. Society of Petroleum Engineers. doi:10.2118/102210-MS

52. Belaskie, J. P., Dunn, M. D., \& Choo, D. K. (1993, June 1). Distinct Applications of MWD, Weight on Bit, and Torque. Society of Petroleum Engineers. doi:10.2118/19968-PA

53. Rabia H. (2002). Well Engineering \& Construction. ISBN: 0954108701.

54. Boonsri, K. (2014, August 25). Torque Simulation in the Well Planning Process. Society of Petroleum Engineers. doi:10.2118/170500-MS

55. Pason. Electronic Drilling Recorder Brochure. BR005_EN_EDR_Brochure_rev1

56. National Oilwell Varco. Just Add NOVOS. Retrieved from https://www.nov.com/WorkArea/DownloadAsset.aspx?id=27496

57. National Oilwell Varco (2018). NOVOS (Reflective Drilling System). Retrieved from http://www.nov.com/NOVOS.aspx

58. Lindsay, S., 2002. A tutorial on principal component analysis. Details available at /csnet.otago.ac.nz/cosc453/student_tutorials/principal_components.pdfS, last accessed on 18 October 2007

59. Duncan Gillies \& Marc Diesenroth. DOC493: Intelligent Data Analysis and Probabilistic Inference Lecture 14. Department of Computing, Imperial College London.

60. Madhulatha T.S. (2012). An Overview on Clustering Methods. IOSR Journal of Engineering Apr. 2012, Vol. 2(4) pp: 719-725

61. Prashant Gupta (2017, May 17). Decision Trees in Machine Learning. Retrieved from https://towardsdatascience.com/decision-trees-in-machine-learning-641b9c4e8052

62. Kumar T.S. (2004). Classification: Basic Concepts Decision Trees and Model Evaluation. Retrieved from https://www-users.cs.umn.edu/ kumar001/dmbook/ch4.pdf

63. Tyler Cobb, Dan Scott, Ron Dirksen, Alfazazi Dourfaye, Craig Fleming, Richard Rivera and Jorge Silveus. (2014). Bits. IADC Drilling Manual. 12th Edition.

64. Schumberger. Drilling Optimization Workflow. Retrieved from https://www.slb.com/ /media/Files/drilling/posters/shock_vibration_posters.ashx 
65. Liang, Q. J. (2002, January 1). Application of Quantitative Risk Analysis to Pore Pressure and Fracture Gradient Prediction. Society of Petroleum Engineers. doi:10.2118/77354-MS

66. Blank S., Bob Dorf B. (2012, March 1). The Startup Owner's Manual: The Step-By-Step Guide for Building a Great Company. K \& S Ranch Publishing Division

67. Wohlers Associates, Inc. (2002). Rapid Prototyping \& Tooling State of the Industry. Wohlers Report 2002

68. Geoffrey Moore (1995). Inside the Tornado. Harper Collins Publishing.

69. IBISWorld Canada Industry Reports (2017). 21111CA Oil Drilling \& Gas Extraction.

70. Bennett Jones LLP (2016). The Upstream Oilfield Services Industry in Western Canada, A Backgrounder.

71. Alexander Osterwalder (2012). Achieve Product-Market Fit with Our Brand-New Value Proposition Designer Canvas. Retrieved from http://businessmodelalchemist.com/blog/2012/08/achieve-product-market-fit-with-our-brand-newvalue-proposition-designer.html

72. Stategyzer AG. The Value Proposition Canvas. Retrieved from https://strategyzer.com/canvas/value-proposition-canvas

73. Osterwalder A. (2004). The Business Model Ontology - A Proposition in a Design Science Approach. The University of Lausanne in Lausanne, Switzerland

74. Stategyzer AG. The Business Model Canvas. Retrieved from https://strategyzer.com/canvas/business-model-canvas

75. Kim W.C., Mauborgne R. (2005, Febuary 1). Blue Ocean Strategy: How To Create Uncontested Market Space And Make The Competition Irrelevant. Harvard Business School Press

76. Fripp G. The Perceptual Process. Retrieved from http://www.perceptualmaps.com/understandingperceptual-maps/the-perceptual-process/

77. Federick Colorado. What are the factors for selection of oil and gas well drill sites? Retrieved from http://www.frederickco.gov/435/FAQ---Selection-of-Oil-Gas-Well-Drill-Si

78. PetroWiki. Borehole Instability. Retrieved from http://petrowiki.org/Borehole_instability

79. Saldivar B. Boussaada I. Mounier H. Mondi'e S. Niculescu S.I. (August 24-29,2014). An Overview on the Modeling of Oilwell Drilling Vibrations. Proceedings of the 19th World Congress The International Federation of Automatic Control Cape Town, South Africa 
80. Runia, D. J., Dwars, S., \& Stulemeijer, I. P. J. M. (2013, March 5). A brief history of the Shell "Soft Torque Rotary System" and some recent case studies. Society of Petroleum Engineers. doi:10.2118/163548-MS

81. Kyllingstad, A., \& Nessjøen, P. J. (2009, January 1). A New Stick-Slip Prevention System. Society of Petroleum Engineers. doi:10.2118/119660-MS

82. Dwars, S. (2015, March 17). Recent Advances in Soft Torque Rotary Systems. Society of Petroleum Engineers. doi:10.2118/173037-MS

83. Al Dushaishi M., Nygaard R., Hoel E., Andersen E., \& Hellvik S. (May 2015). Post Well Vibration Analysis in the North Sea: A Tool to Understand Drilling Performance. ASME 2015 34th International Conference on Ocean, Offshore and Arctic Engineering.

84. Paes, P., Ajikobi, F., \& Chen, D. .-K. (2005, January 1). Challenges in Drilling in Campos Basin. Society of Petroleum Engineers. doi:10.2118/95418-MS

85. Baumgartner, T., \& van Oort, E. (2014, October 27). Pure and Coupled Drill String Vibration Pattern Recognition in High Frequency Downhole Data. Society of Petroleum Engineers. doi:10.2118/170955-MS

86. Trevino A. (2016). Introduction to Kmeans Clustering. Retrieved from https://www.datascience.com/blog/k-means-clustering 


\section{APPENDIX I: Matlab Code for Optimum Zone/Safe Zone Chart}

1. Identifying variables

data $=\operatorname{load}($ 'ChangeLog.txt');

$\mathrm{RPM}=\operatorname{data}(:, 1)$;

$\mathrm{ROP}=\operatorname{data}(:, 2)$;

$\mathrm{WOB}=$ data $(:, 3)$;

$\mathrm{BD}=\operatorname{data}(:, 4)$;

DMEA=data $(:, 5)$;

TIME=data $(:, 6)$;

2. Calculating the Principal Components

[coeff,score,latent,tsquared,explained $]=$ pca $($ data $)$

mapcaplot(data)

cumsum(latent)./sum(latent)

3. Plotting Principal Components and comparing their variances (level of significance)

figure()

$\operatorname{plot}\left(\operatorname{score}(:, 1), \operatorname{score}(:, 2),{ }^{\prime}+\right.$ ')

'axes square'

xlabel('1st Principal Component')

ylabel('2nd Principal Component')

$[\mathrm{pc}]=\operatorname{pca}($ data $)$

4. Explained means the level of significance of each principal component

figure()

pareto(explained)

xlabel('Principal Component')

ylabel('Variance Explained (\%)')

5. Score is the new value of the data points when plotted with the principal components as the axis instead of the original $X$ and $Y$ coordinate system

figure()

scatter3(score $(:, 1)$, score $(:, 2)$, score $(:, 3))$

axis equal

xlabel('1st Principal Component')

ylabel('2nd Principal Component')

zlabel('3rd Principal Component')

6. Comparing the depth of the bit and the depth of the hole while drilling

figure()

plot(TIME,RPM)

figure()

plot(TIME,BD)

hold on

plot(TIME,DMEA) 
title('Depth and Time Plot');

ylabel('Bid Depth and Hole Depth');

xlabel('Time')

legend ('BD','DMEA')

hold off

7. Calculating the upper and lower limits for WOB and RPM

$\mathrm{P}=1.157625^{*}$ mean(WOB);

$\mathrm{Q}=0.729 *$ mean $(\mathrm{WOB})$;

$\mathrm{R}=0.729 *$ mean $(\mathrm{RPM})$;

$\mathrm{S}=1.331$ *mean $(\mathrm{RPM})$;

8. Isolating the data points for each zone so that analysis can be done on each zone

StickSlip $=[\mathrm{RPM}(\mathrm{WOB}>\mathrm{P}), \mathrm{WOB}(\mathrm{WOB}>\mathrm{P})]$;

ForwardWhirling $=[\mathrm{RPM}(\mathrm{WOB}<\mathrm{Q}), \mathrm{WOB}(\mathrm{WOB}<\mathrm{Q})]$;

LowROP $=[\mathrm{RPM}((\mathrm{RPM}<\mathrm{R}) \&(\mathrm{WOB}<\mathrm{P}) \&(\mathrm{WOB}>\mathrm{Q})), \mathrm{WOB}((\mathrm{RPM}<\mathrm{R}) \&(\mathrm{WOB}<\mathrm{P}) \&(\mathrm{WOB}>\mathrm{Q}))]$ ;

BackwardWhirling $=[\mathrm{RPM}((\mathrm{RPM}>\mathrm{S}) \&(\mathrm{WOB}<\mathrm{P}) \&(\mathrm{WOB}>\mathrm{Q})), \mathrm{WOB}((\mathrm{RPM}>\mathrm{S}) \&(\mathrm{WOB}<\mathrm{P}) \&(\mathrm{~W}$ OB>Q))];

OptimumZone $=[\mathrm{RPM}((\mathrm{WOB}<\mathrm{P}) \&(\mathrm{WOB}>\mathrm{Q}) \&(\mathrm{RPM}>\mathrm{R}) \&(\mathrm{RPM}<\mathrm{S})), \mathrm{WOB}((\mathrm{WOB}<\mathrm{P}) \&(\mathrm{WOB}$ $>\mathrm{Q}) \&(\mathrm{RPM}>\mathrm{R}) \&(\mathrm{RPM}<\mathrm{S}))]$;

9. Do Reverse PCA to get original data (excluding principal components whose percentages don't add up to the first $98 \%$ representation of the original data)

$\mathrm{M}=$ mean(data);

[eigenvectors $]=\operatorname{pca}($ data $)$;

eigenvectors $(:, 9)=0$;

eigenvectors $(:, 8)=0$;

eigenvectors $(:, 7)=0$;

eigenvectors $(:, 6)=0$;

eigenvectors $(:, 5)=0$;

eigenvectors $(:, 4)=0$;

eigenvectors $(:, 3)=0$;

eigenvectors $(:, 2)=0$;

A=eigenvectors';

$\mathrm{B}=\mathrm{score}^{*} \mathrm{~A}$;

$\mathrm{N}=\mathrm{B}(:, 1)+\mathrm{M}(1,1)$;

$\mathrm{W}=\mathrm{B}(:, 3)+\mathrm{M}(1,3)$;

10. Calculating the upper and lower limits for WOB and RPM after PCA has been applied

$\mathrm{Pa}=1.157625 * \operatorname{mean}(\mathrm{W})$;

$\mathrm{Qa}=0.729 * \operatorname{mean}(\mathrm{W})$;

$\mathrm{Ra}=0.729 * \operatorname{mean}(\mathrm{N})$;

$\mathrm{Sa}=1.331 * \operatorname{mean}(\mathrm{N})$; 
11. Isolating the data points for each zone so that analysis can be done on each zone after PCA has been done

newStickSlip $=[\mathrm{N}(\mathrm{W}>\mathrm{Pa}), \mathrm{W}(\mathrm{W}>\mathrm{Pa})]$;

newForwardWhirling $=[\mathrm{N}(\mathrm{W}<\mathrm{Qa}), \mathrm{W}(\mathrm{W}<\mathrm{Qa})]$;

newLowROP=[N((N<Ra)\& $(\mathrm{W}<\mathrm{Pa}) \&(\mathrm{~W}>\mathrm{Qa})), \mathrm{W}((\mathrm{N}<\mathrm{Ra}) \&(\mathrm{~W}<\mathrm{Pa}) \&(\mathrm{~W}>\mathrm{Qa}))]$;

newBackwardWhirling $=[\mathrm{N}((\mathrm{N}>\mathrm{Sa}) \&(\mathrm{~W}<\mathrm{Pa}) \&(\mathrm{~W}>\mathrm{Qa})), \mathrm{W}((\mathrm{N}>\mathrm{Sa}) \&(\mathrm{~W}<\mathrm{Pa}) \&(\mathrm{~W}>\mathrm{Qa}))]$;

newOptimumZone $=[\mathrm{N}((\mathrm{W}<\mathrm{Pa}) \&(\mathrm{~W}>\mathrm{Qa}) \&(\mathrm{~N}>\mathrm{Ra}) \&(\mathrm{~N}<\mathrm{Sa})), \mathrm{W}((\mathrm{W}<\mathrm{Pa}) \&(\mathrm{~W}>\mathrm{Qa}) \&(\mathrm{~N}>\mathrm{Ra}) \&($

$\mathrm{N}<\mathrm{Sa}))]$;

12. Computing the risk of data points falling into vibration zones using quantitative risk analysis (this is done before PCA is applied)

a. For Stick Slip Zone

$\mathrm{SOa}=$ mean(StickSlip)-mean(OptimumZone);

$\mathrm{SOb}=\operatorname{sqrt}\left(\left((\operatorname{std}(\right.\right.$ StickSlip $\left.)) .^{\wedge} 2\right)+\left((\operatorname{std}(\right.$ OptimumZone $\left.\left.)) .^{\wedge} 2\right)\right)$;

$\mathrm{SO}=\mathrm{abs}(\mathrm{SOa} / \mathrm{SOb})$;

$\mathrm{RSO}=0.8 * \mathrm{SO}$;

b. For Forward Whirling Zone

$\mathrm{OFa}=$ mean (OptimumZone)-mean(ForwardWhirling);

$\mathrm{OFb}=\operatorname{sqrt}\left(\left((\operatorname{std}(\right.\right.$ OptimumZone $\left.)) .{ }^{\wedge} 2\right)+((\operatorname{std}($ ForwardWhirling $\left.)) . \wedge 2)\right)$;

$\mathrm{OF}=\mathrm{abs}(\mathrm{OFa} / \mathrm{OFb})$;

$\mathrm{ROF}=0.8 * \mathrm{OF}$;

c. For Backward Whirling Zone

$\mathrm{BOa}=$ mean (BackwardWhirling)-mean(OptimumZone);

$\mathrm{BOb}=\operatorname{sqrt}\left(\left((\operatorname{std}(\right.\right.$ BackwardWhirling $\left.)) .^{\wedge} 2\right)+\left((\operatorname{std}(\right.$ OptimumZone $\left.\left.)) .^{\wedge} 2\right)\right)$;

$\mathrm{BO}=\mathrm{abs}(\mathrm{BOa} / \mathrm{BOb})$;

$\mathrm{RBO}=0.8^{*} \mathrm{BO}$;

13. Computing the coordinates of the safe zone polygon

$\mathrm{Pn}=\mathrm{Pa}-\mathrm{RSO}$

$\mathrm{Sm}=((\mathrm{Sa}-\mathrm{Ra}) / 2)+\mathrm{Ra}$;

$\mathrm{Qn}=\mathrm{Qa}+\mathrm{ROF}$;

$\mathrm{Pm}=((\mathrm{Pa}-\mathrm{Qa}) / 2)+\mathrm{Qa}$;

$\mathrm{Sn}=\mathrm{Sa}-\mathrm{RBO}$;

14. Isolating the safe zone: using the basic equation formula: $y=m x+c$

a. Isolating the corner of Stick Slip and Low ROP

point $1=[\mathrm{Ra} \mathrm{Pm}]$

point2 $=[\mathrm{Sm} \mathrm{Pn}]$;

$\mathrm{m} 1=(\mathrm{Pn}-\mathrm{Pm}) /(\mathrm{Sm}-\mathrm{Ra})$;

$\mathrm{c} 1=\mathrm{Pn}-\mathrm{m} 1 * \mathrm{Sm}$;

$\mathrm{y} 1=\left(\mathrm{N}^{*} \mathrm{~m} 1\right)+\mathrm{c} 1$;

Safe1 $=[\mathbf{N}(\mathrm{W}<\mathrm{y} 1), \mathrm{W}(\mathrm{W}<\mathrm{y} 1)]$; 
b. Isolating the corner of Backward Whirling and Forward Whirling

point3=[Sm Qn];

point $4=[\mathrm{Sn} \mathrm{Pm}]$;

$\mathrm{m} 2=(\mathrm{Pm}-\mathrm{Qn}) /(\mathrm{Sn}-\mathrm{Sm})$;

$\mathrm{c} 2=\mathrm{Pm}-\mathrm{m} 2 * \mathrm{Sn}$;

$\mathrm{y} 2=(\mathrm{N} * \mathrm{~m} 2)+\mathrm{c} 2$;

Safe2 $=[N(W>y 2), W(W>y 2)]$;

c. Isolating the corner of Low ROP and Forward Whirling

point5=[Ra Pm $]$;

point6 $=[\mathrm{Sm} \mathrm{Qn}]$;

$\mathrm{m} 3=(\mathrm{Qn}-\mathrm{Pm}) /(\mathrm{Sm}-\mathrm{Ra})$;

c3 $=\mathrm{Qn}-\mathrm{m} 3 * \mathrm{Sm}$;

$\mathrm{y} 3=\left(\mathrm{N}^{*} \mathrm{~m} 3\right)+\mathrm{c} 3$;

Safe3 $=[N(W>y 3), W(W>y 3)]$;

d. Isolating the corner of Stick Slip and Backward Whirling

point $7=[\mathrm{Sm} \mathrm{Pn}]$;

point $8=[\mathrm{Sn} \mathrm{Pm}]$;

$\mathrm{m} 4=(\mathrm{Pm}-\mathrm{Pn}) /(\mathrm{Sn}-\mathrm{Sm})$;

$\mathrm{c} 4=\mathrm{Pm}-\mathrm{m} 4 * \mathrm{Sn}$;

$\mathrm{y} 4=(\mathrm{N} * \mathrm{~m} 4)+\mathrm{c} 4$;

Safe4 $=[N(W<y 4), W(W<y 4)]$;

15. Capturing data points in the safe zone (by combing the formulas for isolating all the vibration prone corners

SafeZone $=[\mathrm{N}((\mathrm{W}<\mathrm{y} 1) \&(\mathrm{~W}>\mathrm{y} 2) \&(\mathrm{~W}>\mathrm{y} 3) \&(\mathrm{~W}<\mathrm{y} 4)), \mathrm{W}((\mathrm{W}<\mathrm{y} 1) \&(\mathrm{~W}>\mathrm{y} 2) \&(\mathrm{~W}>\mathrm{y} 3) \&(\mathrm{~W}<\mathrm{y} 4)$ )];

16. Plotting the optimum zone chart without PCA

a. Plot original RPM and WOB data points

figure()

plot(RPM,WOB,'g.');

title('Classification based on Downhole Data');

ylabel('Weight on Bit Klb');

xlabel('Rotary Speed RPM');

hold on

b. Classify the data points based on results of downhole vibration measurements

Torsional=data $(:, 7)>50$;

plot(data(Torsional,1), data(Torsional,3),'r.');

Lateral $=\operatorname{data}(:, 9)>2.5$;

plot(data(Lateral,1),data(Lateral,3),'k.');

legend ('GoodDrilling', 'Torsional', 'Lateral') 
c. Connect the upper and lower limits of WOB and RPM

$\mathrm{Y}=[\mathrm{R} P ; \mathrm{R} \mathrm{Q} ; \mathrm{S} \mathrm{Q} ; \mathrm{S} \mathrm{P} ; \mathrm{R} \mathrm{P}]$;

$\operatorname{plot}\left(\mathrm{Y}(:, 1), \mathrm{Y}(:, 2),{ }^{\prime}\right.$ ','linewidth', 3);

axis([150 18018 42])

hold off;

17. Plotting the optimum zone chart and the safe zone chart with PCA

a. Plot generated RPM and WOB data points after inverse PCA has been done figure()

plot (N,W,'g.');

title('Classification with PCA and Safety');

ylabel('Weight on Bit Klb');

xlabel('Rotary Speed RPM')

b. Connect the upper and lower limits of the newly generated RPM and WOB

$\mathrm{Y}=[\mathrm{Ra} \mathrm{Pa} ; \mathrm{Ra} \mathrm{Qa} ; \mathrm{Sa} \mathrm{Qa} ; \mathrm{Sa} \mathrm{Pa} ; \mathrm{Ra} \mathrm{Pa}]$;

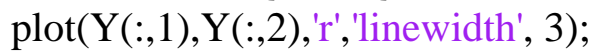

hold on;

$\mathrm{Za}=[\mathrm{Ra} \mathrm{Pa} ; \mathrm{Ra} \mathrm{Qa} ; \mathrm{Sa} \mathrm{Pa} ; \mathrm{Sa} \mathrm{Qa}]$;

plot(Za(:,1),Za(:,2),'ro','markersize', 20);

c. Connect the coordinates of the safe zone

$\mathrm{Yn}=[\mathrm{Ra} \mathrm{Pm} ; \mathrm{Sm} \mathrm{Qn} ; \mathrm{Sn} \mathrm{Pm} ; \mathrm{Sm} \mathrm{Pn} ; \mathrm{Ra} \mathrm{Pm}]$;

$\operatorname{plot}\left(Y n(:, 1), \operatorname{Yn}(:, 2),{ }^{\prime}\right.$ ','linewidth',3);

$\mathrm{Zn}=[\mathrm{Ra}$ Pm;Sm Qn;Sn Pm;Sm Pn];

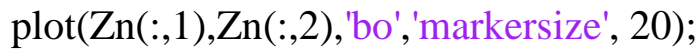

d. Locate the centroid of the safe zone using Kmeans clustering

[idx,C]=kmeans(SafeZone, 1$)$;

$\operatorname{plot}\left(\mathrm{C}(:, 1), \mathrm{C}(:, 2),{ }^{\prime} .{ }^{\prime}\right.$, 'markersize',35)

e. Classify the data points based on results of downhole vibration measurements

Torsional $=$ data $(:, 7)>50$;

plot(data(Torsional,1),data(Torsional,3),'y.');

Lateral=data $(:, 9)>2.5$;

plot(data(Lateral,1), data(Lateral,3),'k.');

axis([150 18018 42])

hold off; 


\section{APPENDIX II: CUSTOMER VALUE PROPOSITION CANVAS WITH REFERENCES}

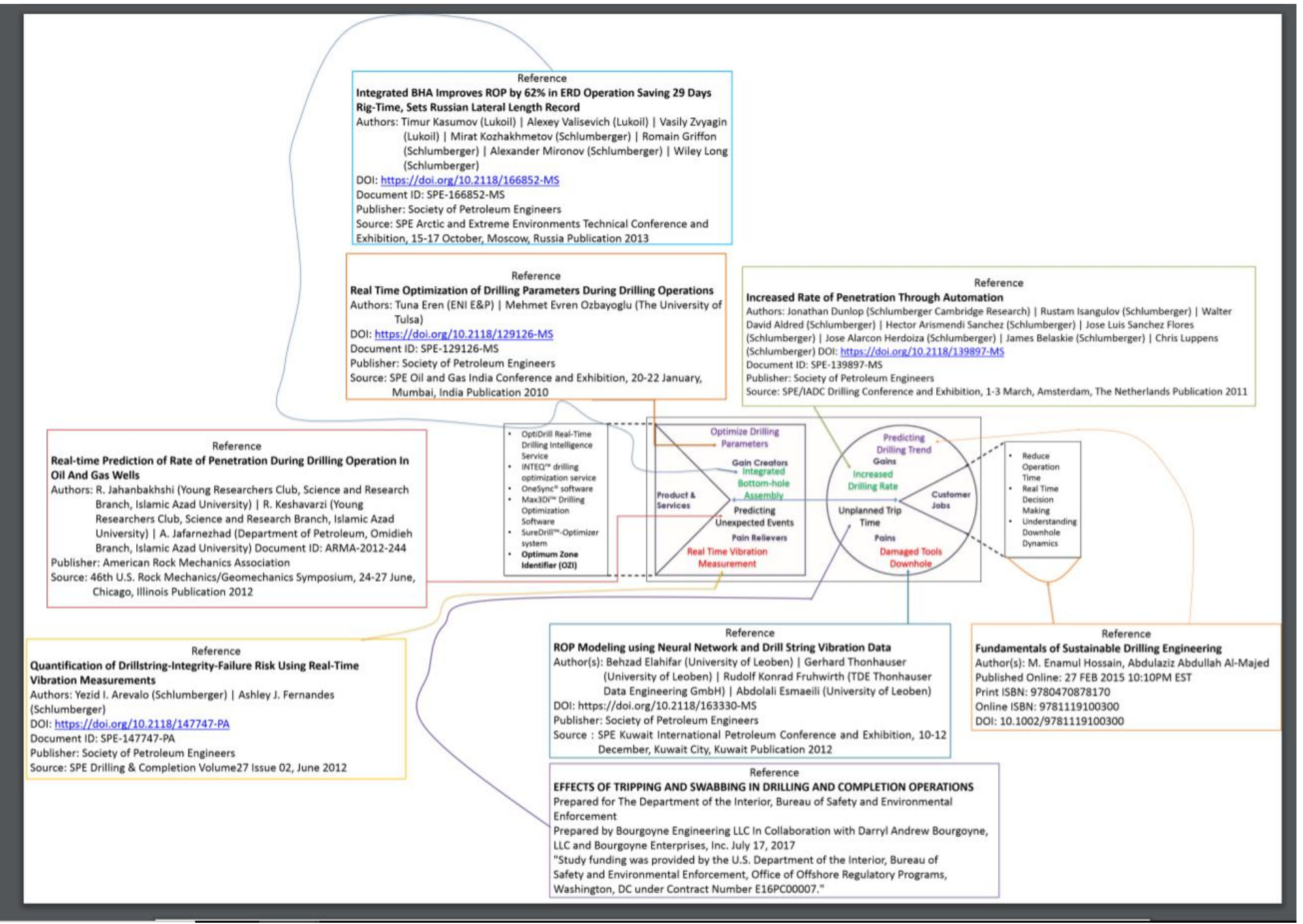

